$$
D \circ E / E R / 53241-7-P t, 3
$$

\title{
Ion Temperature Measurements in the Maryland Spheromak
}

\author{
Jean-Luc Gauvreau
}

1992

\section{DISCLAIMER}

This report was prepared as an account of work sponsored by an agency of the United States Government. Neither the United States Government nor any agency thereof, nor any of their employees, makes any warranty, express or implied, or assumes any legal liability or responsibility for the accuracy, completeness, or usefulness of any information, apparatus, product, or process disclosed, or represents that its use would not infringe privately owned rights. Reference herein to any specific commercial product, process, or service by trade name, trademark, manufacturer, or otherwise does not necessarily constitute or imply its endorsement, recommendation, or favoring by the United States Government or any agency thereof. The views and opinions of authors expressed herein do not necessarily state or reflect those of the United States Government or any agency thereof. 


\title{
Ion Temperature Measurements in the Maryland Spheromak
}

\author{
Jean-Luc Gauvreau \\ Dissertation submitted to the Faculty of the Graduate School \\ of the University of Maryland in partial fulfillment \\ of the requirement for the degree of \\ Doctor of Philosophy \\ 1992
}

Advisory Committee:

Professor George Goldenbaum, Chairman/Advisor Associate Professor Richard Ellis

Professor Hans Griem

Associate Research Scientist Parvez Guzdar

Professor Charles Striffler 


\begin{abstract}
Title of Dissertation: Ion Temperature Measurements in the Maryland Spheromak

Jean-Luc Gauvreau. Doctor of Philosophy, 1992

Dissertation directed by: Dr. George Goldenbaum, Professor of Physics Department of Physics and Astronomy

Initial spectroscopic data from MS showed evidence of ion heating as deduced from the line widths of different ion species. Detailed measurements of OIV spectral emission line profiles in space and time revealed that heating takes place at early time, before spheromak formation and is occurring within the current discharge. The measured ion temperature is several times the electron temperature and cannot be explained by classical (Spitzer) resistivity. Classically, ions are expected to have lower temperatures than the electrons and therefore, lower temperatures than observed. High ion temperatures have been observed in different RFP's and Spheromaks but are usually associated with relaxation to the Taylor state and occur in the sustainment phase. During formation, the current delivered to start the discharge is not axisymmetric and as a consequence, $\mathrm{X}$-points appear in the magnetic flux. A two dimensional analysis predicts that magnetic reconnection ocurring at an $\mathrm{X}$-point can give rise to high ion heating rates. A simple 0 -dimensional calculation showed that within the first 20 $\mu s$, a conversion of mass flow kinetic energy into ion temperature could take place due to viscosity.
\end{abstract}


Dedication

A mes parents 


\section{Acknowledgments}

For his guidance. his encouragements and his constant optimism. I would like to thank my advisor, Professor George Goldenbaum. I would like also to thank Professors DeSilva. Ellis and Griem for their suggestions and their availability. The discussions I had with Dr. John Finn and Dr. Parvez Guzdar were most helpful for this work.

In the MS group, I had the pleasure to work with Dr. Cecil Chin-Fatt who shared with us his experience of the machine and of experimental plasma physics. It was also a pleasure to work with Dr. Roger Hess who maintained excellent computer systems and who gave me good advice particularly on spectroscopy. I must thank Mr. David Miller and Mr. Ken Diller for their technical assistance all over the years and Dr. J.S. Wang and Mr. Mark Ford for their good advice about electronics. Thank you to fellow graduate students Bruce Barrow, Claude Côté, Alex Filuk, Fu-Kwun Hwang and Scott Janz for their friendship, constant cooperation and shared data. The Laboratory for Plasma Research was a very supportive environment. I enjoyed sharing physics ideas and playing music with Thi Pham during my years at Maryland.

Finally, I am grateful to my parents and my sister for their encouragements and their love all along. 


\section{Contents}

List of Tables viii

List of Figures $\quad$ ix

1 Introduction 1

1.1 Present sources of energy ................ 1

1.2 Fusion: a future source of energy ? . . . . . . . . . . . 2

1.3 Present technologies ................... 4

1.4 Introduction to Spheromaks . . . . . . . . . . . . . 6

1.5 Summary of this thesis . . . . . . . . . . . . 7

2 Description and Operation of MS 10

2.1 Brief history of MS ................ 10

2.2 The classical Spheromak . . . . . . . . . . . . . . 13

2.3 Description of MS ................. 15

2.3.1 Bias coils ................. 17

2.3.2 $I_{z}$ electrodes ................ 17 
2.3.3 Reversal coils . . . . . . . . . . . . . . . . . . . . . . 19

2.3.4 Energy storage capacitor banks . . . . . . . . . . . 20

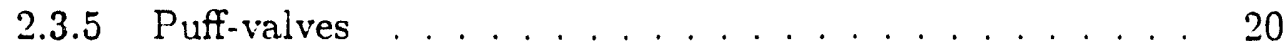

2.3.6 Figure-eight coils . . . . . . . . . . . . . . . . . . 21

2.3 .7 Pumps . . . . . . . . . . . . . . . . . . . 21

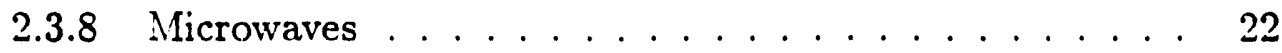

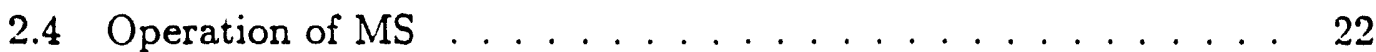

3 General Diagnostics 26

3.1 Vacuum Diagnostics . . . . . . . . . . . . . . 26

3.2 Magnetic Probes . . . . . . . . . . . . . . . . . 27

3.3 Rogowski probe . . . . . . . . . . . . . . . . . . . . . 28

3.4 High voltage probe . . . . . . . . . . . . . . . . 33

3.5 Interferometry $\ldots \ldots \ldots \ldots \ldots \ldots \ldots \ldots$

3.6 Langmuir Probe . . . . . . . . . . . . . . . 35

3.7 Bolometry . . . . . . . . . . . . . . . . . . . 36

4 Spectroscopic Diagnostics 39

4.1 Monochromators . . . . . . . . . . . . . . . . . . 39

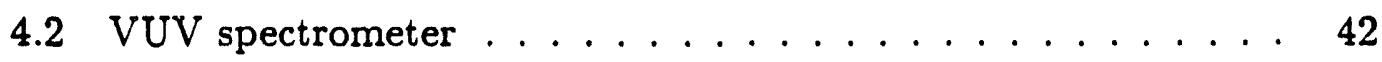

4.3 Linear OMA . . . . . . . . . . . . . . . . . . . . 44

$4.4 \quad 2-\mathrm{D}$ OMA $\ldots \ldots \ldots \ldots \ldots \ldots \ldots \ldots \ldots \ldots$

4.4 .1 Gating the OMA . . . . . . . . . . 49 
4.4 .2 Cooling the OMA . . . . . . . . . . . . 49

4.4 .3 Rotating Slit . . . . . . . . . . . . . 50

4.4.4 Wide Angle Lens System . . . . . . . . . . . 52

4.4.5 Calibration of the Optics $\ldots \ldots \ldots \ldots \ldots$

4.4.6 Relative wavelength calibration . . . . . . 57

4.5 Line Broadening Mechanisms . . . . . . . . . . . 57

5 Results $\quad 60$

5.1 General observations . . . . . . . . . . . . . . 6 60

5.2 Processing of data $\ldots \ldots \ldots \ldots \ldots \ldots$

5.3 Single shot time resolved results . . . . . . . . . . . . . 64

5.3.1 Ion temperature and line intensity . . . . . . 64

5.3 .2 Impurities $\ldots \ldots \ldots \ldots \ldots \ldots \ldots$

5.3.3 $H_{\beta}$ and $D_{\beta}$ data $\ldots \ldots \ldots \ldots \ldots 6$

5.4 Single shot space resolved results $\ldots \ldots \ldots \ldots \ldots$

5.4 .1 Inversion method $\ldots \ldots \ldots \ldots \ldots \ldots$

5.4.2 Inversion of a test function $\ldots \ldots \ldots \ldots \ldots$

5.4 .3 OIV Chord data $\ldots \ldots \ldots \ldots \ldots \ldots \ldots$

5.4.4 Results of the inversion $\ldots \ldots \ldots \ldots \ldots$

5.4 .5 Plasma symmetry $\ldots \ldots \ldots \ldots \ldots$

6 Discussion and Conclusion $\quad 88$ 
6.1 Helicity transport and anomalous ion heating .................... 92

6.1.1 Results in RPF's . . . . . . . . . . . . . . 92

6.1.2 Energy and helicity global balance . . . . . . . . . . 93

6.1.3 Modified Ohm's law ............... 95

6.2 Tearing modes . . . . . . . . . . . . . . . 96

$6.3 \mathrm{X}$-point heating .................. 101

6.3.1 Heating estimates .............. 103

6.4 Energy balance . . . . . . . . . . . . . . . 108

6.5 Conclusion ............................. 11

$\begin{array}{lr}\text { Appendices } & 113\end{array}$

$\begin{array}{lr}\text { A High voltage pulse circuits. } & 113\end{array}$

B Ion heating from fluctuations $\quad 115$

$\begin{array}{lr}\text { References } & 118\end{array}$ 


\section{List of Tables}

1.1 Comparison between the tokamak, the RFP and the spheromak machines. . . . . . . . . . . . . . . . . 7

6.1 Thermal equilibration times between electrons, $D^{+}$and OIV. . 106 


\section{List of Figures}

2.1 Poloidal flux contours of the classical spheromak and its magnetic field profiles on the midplane. . . . . . . . . . . . 16

2.2 MS vacuum vessel. Top view. . . . . . . . . . . . . . . 18

2.3 MS formation scheme. . . . . . . . . . . . . . . . . . . . 24

3.1 Typical toroidal and poloidal magnetic trace in the midplane at various radii . . . . . . . . . . . . . . . . 29

3.2 Midplane $\mathrm{B}_{\phi}$ fields at $\mathrm{t}=(0.25,0.35) \mathrm{msec}$, show that the plasma was forming symmetrically on axis, and remained centered after shielding the reversal coil from the plasma. . . . . . . . . . .

3.3 Poloidal field structure on the R-Z plane for with glass plate barriers. . . . . . . . . . . . . . . 31

3.4 Typical traces of the reversal current, $I_{z}$ current and $I_{z}$ voltage

3.5 High voltage probe circuit diagram. . . . . . . . . 33

3.6 Line average electron densities at different chord parameters with glass plate barriers. . . . . . . . . . . . 35

3.7 Radiation power density integrated over time. . . . . . . . . 37

3.8 Pyroelectric signal as a function of chord parameter for different times. . . . . . . . . . . . . . . . . 
4.1 Line of sights used to monitor CIII and CIV impurity lines with the monochromators . . . . . . . . . . . . . . 40

4.2 CIII and CIV signals under clean and dirty conditions. . . . . . 41

4.3 Optical set-up to scan the chamber. . . . . . . . . . 42

4.4 Radial behavior of the electron temperature obtained from CIV to CIII line ratio. . . . . . . . . . . . . . . . . . . . 43

4.5 Impurity profiles obtained with the linear OMA. . . . . . 45

4.6 OMA head $\ldots \ldots \ldots \ldots \ldots \ldots \ldots$

4.7 Diagram of the OMA mounted on a $1 \mathrm{~m}$ spectrometer. . . . . 48

4.8 Wide angle lens optical system designed from ray tracing and actual system used . . . . . . . . . . . . . 54

4.9 Calibration of the wide lens for an object placed at 1 meter. . . 55

5.1 Ion temperature of OII, OIII and OIV as a function of time. . 62

5.2 Typical fit of an OIV experimental line shape to a Gaussian plus constant slope background. . . . . . . . . . . 63

5.3 Ion temperature and intensity as a function of time for CIII, CIV and OIV measured using a rotating slit. . . . . . . . 65

5.4 Spectrum of impurities as a function of wavelength and time from 2200 to $5400 \AA \ldots \ldots$. . . . . . . . . . . 67

5.5 $H_{\beta}$ and $D_{\beta}$ spectra for different chords and times before performing gettering $\ldots \ldots \ldots \ldots \ldots \ldots$

5.6 $H_{\beta}$ and $D_{\beta}$ spectra for different chords and times after performing gettering $\ldots \ldots \ldots \ldots \ldots \ldots \ldots$

5.7 Diagrams showing how chords intercept different radii for two equivalent geometries. . . . . . . . . . . . . . 
5.8 Temperature and intensity radial profiles used to test the inversion routine and their line of sight chord profiles . . . . . i5

5.9 Simulated temperature and intensity chord data added with noise and their inversions . . . . . . . . . . 76

5.10 Typical inverted profile and its fit to a Gaussian. . . . . . . 78

5.11 Intensity and temperature measurements and their inversion for OIV $(3063 \AA)$ at $30 \mu s \ldots \ldots \ldots$. . . . . . . . . . .

5.12 Intensity and temperature measurements and their inversion for OIV $(3063 \AA)$ at $50 \mu s \ldots \ldots \ldots \ldots . \ldots \ldots$

5.13 Intensity and temperature measurements and their inversion for OIV $(3063 \AA)$ at $70 \mu \mathrm{s} \ldots \ldots \ldots \ldots \ldots$

5.14 Intensity and temperature measurements and their inversion for OIV $(3063 \AA)$ at $110 \mu \mathrm{s} \ldots \ldots \ldots$. . . . . . . . .

5.15 Intensity and temperature measurements and their inversion for OIV $(3063 \AA)$ at $160 \mu \mathrm{s} \ldots \ldots \ldots \ldots$. . . . . . . .

5.16 Picture of the plasma discharge taken with a pin-hole camera. End view.

5.17 Reference field of view and contour of constant intensity of the plasma discharge at $950 \mu s$. End view. . . ...................

5.18 Contour of constant intensity of the plasma discharge taken with the OMA at 1000 and $1100 \mu s$. End view. .

6.1 Current density as a function of radius in the midplane. With glass plate barriers. . . . . . . . . . . . . .

6.2 Contours of constant $r-\phi$ flux in the midplane for conditions without glass plate barriers. . . . . . . . . . . . . .

6.3 Radial profile of the safety factor $q$ in the midplane for conditions without glass plate barriers. . . . . . . . . . 
6.4 Simulation contour of constant flux for an $m=4$ island mode with and without reverse current . . . . . . . . . . . . 102

6.5 Simulation of X-point heating for MS parameters . . . . . 107

6.6 Total power input through the electrode during formation . . 110

A.1 High voltage circuits used to gate the OMA. . . . . . . . . 114 


\section{Chapter 1}

\section{Introduction}

\subsection{Present sources of energy}

Our needs for energy is constantly growing from year to year. Fossil fuels, the main source of energy at present are expected to be slowly phased out either because of exhaustion of primary material or because of the pollution brought by their use. The former seems to be our main concern at this moment. Soft energies like solar and eolian energies appear to be either too inefficient or too costly to replace energy production based on oil and coal. Where available, hydro-electricity seems to adequately provide great quantities of energy but sometimes at the cost of destroying its surrounding natural environment. Fission is used in many countries but its use is controversial because of the radioactive products associated with it. 
Fission involves a heavy nucleus split by a neutron into fast fragments releasing energy in the process. i.e..

$$
{ }_{92} \mathrm{U}+n \rightarrow{ }_{92} \mathrm{U}^{*} \rightarrow{ }_{56} \mathrm{Ba}+{ }_{36} \mathrm{Ir}+\text { Energy }
$$

Although it has the potential of producing energy for the next several hundred years, it has its problems. The output product is radioactive and typically has a half-life decay of 10,000 years. Handling and storage of the contaminants is therefore a problem for present and future generations. In case of a malfunction. uncontrolled fission reaction can take place with the main consequence being the release of a large quantity of radioactive material in the atmosphere and could be a threat to the population and environment.

\subsection{Fusion: a future source of energy ?}

A cleaner energy source would be available, if we could recreate conditions similar to what exists inside the sun, within our laboratory. Fusion is the power source of stars. In the first burning stage of a star, four light hydrogen nuclei combine to create helium and in the process release energy.

$$
4 H \rightarrow H e+2 e^{+}+2 \nu+\text { Energy }
$$

In an actual laboratory, the fusion of deuterium and tritium would be an 
easier reaction to perform.

$$
D+T \rightarrow H e+n+\text { Energy }
$$

Although tritium is a rare element in nature, it can be bred from lithium as explained in the next section.

This nuclear reaction releases a non radioactive, non-toxic secondary product: helium. Very likely, it would make its way to the higher altitudes and would eventually leave the planet. Even if done on a large scale, no effects on the humans, plants, ozone layer, or on the global warming are expected from this helium gas. Fusion differs from fission in that, excluding the neutron irradiated wall materials, no actinide wastes nor the equivalent of spent fuel has to be disposed of.

The primary fuels for D-T fusion are deuterium and lithium. Lithium is used as a blanket to convert the fast neutrons into thermal energy and in the process it breeds the necessary tritium for the fusion reaction. Deuterium and tritium are so abundant in nature that D-T fusion could be an inexhaustible source of energy. Deuterium is present in sea water at a concentration of 1 deuterium atom per 6700 hydrogen atom. Lithium is present in the earth's crust at $30 \mathrm{ppm}$ by weight and $.2 \mathrm{ppm}$ in the ocean. To produce one gigawatt of energy for one year would consume between .5 and 5 tons of fuel. We have enough lithium on land to supply D-T reactors for several hundred years. An other possibility is to get it from the moon.

A lot of efforts in research are still necessary in order to harness this energy 
in a controlled manner.

\subsection{Present technologies}

In a star, the reacting nuclei are brought together and maintained at high density and temperature due to the star's own gravitational field. In a laboratory a different means will have to be used to achieve temperatures sufficient for fusion and to keep the nuclei in a finite volume. The main approaches to fusion are inertial and magnetic confinement. In all those cases, plasmas are involved. A plasma is a gas at a temperature high enough that all the atoms get separated into ions and electrons. It is an ionized gas. Unlike an unionized gas, it can interact strongly with a magnetic field.

Break-even is achieved when the energy carried by the neutrons equals the energy input in the reactor. Ignition is achieved when the energy carried by the alpha particles (helium nucleus) is sufficient to maintain the nuclear reaction by reheating the reacting nuclei.

\section{- Inertial confinement:}

The goal of inertial confinement is to uniformly irradiate the surface of a solid fuel pellet with high energy beams of light or particles. Compressed to very high densities ( $\sim 10^{3}$ solid densities) and heated locally to the temperature required for ignition, fusion power could be obtained before the pellet disperses itself due to the increasing pressures.

- Magnetic confinement: 
In magnetic confinement, a plasma is created in a chamber and is heated either by electric currents, the injection of energetic particles or electromagnetic waves. Magnetic fields are used to keep the hou plasma confined and away from the walls.

Each $\mathrm{D}-\mathrm{T}$ reaction produces a $3.5 \mathrm{MeV}$ alpha particle, which can slow down in the plasma and release its energy, and a $14 \mathrm{MeV}$ neutron which escapes from the plasma. Neutrons can be slowed down and their energy recovered but in the process they also activate nuclei in the walls. By choosing the proper materials to build the reactor, the components would remain radioactive for only a limited number of years. Reactor design would have been simpler if only radiation was escaping from the plasma, heating a surrounding blanket by absorption. On the other hand, the high energy neutrons can be used for breeding tritium fuel from interaction with a lithium blanket within the wall of the reactor. Tritium is radioactive however, and has a half-life of 12.3 years. Critical accidents are impossible because the reaction chamber contains just enough fuel to sustain a thermonuclear burn for 10 seconds.

The most promising magnetic confinement machine is the tokamak. Tokamaks have been studied since the early '50's, when Andrei Sakharov and a team of researchers at the Kurchutov Institute in the Soviet Union invented it [1]. In Russian, it is an acronym for Toroidal Magnetic Chamber. Being in the shape of a donut, it has a major radius ranging from less than $40 \mathrm{~cm}$ in the smaller tokamaks to 3 meters in large tokamaks like TFTR and JET. ITER, a device similar to the tokamak is under conceptual design to achieve controlled ignition and will have a major radius of $6 \mathrm{~m}$ [3]. For fusion break- 
even to occur. the required conditions are a temperature of at least $10.000 \mathrm{eV}$ and a confinement time-density product greater than $10^{14} \mathrm{sec} / \mathrm{cm}^{3}$ (Lawson criteria). For reasonable power output per unit volume, a density of $10^{14} \mathrm{~cm}^{-3}$ is required and this implies that the plasma must last at least 1 second. The required $10,000 \mathrm{eV}$ for fusion have been achieved in large tokamaks $[4,5]$. A near break-even condition was observed on the JET tokamak in 1991 when they operated with D-T fuel for the first time. An experimental tokamak is built at a cost of several hundred million dollars and requires a team of several hundred researchers, engineers and technicians to operate. A tokamak reaitor could cost up to 10 times more. Because of the high cost associated with Tokamaks, several alternate reactor concepts were investigated. The Stellarator, Reversed Field Pinch (RFP), Field Reversed Configuration (FRC) and Spheromak are examples of alternate concept confinement schemes.

\subsection{Introduction to Spheromaks}

In Table 1.1 we show a comparison of some features of the tokamak, the RFP and the spheromak. The RFP was included because it has similarities with both machines.

Spheromaks are much less developed than the other types of confinement devices. On the other hand, because they are more compact, it is expected that the same magnitude of currents and magnetic fields might be achieved at a lower cost. At the present time, the life-time of a spheromak plasma is in the order of milliseconds compared to about 1 second for RFP's and tens of 


\begin{tabular}{|l|l|}
\hline & Tokamak \\
\hline $\begin{array}{l}\text { Main current } \\
\text { Formation } \\
\text { Sustainment } \\
\text { Machine }\end{array}$ & $\begin{array}{l}\text { Toroidal } \\
\text { Inductive toroidal drive } \\
\text { Inductively maintained, non-Taylor state } \\
\text { Magnetic core. inner wall (Toroid) }\end{array}$ \\
\hline & RFP \\
\hline $\begin{array}{l}\text { Main current } \\
\text { Formation } \\
\text { Sustainment } \\
\text { Machine }\end{array}$ & $\begin{array}{l}\text { Toroidal } \\
\text { Inductive toroidal drive } \\
\text { Inductively maintained, Taylor state } \\
\text { Magnetic core, inner wall (Toroid) }\end{array}$ \\
\hline $\begin{array}{l}\text { Main current } \\
\text { Formation }\end{array}$ & $\begin{array}{l}\text { Toroidal and poloidal currents are about equal } \\
\text { Inductive toroidal drive, } \\
\text { Inductive or non-inductive poloidal drive }\end{array}$ \\
$\begin{array}{l}\text { Sustainment } \\
\text { Machine }\end{array}$ & $\begin{array}{l}\text { Self-maintained, Taylor state } \\
\text { No magnetic core, no inner wall (Spheroid) }\end{array}$ \\
\hline
\end{tabular}

Table 1.1: Comparison between the tokamak, the RFP and the spheromak machines.

seconds for tokamaks. Typical electron temperatures for a spheromak is 10$100 \mathrm{eV}$ while in a tokamak it can reach 2 orders of magnitude higher. Much work on the spheromak concept is needed to bring it to the level necessary where a decision on its value as a reactor can be made.

\subsection{Summary of this thesis}

This thesis concentrates on describing the observations of broad emission line profiles and explaining them as anomalously high ion temperatures. The high 
temperatures involve a non-classical heating effect and is not a phenomenon unique to MS. Several other spheromak $[6,7]$ and RFP $[8,9]$ experiments have reported such observations. The usual explanation is that by some process. magnetic energy is transferred to the ions through fluctuations. A major difference is that in MS the highest ion temperatures are observed at early times during formation, while in other machines it is observed during the sustainment phase or the decay.

As the vacuum vessel was modified on a continuing basis over the years. spectral line observations were most helpful in identifying the impurities present. telling us if conditions were improving. High ion temperatures were also observed during the various stages of modification. The observation of a wide oxygen line next to a narrow heavy impurity line like chromium convinced us that the heating might be real and is not just due to an unexplained large instrumental width. The question also arises about whether it is an actual temperature or just the superposition of different directed motions. When looking along the axis, sometimes non-Gaussian wavelength profiles were observed, implying mass motion of the plasma. In the present study a detailed measurement of OIV ion emissions as a function of space and time in the mid-plane is attempted. The line shapes are mostly Gaussian, and they are almost always centered on the unshifted wavelength. This suggests that if different cells of directed motions are present, they occur on such a small spatial scale that it is effectively a temperature or will become a temperature through collisions or viscosity.

When combining spectroscopic data with magnetic data, one tentative explanation for ion heating will be that initial mass flow motions are transformed 
into temperature through viscosity. Tearing modes and helicity transport will also be considered. Because high energy ions is what is ultimately needed to obtain fusion, identifying the different heating mechanisms is an important task. 


\section{Chapter 2}

\section{Description and Operation of}

\section{MS}

\subsection{Brief history of MS}

MS is a fourth generation spheromak at the University of Maryland. As does its predecessors PS-1 [13], PS-2 [18], PS-3 and PS-3.5 [19,20], it uses a combination of theta and $\mathrm{Z}$ discharges. Other spheromaks elsewhere have been produced with different methods: flux core Electrodeless Induction in S-1 [14], the Magnetized Coaxial Gun [15] and the kinked Z-pinch in CTX [16]. Early spheromaks were radiation dominated and did not exceed electron temperatures of $10 \mathrm{eV}$ to $20 \mathrm{eV}$ [17]. MS was initially designed to have sufficient heating that the radiation barrier could be exceeded. The MS Spheromak has 
a formation time of $100 \mu s$ and a plasma lifetime of $400 \mu s$. These parameters are an order of magnitude larger than for the previous experiment PS-3.5.

Operation of the Maryland Spheromak began in 1986. Initial work concentrated on identifying the cause of a plasma shift observed with the magnetic probes [21]. Different stabilization methods were used: a cylindrical core, cylindrical liner, cone or funnel shaped conductors on axis [23] and finally figure-eight coils [11]. The latter had the most influence on the plasma stability. However a shift was still observed repeatedly in the same direction. Thanks to a visitor from the University of Tokyo, Dr Yasushi Ono, the cause was found to be the interaction of the plasma with the bare reversal coil. Wrapping the coil with a rubber based tape covered with fiberglass showed that the shift could be eliminated, but introduced many impurities. After trying several insulating materials, teflon tape was used to electrically insulate the coil and a thin (.001") nichrome tape covered the teflon so that only metal is exposed to the plasma.

Having stopped the equilibrium shift, the emphasis changed to increasing the temperature and hence the plasma lifetime. The limiting factor was felt to be radiation from impurities. Because visible marks of erosion appear on the electrodes after several shots, it was suspected that they could contribute to the impurity content of the plasma. Several types of electrode tips were used. Aluminum was found to be a too soft metal. Elkonite, which is a hard alloy made of copper and tungsten was found to be superior to stainless steel for its resistance to erosion. Copper and tungsten did not appear as being the major impurity radiations from the plasma. The main impurities were found spectroscopically to be oxygen, carbon and aluminum. It is suspected 
that currents flowing backward on the alumina (Aluminum oxide) electrode insulators to the walls scrape off aluminum and oxygen. Attempts to stop this current were unsuccessful. Carbon could come from the teflon tape and also from pump oils.

Finally, with the magnetic diagnostic [21], it was found that approximately half of the $I_{z}$ current follows field lines around the outside of the reversal coils near the wall of the vessel instead of flowing near the machine axis. Polyethylene barriers were installed to reduce the outside current and showed a dramatic increase in current on axis and in its associated toroidal field. Because they contributed also to an increased electron density presumably from impurities, they were replaced with glass plates. The glass plates blocked completely the unwanted current path and did not introduce a large amount of impurities.

Elkonite electrode tips, teflon/nichrome reversal coil wrapping and glass plates are the standard conditions in which data are taken in the present thesis.

Further work concentrated in creating a hotter and longer lived plasma, mostly by impurity control methods since it appeared to be radiation dominated [22]. 


\subsection{The classical Spheromak}

The persistence of magnetic fields observed in the Crab Nebula [24], led Woltjer to suggests that the magnetic structure is force-free [25],

$$
\mathrm{J} \times \mathrm{B}=0
$$

meaning that $\mathbf{J}$ is parallel to $\mathbf{B}$ in every point. In the process of demonstrating this effect mathematically, he discovered a new conserved quantity in ideal MHD: the magnetic helicity,

$$
H_{\psi}=\frac{1}{2 \mu_{0}} \int_{v} \mathbf{A} \cdot \mathbf{B} d^{3} x
$$

The integration is over a flux tube and helicity is conserved within a flux tube for an infinitely conducting plasma. It is a measure of the twisting and linking of magnetic fluxes. Then by minimizing the magnetic energy

$$
W=\frac{1}{2 \mu_{0}} \int_{v} B^{2} d^{3} x
$$

subject to the constraint that helicity is conserved locally, Woltjer obtained a force-free solution,

$$
\nabla \times \mathbf{B}=\mu \mathbf{B},
$$

where $\mu$ depends on the flux tube. This magnetic state is achieved if a mechanism exists to dissipate some magnetic energy from the initial configuration. This final state is highly dependent on the initial state.

Taylor applied this idea to RFP's to explain their relaxation to some stable 
state regardless of their initial conditions. It was observed that under certain conditions, the toroidal magnetic field is reversed in the outer radius for this stable state [26]. In this case, because the small resistivity of the plasma is taken into account, the helicity is assumed to be conserved globally instead of locally. When minimizing the magnetic energy subject to constant total magnetic helicity, Taylor found that a force free state is achieved [27],

$$
\mu_{0} \mathbf{J}=\nabla \times \mathbf{B}=\mu \mathbf{B},
$$

where $\mu$ is now independent of space, it depends only on boundary conditions. The final Taylor state is obtained independently of the formation scheme and initial conditions.

The solution which in cylindrical geometry is independent of $z$ or $\phi$, is given by Bessel functions of the zero and first order:

$$
\begin{aligned}
& B_{z}=B_{0}(k r) \\
& B_{\phi}=B_{0} J_{1}(k r) \\
& B_{r}=0
\end{aligned}
$$

It is independent of $z$ or $\phi$. This solution also exhibits the reversal of toroidal field observed in RFP's. It occurs when $k r$ is larger than the first zero of the $J_{0}$ bessel function.

For the MS spheromak, where major radius and the axial length are of the 
same order, it is more appropriate to solve this equation in spherical geometry. The explicit solution for the classical spheromak is [28],

$$
\left.\begin{array}{ll}
B_{r}=3 B_{c} \cos \theta \frac{j_{1}(k r)}{k r} \\
B_{\theta}=-1.5 B_{c} \sin \theta\left(j_{0}(k r)-\frac{j_{1}(k r}{k r}\right) \\
B_{\phi}=1.5 B_{c} \sin \theta j_{1}(k r)
\end{array}\right\} \text { if } r<R
$$

Where $B_{c}$ is the magnetic field strength at the center of the spheromak, $\mathrm{k}$ is given by the boundary conditions and $\mathrm{R}$ is the radius of the separatrix which is where the toroidal field vanishes such that $J_{1}(\mathrm{kR})=0$. Plasma currents are present only inside the region defined by the separatrix. The external field consists of a uniform magnetic field plus a dipole magnetic field reated by the plasma current inside the separatrix. (See Fig. 2.1.) The "uniform field" in the MS experiment is the mirror field supplied by the bias coil. the MS experiment.

\subsection{Description of MS}

The Maryland spheromak consists of three cylindrical nonmagnetic, stainless steel chambers. The main chamber has $40 "$ diameter, $3 / 4$ " thick walls and $30 "$ length. At each end there is a cylindrical expansion chamber of $18^{\prime \prime}$ diameter 


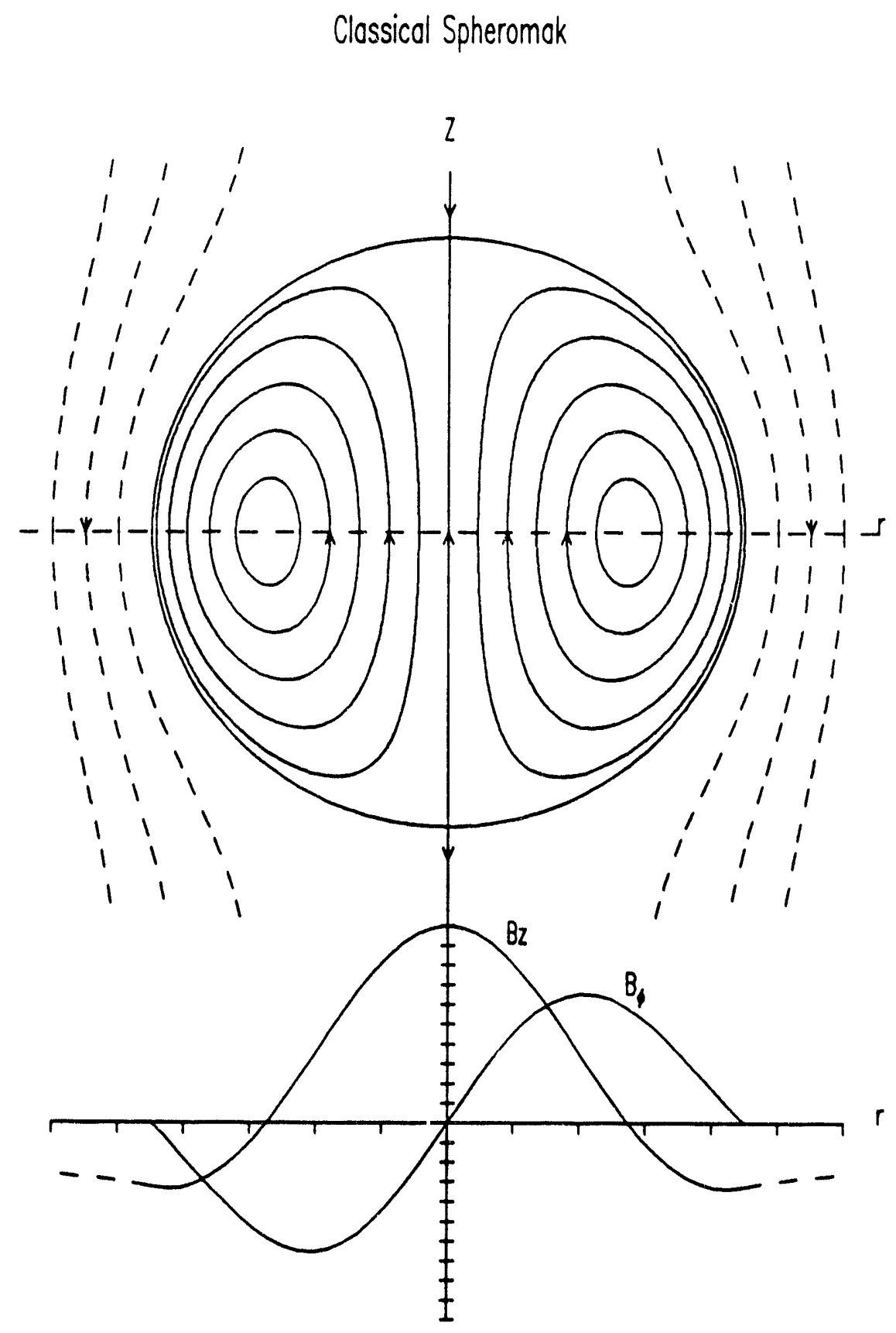

Figure 2.1: Poloidal flux contours of the classical spheromak and its magnetic field profiles on the midplane. 
and 24" length. The expansion chambers are surrounded by the bias coils, which will give a 7 second mirror field. Two reversal coils are located inside the vessel. At each end of the main chamber, there is a ring of 16 electrodes. See Fig. 2.2. Different ports allow us to probe the plasma either passively (by sending and/or receiving light) or actively (by inserting a probe in the vessel). More on diagnostics in the next chapter.

\subsubsection{Birs coils}

Each bias coil is made of 600 turns of aiuminum foil. Aluminum was used rather than copper in order to minimize the size and weight of the coils. They are activated by a power supply that can deliver up to $4.2 \mathrm{kA}$ for up to 10 seconds. A maximum field of $9 \mathrm{kG}$ could be produced at the center of the vessel. In the experimental run reported here, they are energized a few seconds before the discharge with a maximum current of $500 \mathrm{~A}$. To keep the resistivity low, the aluminum coils are cooled with liquid nitrogen.

\subsection{2 $I_{z}$ electrodes}

At each end of the sessel there is an annular ring of 16 electrodes. The electrode tips are at a radius of $25 \mathrm{~cm}$ and are pointing at an angle of $15^{\circ}$ with the horizontal toward the machine axis. Various materials were used for the replaceable electrode tips, including aluminum, steel and a low erosion copper-tungsten alloy (Elkonite). The base is made of a 1.5" diameter, 10" 


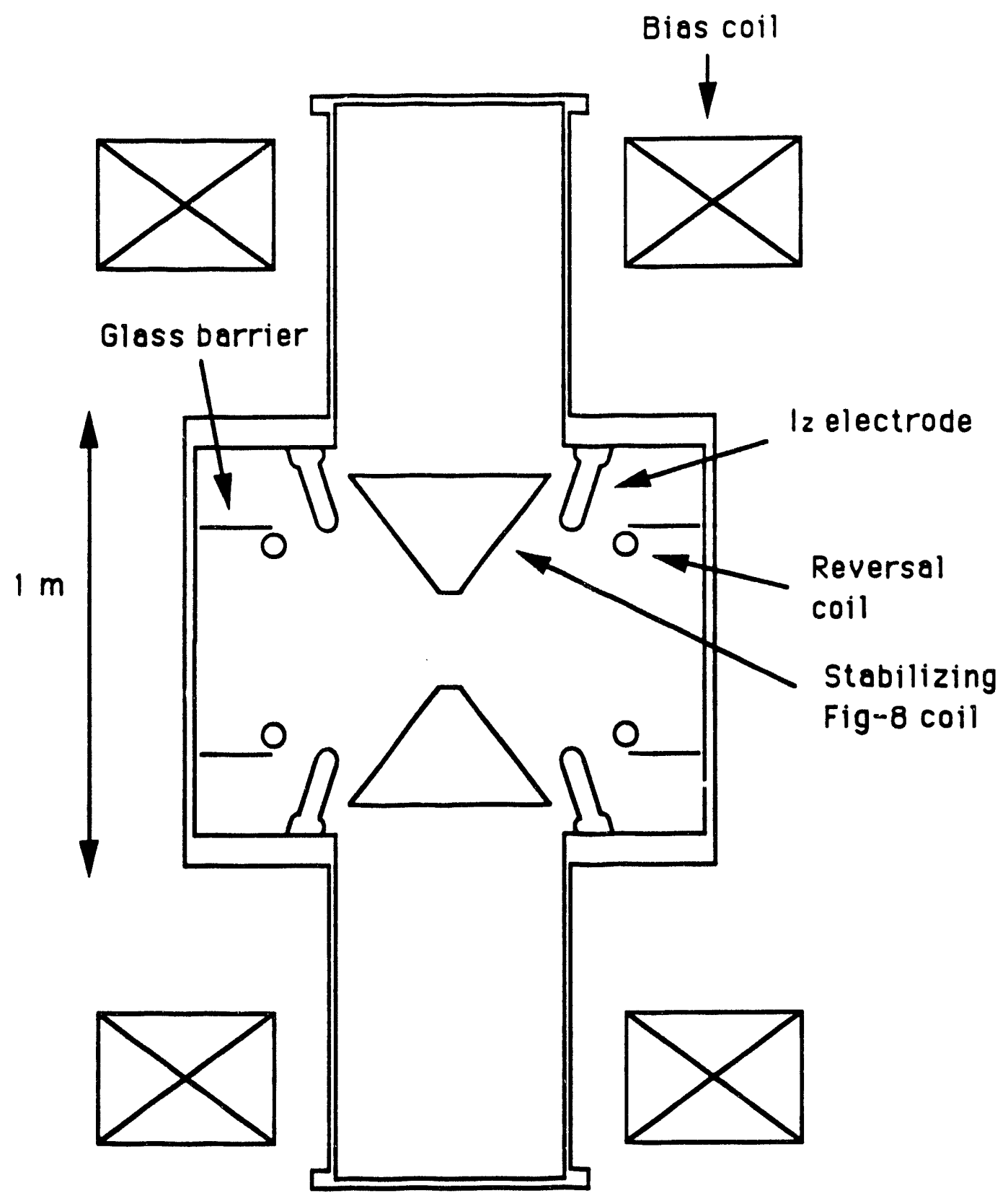

Figure 2.2: MS vacuum vessel. Top view. 
long stainless steel rod. Typically, before breakdown a voltage of $12 \mathrm{kV}$ appears between the two sets of electrodes. A peak current of almost a million Amperes can be delivered to the plasma giving an average current density of about 2 $M A / m^{2}$. An insulator jacket of alumina around each electrode was added to prevent current from flowing backward to the wall. Despite this precaution, a fraction of the current still flows back. The fraction increases with time as the insulator jackets get coated with carbon and possibly metallic deposits. The vacuum vessel itself acts as a return path for the $I_{z}$ current, resulting in a low inductance circuit. Inductors were added in series with the circuit to increase its $L / R$ time.

\subsubsection{Reversal coils}

The two reversal coils are located inside the vacuum vessel. Each coil is a one turn stainless steel toroid with a 1" minor radius and a 14" major radius. At their entrance point in the vessel they are epoxied inside a restraining yoke. Cross-bars between the coils made of alumina, prevent the coils from bending as they are fired. One should note that because they only have one turn, a small up down asymmetry due to the coils input leads exists in the machine. As explained in section 2.1, each coil is wrapped with teflon tape and nichrome foil. Typically, a peak current of $300 \mathrm{kA}$ is delivered to each coil. 


\subsubsection{Energy storage capacitor banks}

The $I_{z}$ and reversal circuits have capacitor banks as their source of current. 6 modules of 16 capacitors $(20 \mathrm{kV}, 60 \mu F)$ for the $I_{z}$ and 14 modules of 10 capacitors $(11 \mathrm{kV}, 175 \mu F)$ for the reversal. The $I_{z}$ bank is charged from a Maxwell power supply at $300 \mathrm{~mA}$ current for about 5 minutes to obtain $12 \mathrm{kV}$. The reversal bank is charged at $600 \mathrm{~mA}$ for about $2 \frac{1}{2}$ minutes to obtain $4 \mathrm{kV}$. When using all the modules, typically $200 \mathrm{~kJ}$ are stored in the reversal bank and $400 \mathrm{~kJ}$ in the $I_{z}$ bank. Charging resistances are added in the circuits to protect the power supplies. When the banks have reached their desired voltage and are ready to fire, ignitron switches are triggered, closing the circuits. A crowbar is fired while the current is decreasing and consists of an ignitron in parallel with the capacitor bank. Its purpose is to prevent the current from ringing in the machine.

\subsubsection{Puff-valves}

A single puff-valve on the center of one end-plate is used to inject the gas prior to the discharge. The valve is held shut by a spring and opened by the interaction of a solenoid current and an induced current in the valve. When a capacitor is discharged, the solenoid current and the induced current rise and the valve opens, allowing a small amount of gas to be puffed in the machine. The amount of gas injected was reproducible and was adjusted by tightening the spring. The usual gas is $D_{2}$ and less frequently $\mathrm{He}$. In static fill, the pressure obtained was 3.5 milliTorr. However, for a regular shot, the gas is 
puffed about 1 millisecond prior to the discharge, just enough time to fill the central region of the machine but not enough to become static. The reason for this method is to avoid the presence of gas and current paths near the walls. This also gives the advantage of being able to pump while puffing gas and firing. The remnant gas is therefore pumped out faster.

\subsubsection{Figure-eight coils}

Two perpendicular figure-eight coils on each ends are used to stabilize the plasma against $\mathrm{n}=1$ toroidal mode shifting and tilting instabilities [11]. A figure-eight coil has the property of maintaining the difference of flux between the two loops constant. On the other hand, the figure-eight coil does not react to a changing axisymmetric field as created by the reversal and bias coils. In MS, the coils were given an almost conical shape.

\subsubsection{Pumps}

Precautions taken to keep the vessel clean included the use of rubber gloves to handle internal parts, the use of dry nitrogen to bring the vessel to atmospheric pressure jn order to minimize the amount of water vapor coating the walls. Before closing the vessel, all the parts and surfaces that were handled are wiped with acetone. The use of materials that will outgas under vacuum, such as epoxies, plastics, vacuum grease is minimized. 
A vacuum of $10^{-7}$ Torr is achieved using mechanical pumps, turbopumps and a cryo-pump. Additional improvements to the vacuum were obtained through discharge cleaning and titanium gettering. Because discharge cleaning and gettering were time consuming and had very little effects on the plasma temperature or on the intensity of the observed impurities we discontinued their use.

\subsubsection{Microwaves}

When operating the machine at pressures below 5 millitorrs, break-down problems occurred. Jitter times of several microseconds were observed. The solution is to pre-ionize the gas through electron-cyclotron resonance such that when a high voltage is applied to the electrodes, breakdown takes place in a smooth and reproducible manner. An $8.5 \mathrm{GHz}$ magnetron was used to deliver a $200 \mathrm{~kW}$ pulse for $40 \mu \mathrm{s}$ through a wave guide terminated in a horn antenna. It was found empirically that best breakdown is achieved if the microwaves are sent at the same time as the reversal coils are fired. It appears that both contribute to plasma breakdown.

\subsection{Operation of MS}

Figure 2.3 shows the time behavior of the different currents. First, the bias field is turned on. Because of its long duration, it has time to penetrate the 
walls of the vessel and effectively provides a constant field on the time scale of the experiment. After the gas is puffed. the reversal coils are fired. reversing the magnetic field at the center (machine axis). At that point, only poloidal fields are present and there is a boundary inside of which the field lines are closed inside the vessel. This is the separatrix.

In the next step, the $I_{z}$ electrodes are fired. The current approximately flows along field lines connecting one set of electrodes to the other. This current creates the toroidal component of field.

When the sources of current to the electrodes and to the reversal coils are turned off, the plasma, by inductive effect will try to preserve its topology of toroidal and poloidal fields. Some reconnections take place and and if gross MHD instabilities do not grow to large amplitudes, the magnetic field configuration gradually reaches a force-free minimum energy state [12], meaning that all $\mathbf{J} \times \mathbf{B}$ forces are absent. From there, the plasma decays while the radius of the separatrix shrinks until it is too small to be stabilized. At that point, the spheromak dies very quickly. A shot in MS can be divided in 3 steps:

- Charging the energy banks. We use high density capacitors (typically 10 $\mathrm{kJ} / \mathrm{cap}$ ) as energy storage banks. The banks are charged over a period of several minutes and then discharged in a few hundred microseconds.

- Firing. When all the banks have attained their proper voltages, then the discharge takes place. The digitizers and all the switches controlling the discharge are triggered in the proper sequence.

- Data acquisition. Data are collected from the different diagnostics and 

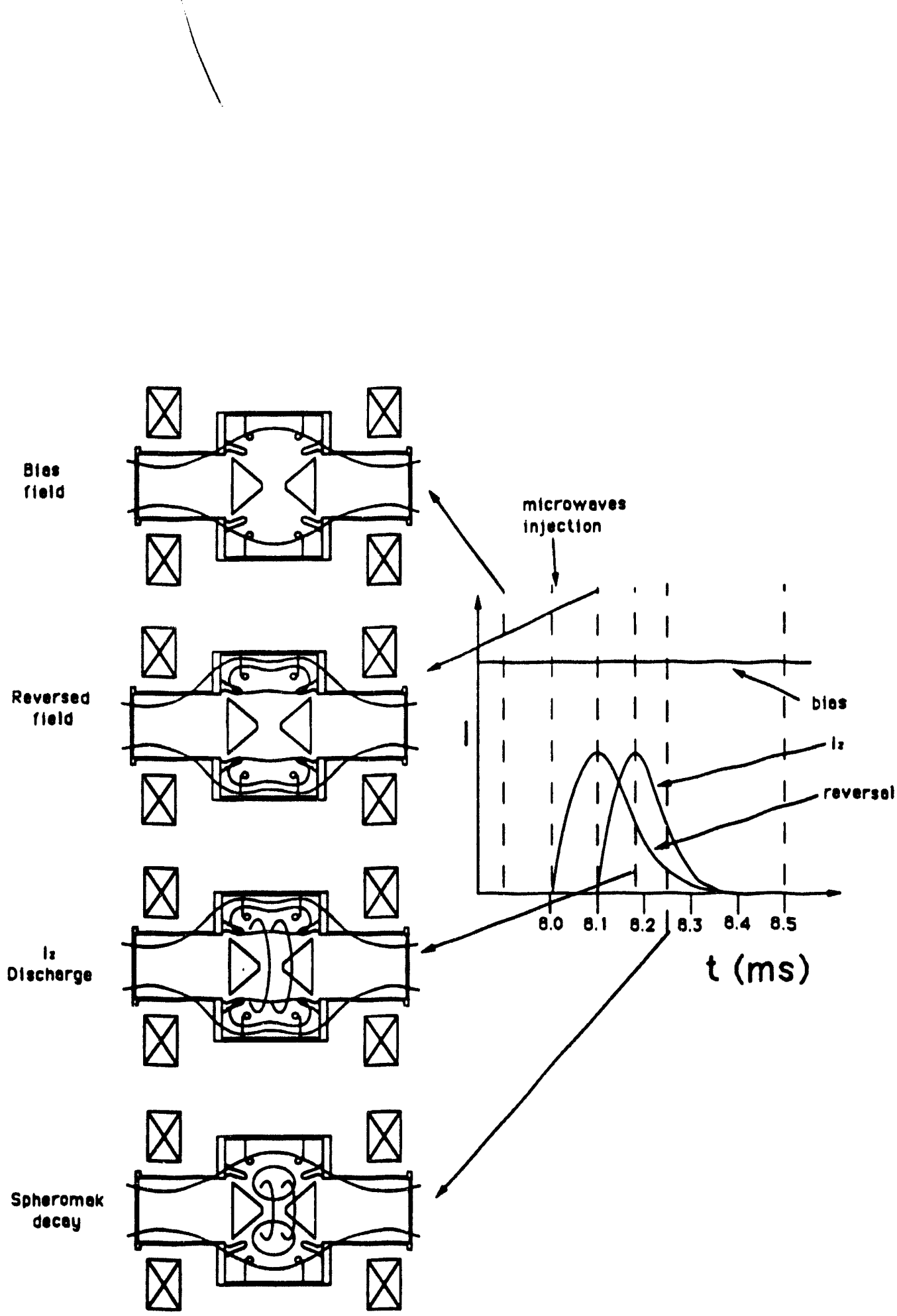

Figure 2.3: MS formation scheme. 
monitors using digitizers. Most raw data are immediately plotted for the operator to verify that the discharge was normal. After a sufficient number of cleaning shots a data scan can take place. Backups on magnetic tapes are performed on a regular basis. 


\section{Chapter 3}

\section{General Diagnostics}

\subsection{Vacuum Diagnostics}

The quality of the machine vacuum not only depends on the pumping speed of the different pumps but also depends on the cleanliness of the walls and internal parts. All surfaces that have been in contact with air will outgas for some time when brought under vacuum.

Each port is vacuum sealed with rubber O-rings or copper gaskets compressed between a flange and the port. Most ports using O-rings and most probes inserted in the machine are double-pumped. Even when the best cares are taken, real leaks do occur. Three diagnostics are availabie to monitor the vacuum and to pin-point the cause of leaks. 
Vacuum gauges monitor the base pressure obtained. The pressure is read everyday as part of the machine start-up process. A reading substantially higher than the previous day indicates a pump malfunction or a leak. A thermocouple gauge is used for pressures above $100 \mathrm{mT}$. In the 1 to $100 \mathrm{mT}$ range, a convectron gauge is useful to measure the chamber fill pressure and the after-shot pressure. Finally, for pressures below $10^{-4}$ Torr, a CVC ionization gauge is used.

A Residual Gas Analyzer (RGA) allowed the monitoring of different gases. It is a mass spectrometer scanning the partial pressures at different atomic mass numbers. The RGA is useful to see the effect of a particular pumping method on specific gases like water vapor, oxygen, hydrogen, etc... Heavy molecules $(a . m .>40)$ were interpreted as oil vapors, probably coming from the purnps.

A helium leak checker was used to identify sources of air leaks in the vessel.

\subsection{Magnetic Probes}

A changing magnetic flux through a coil will induce a voltage in the coil proportional to the flux rate of change. An array of coils in a glass tube and covered with a thin (.001") stainless steel jacket is inserted in the plasma and measures the flux rate of change along the $r, \phi$ and $z$ directions at several pustions simultaneously. After the probe has been calibrated with a Helmoltz 
coil, the signal becomes a measure of the rate of change of magnetic field. The signal is integrated using a low pass $\mathrm{RC}$ filter, reducing the frequencies that are higher than $1 / 2$ the sampling frequency of the digitizers. Corrections were made numerically for the non-linearity of the integrator. Also the value of the DC (i.e low frequency) bias field is added to the probe signal. A straight probe radially inserted could measure $B$ as a function of radius in the midplane and was used as a general monitor indicating the presence of instabilities in the magnetic field and the lifetime of the plasma. Figure 3.1 shows a typical trace of the toroidal and poloidal field in the midplane as measured by the probe.

A L-shaped probe was used to scan the plasma. Depending on its orientation, a $(r, \phi)$ scan in the midplane or a $(r, z)$ scan could be performed. Figures 3.2 and 3.3 show results from those two scans for different times. Complete scans for different conditions were taken [21]. Early data revealed a shift of the plasma. We found that the cause was the interaction of the plasma with the bare reversal coil, as explained in section 2.1. After insulating the reversal coil, the subsequent magnetic scan verified that the shift problem was solved. Other magnetic probes indicated the presence of poloidal currents near the walls, and its disappearance when the glass plates were installed.

\subsection{Rogowski probe}

Rogowski loops are used mainly outside the vessel to monitor currents. Two Rogowski loops are used to measure the total current feeding the positive and negative electrodes. Rogowski loops also monitor the current in each reversal 

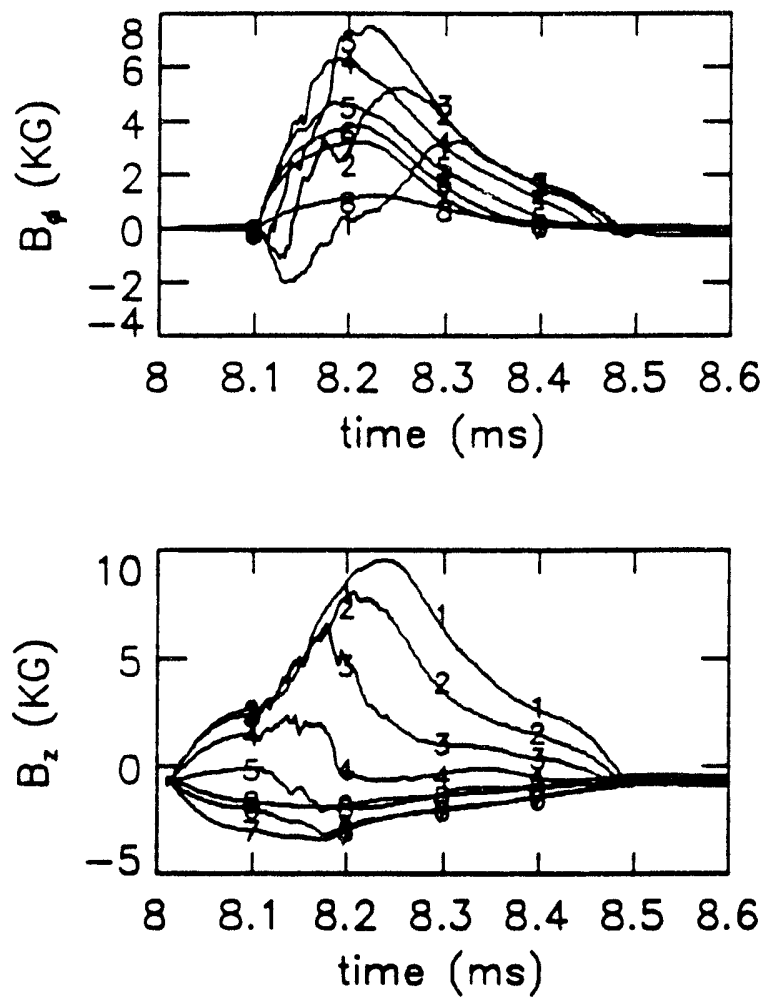

Figure 3.1: Typical toroidal and poloidal magnetic traces in the midplane for shot 5475 . Locations \# $1-6$ are at $r=(7.18,13.52,19.88,26.23,32.58,38.92$, $45.28,51.63) \mathrm{cm}$.

coil. Figure 3.4 show typical results for those currents. There is also a pair of Rogowski loops monitoring the current inside and outside the vessel for a particular electrode. The difference represents current flowing back from that particular electrode to the wall. 


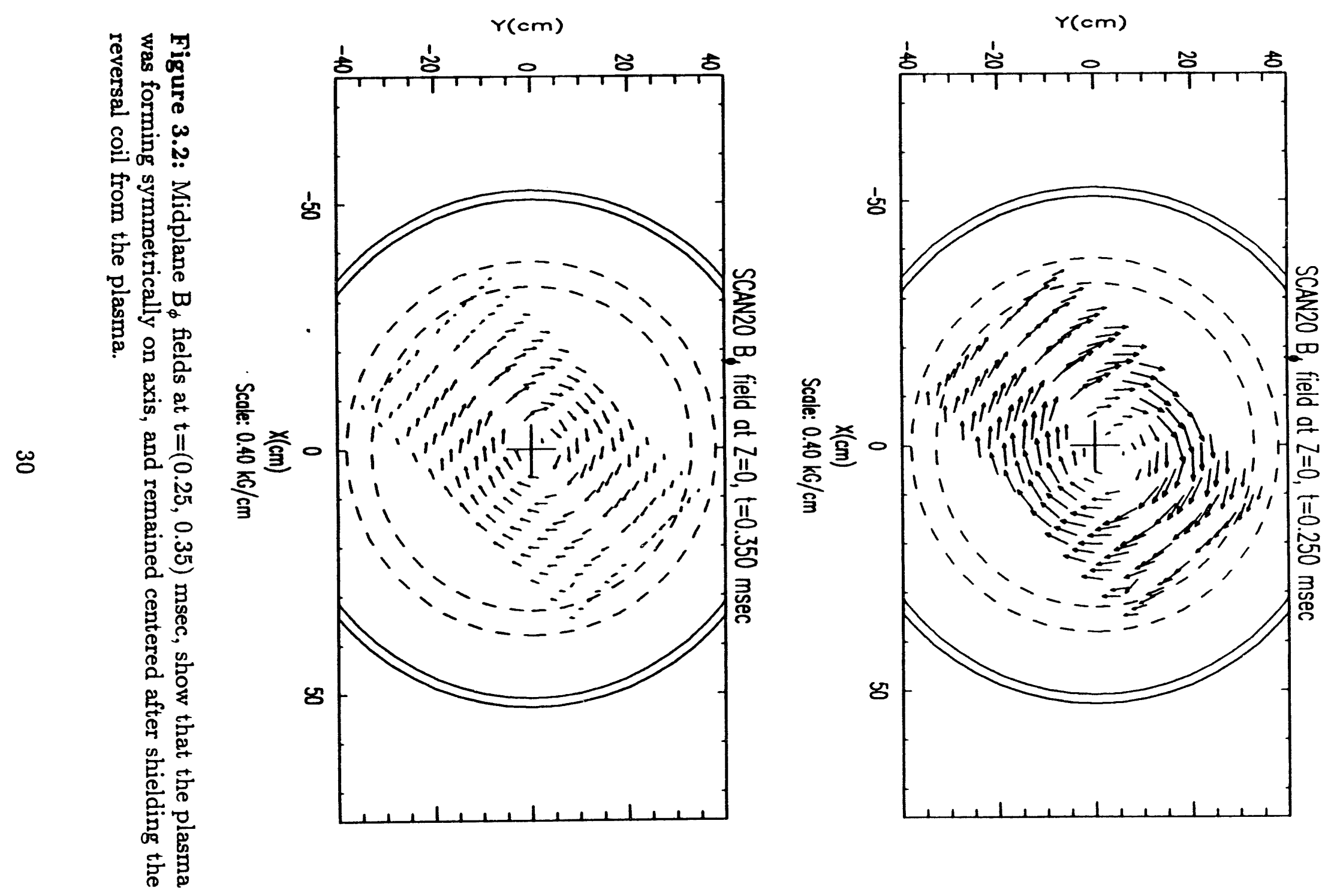



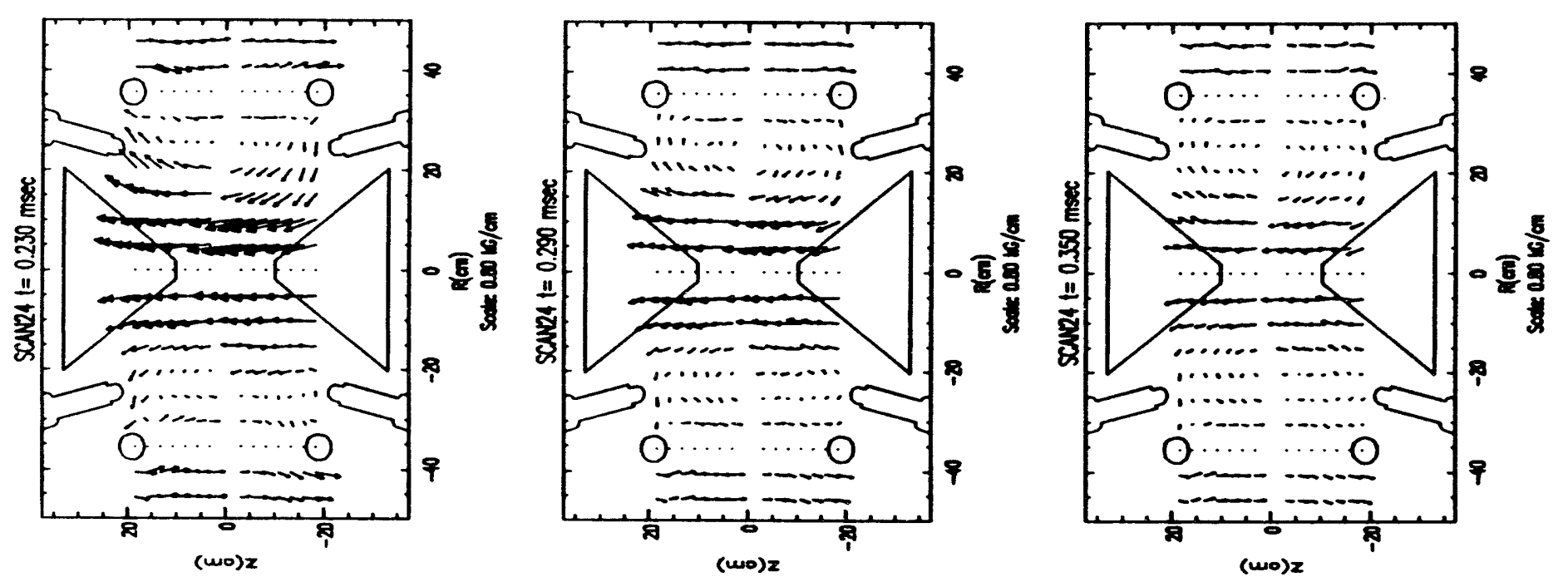

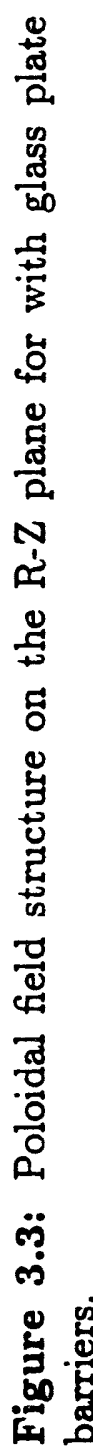
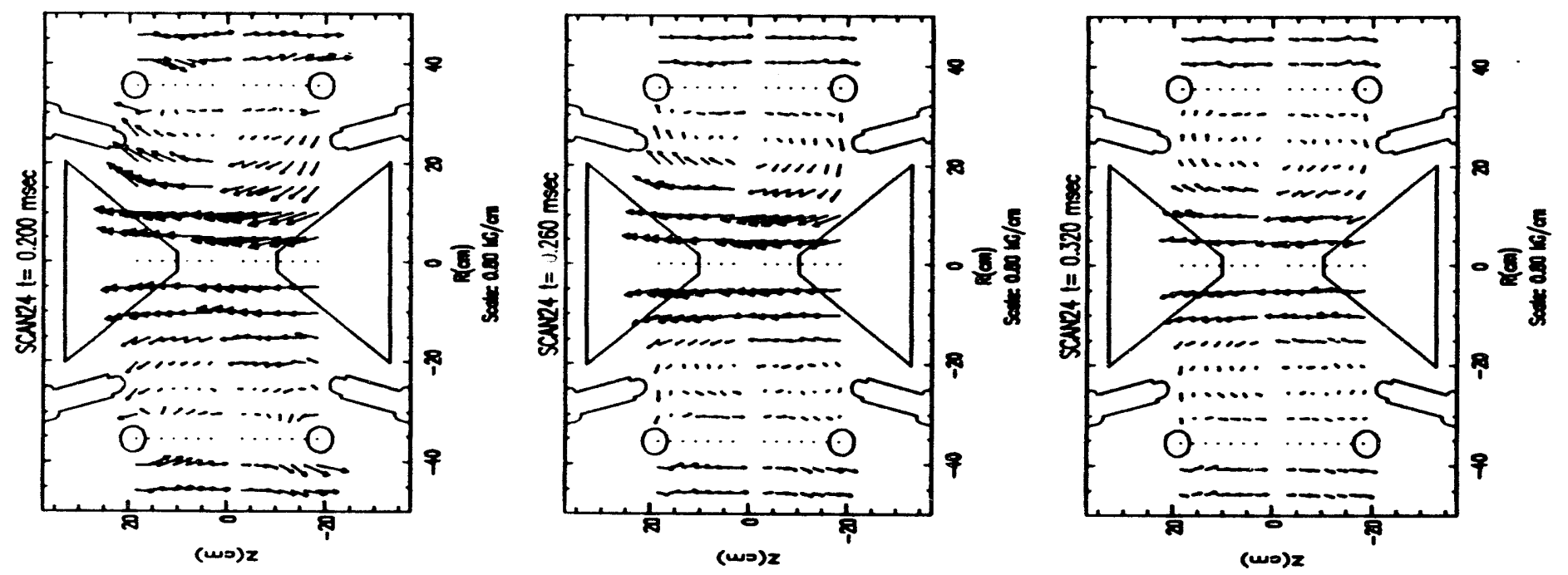

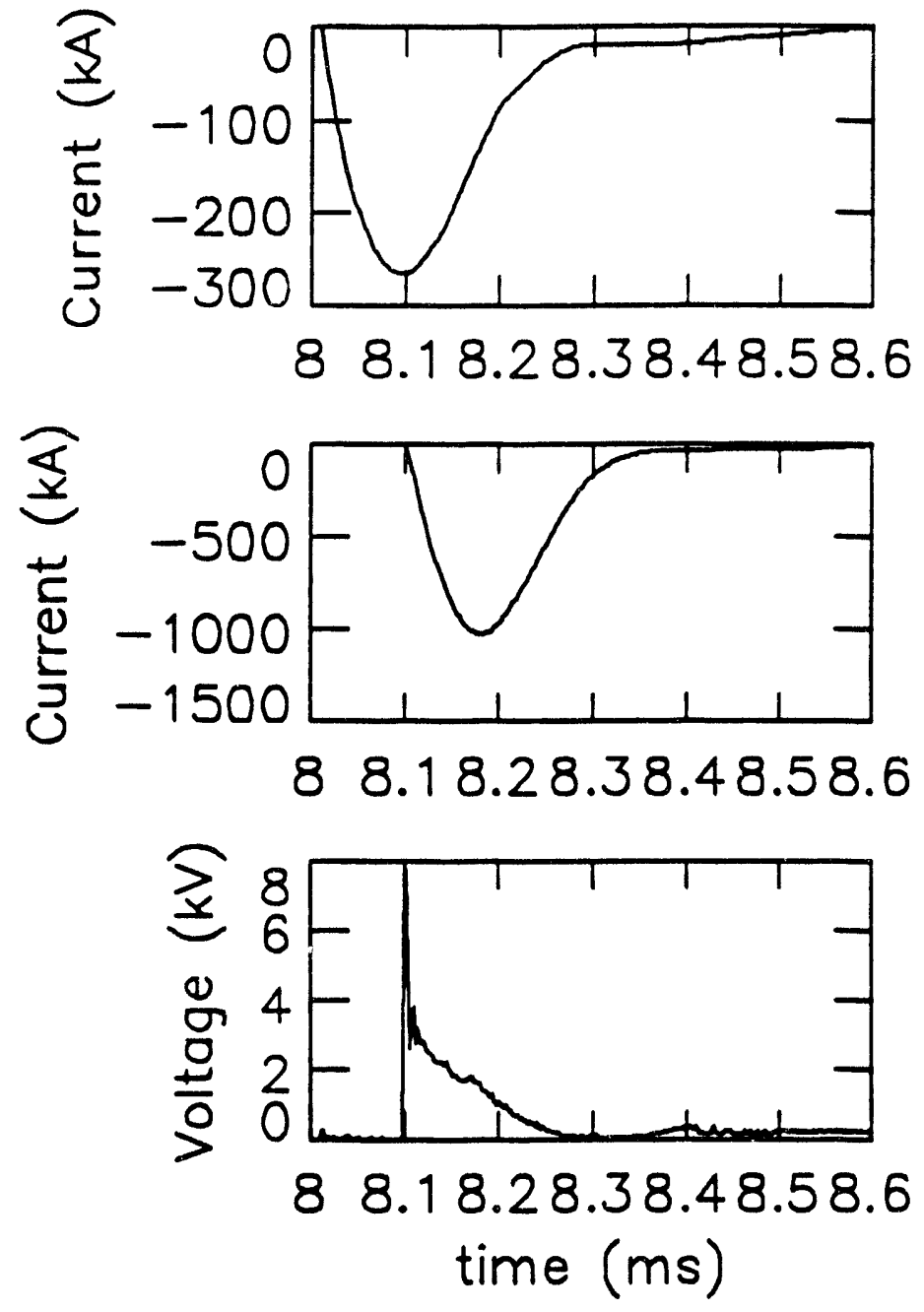

Figure 3.4: Top: Trace of the reversal current for shot 5475. Middle: Trace of the $I_{z}$ current for shot 5475. Bottom: Trace of the $I_{z}$ voltage for shot 5729 . 


\subsection{High voltage probe}

The voltage between each set of electrodes and ground is measured by a high voltage probe. The probe is a voltage divider, $\mathrm{RC}$ compensated and properly shielded. Figure 3.4 shows a schematic of the probe and its typical signal is shown in Fig. 3.4.

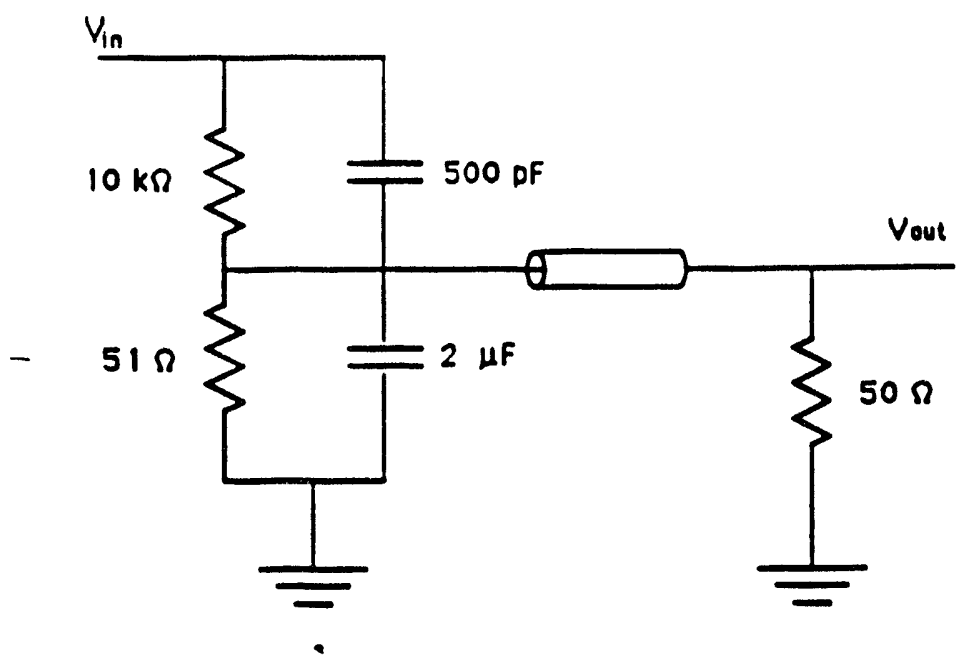

Figure 3.5: High voltage probe circuit diagram.

\subsection{Interferometry}

Because the plasma has a different index of refraction than air, a laser beam passing through it will have a different phase shift than a laser beam travel- 
ling the same distance in air. We can take advantage of that by superposing the probing and the reference beam on a target. If the beams are spatially coherent, the difference of phase shift will produce interference [29]. By monitoring the resulting intensity of interference, one can calculate the line averaged plasma electron density,

$$
\frac{\Delta \phi}{2 \pi}=2.84 \times 10^{-18} \bar{n}_{e} L
$$

using cgs units. The phase shift is related to the observed intensity through a function of the form

$$
I \alpha \cos (\Delta \phi)+1
$$

Every time $I$ goes through a minimum or a maximum, there can be an uncertainty in knowing if $\Delta \phi$ is increasing or decreasing. Equation 3.2 is multi-valued in density. The ambiguity is resolved through quadrature interferometry [30]. The scene beam is polarized at 45 degrees and the reference beam is circularly polarized. Once they are merged, the new beam has its horizontal component 90 degrees out of phase with respect to its vertical component. When one component has its maximum intensity, the other has its minimum. Two detectors are assigned to read the intensities of these two components. When an ambiguity occurs for one detector, the behavior of the second signal can resolve it. The interferometer set-up is mounted on an air supported optical tatle. 4 beams are simultaneously probing the plasma. A total of 8 lines of sight could be used. Plasma density mea urements showed that the electron density is higher than expected from the initial puffed gas 
pressure [22]. Figure 3.6 shows typical line averaged density profiles obtained. The data were inverted using a Bessel function method to obtain the electron
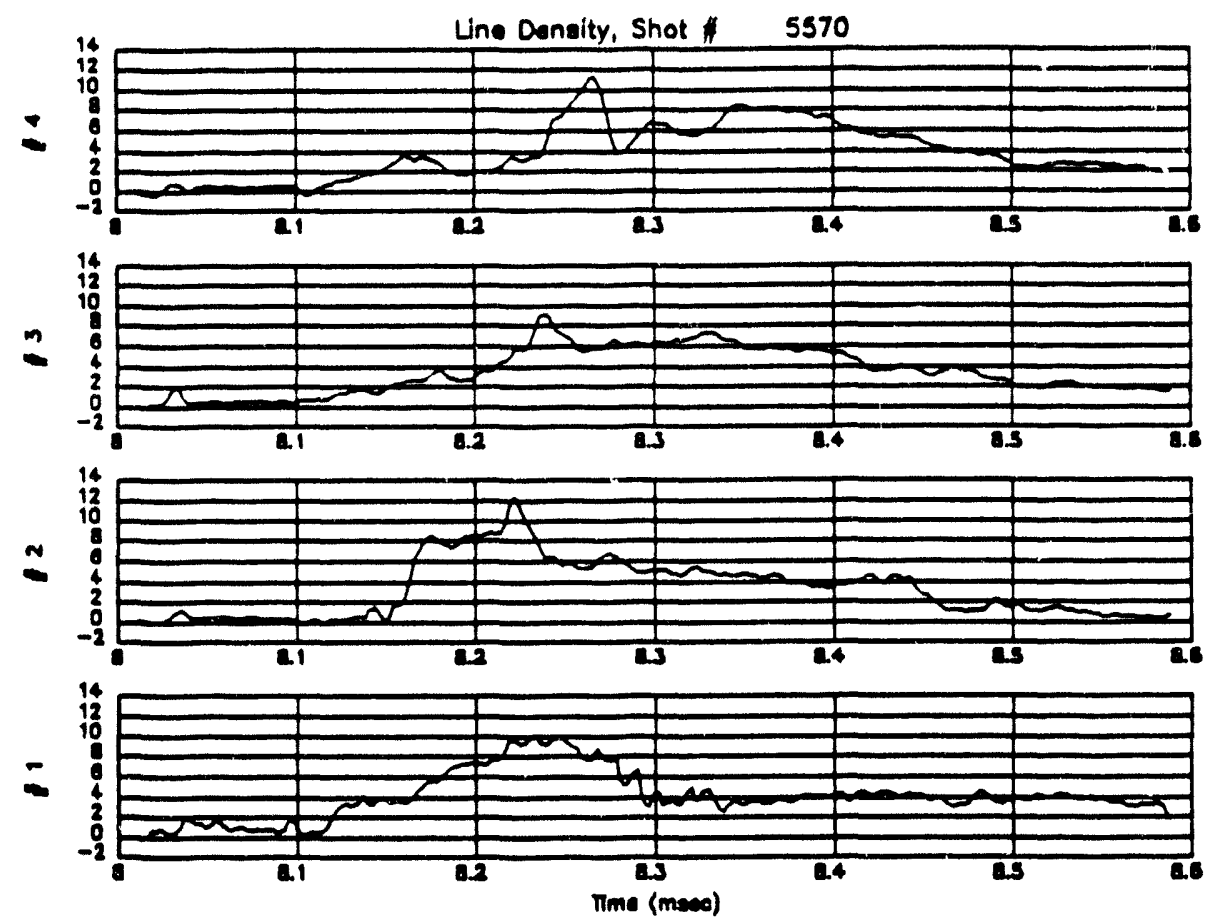

Figure 3.6: Line average electron densities at different chord parameters with glass plate barriers. The chord parameters \# 1-4 are at $(0,7.62,19.05,26.62) \mathrm{cm}$.

density as a function of radius.

\subsection{Langmuir Probe}

By measuring the I-V profile of two metallic probe electrodes in contact with the plasma, one can extract the electron temperature and ion density [?]. 
As the vessel ports allowed interferometric measurements up to $r=30$ $\mathrm{cm}$, Langmuir probe measurements were complementary in order to obtain the electron density near the walls. Because of the indetermination in $Z_{\text {eff }}$, and in the absolute calibration of the probe, the obtained electron density was multiplied by a factor to match the measured density with the interferometer.

\subsection{Bolometry}

Bolometers and pyroelectric detectors are used to detect the total power emitted by radiation. It is found that most of the power radiated is in the range of 500 to $1600 \AA$.

A bolometer is sensitive to electromagnetic radiation, neutrals, and charged particles from a plasma. Its front metallic surface absorbs the incoming energy. The change of temperature affects the resistance of a resistor mounted on the back of the surface. A measure of the change of resistance is then proportional to the total energy absorbed by the detector. When sufficiently collimated, we can assume that the contribution of charged particles is negligible. Because of the small thermal conductivity of the insulating layer between the front plate and the resistor, the response time of the detector is rather long: about $100 \mu \mathrm{s}$. This is not sufficient to get detailed information of MS time history, but it is useful to get the total energy radiated. Figure 3.7 shows the inverted energy density as a function of radius. A total of $80 \pm 30 \mathrm{~kJ}$ radiation is obtained from those measurements [?]. The total energy delivered by the $I_{z}$ is about 80 $\mathrm{kJ}$ and the reversal coil is driven by a $200 \mathrm{~kJ}$ energy bank. This implies that 
only a small fraction of the reversal magnetic energy stays in the plasma.

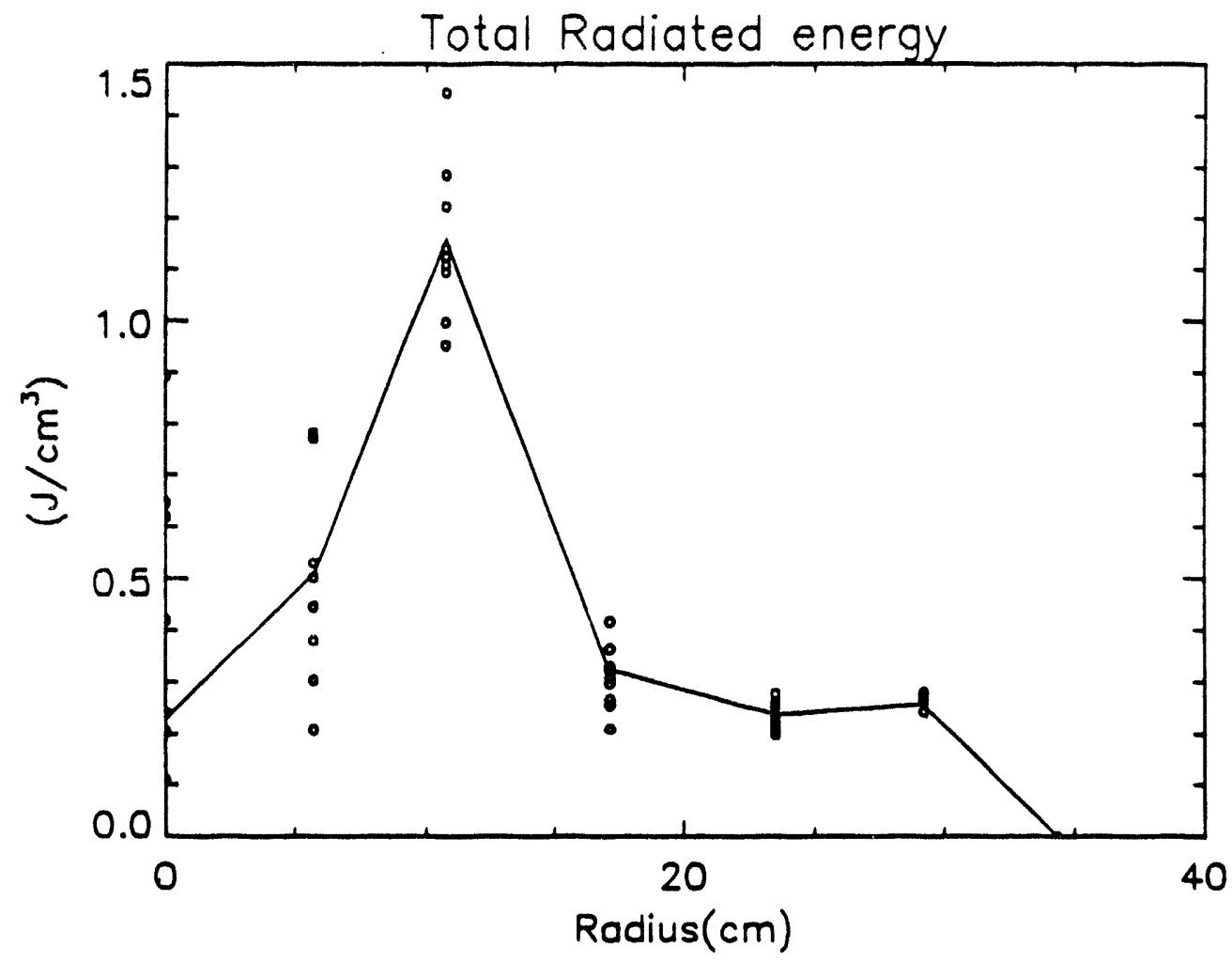

Figure 3.7: Radiation power density integrated over time.

Pyroelectric detectors were also used to measure the radiation power emitted by the plasma. Each detector consists of a thin ferroelectric crystal, $\mathrm{LiTaO}_{3}$, which, below the Curie temperature of 610 degrees $\mathrm{C}$, has a temperature dependent spontaneous polarization. A change of temperature will create a displacement current in the crystal and a compensating current flows in the external measuring circuit. The side facing the plasma has a grounded gold electrode that will absorb all radiation from the visible to UV range. In this device, the time response is a few microseconds, making it an appropriate detector for the MS experiment. Measurements were made with and without 
a lithium fluoride window. The window cuts all radiations below $1100 \AA$. If we rule out neutral and charged particles hitting the detector, then it appears that most of the energy comes from below $1100 \AA$. The pyroelectric signal versus chord parameter for different times is shown in Fig. 3.8 [?]. A small asymmetry exists between the upper and lower parts of the scan.
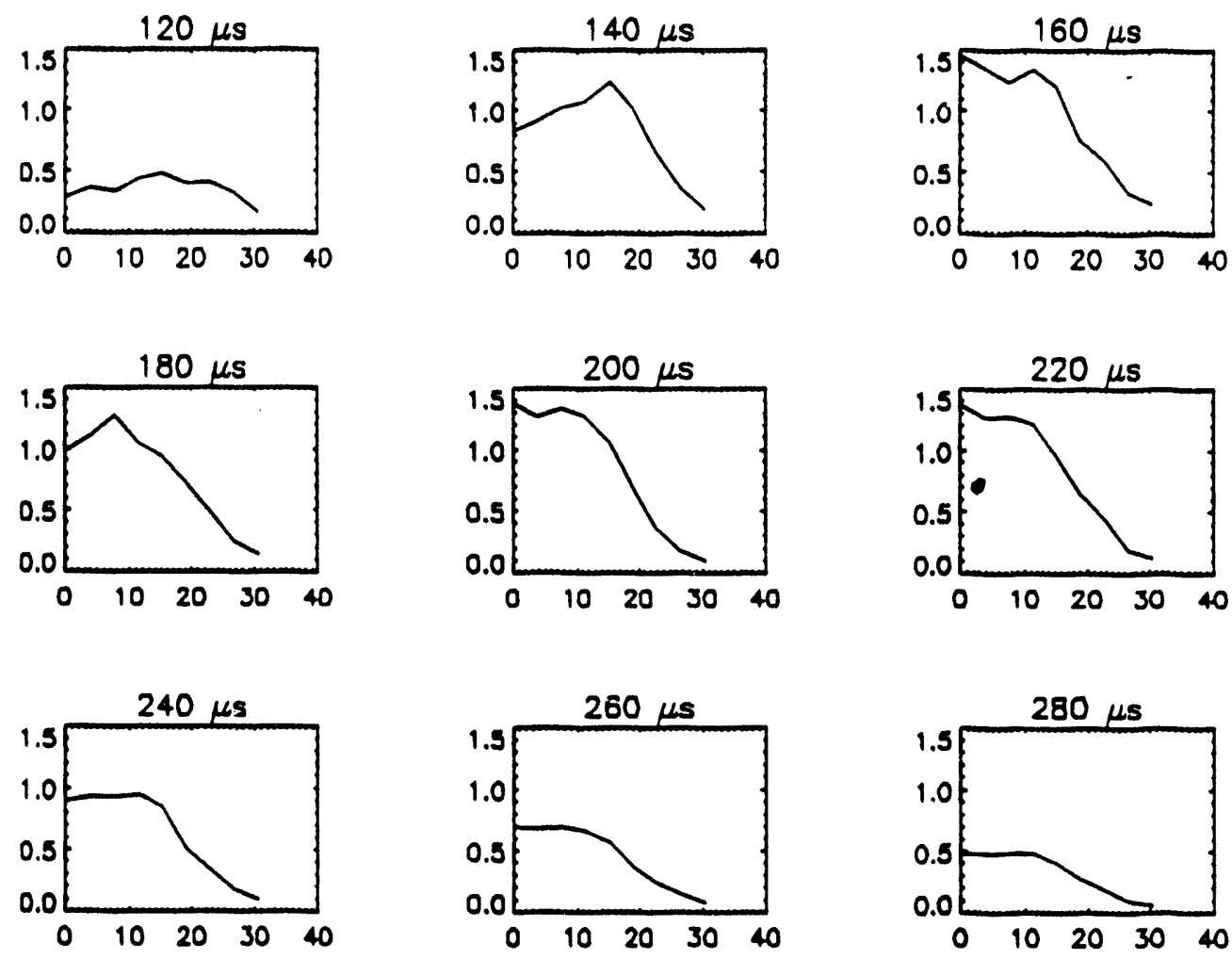

Figure 3.8: Pyroelectric signal as a function of chord parameter for different times. 


\section{Chapter 4}

\section{Spectroscopic Diagnostics}

\subsection{Monochromators}

Two Jarrell Ash quarter meter monochromators were used with photo-multiplier tubes (PMT) to monitor spectral lines in the plasma. The device is sensitive from ultraviolet to near infrared regions. The PMT signal is delayed by about $100 \mathrm{~ns}$ and has a rise time in the order of $10 \mathrm{~ns}$ both of which are better than we need. Figure 4.1 shows the PMT set up to monitor impurity lines.

The CIII (2298 $\AA)$ line was monitored continuously as it gave some indication of plasma cleanliness. There was a correlation between the electron density measured by interferometry and this signal. The first few shots of the day always showed higher levels of CIII than subsequent shots. The second 


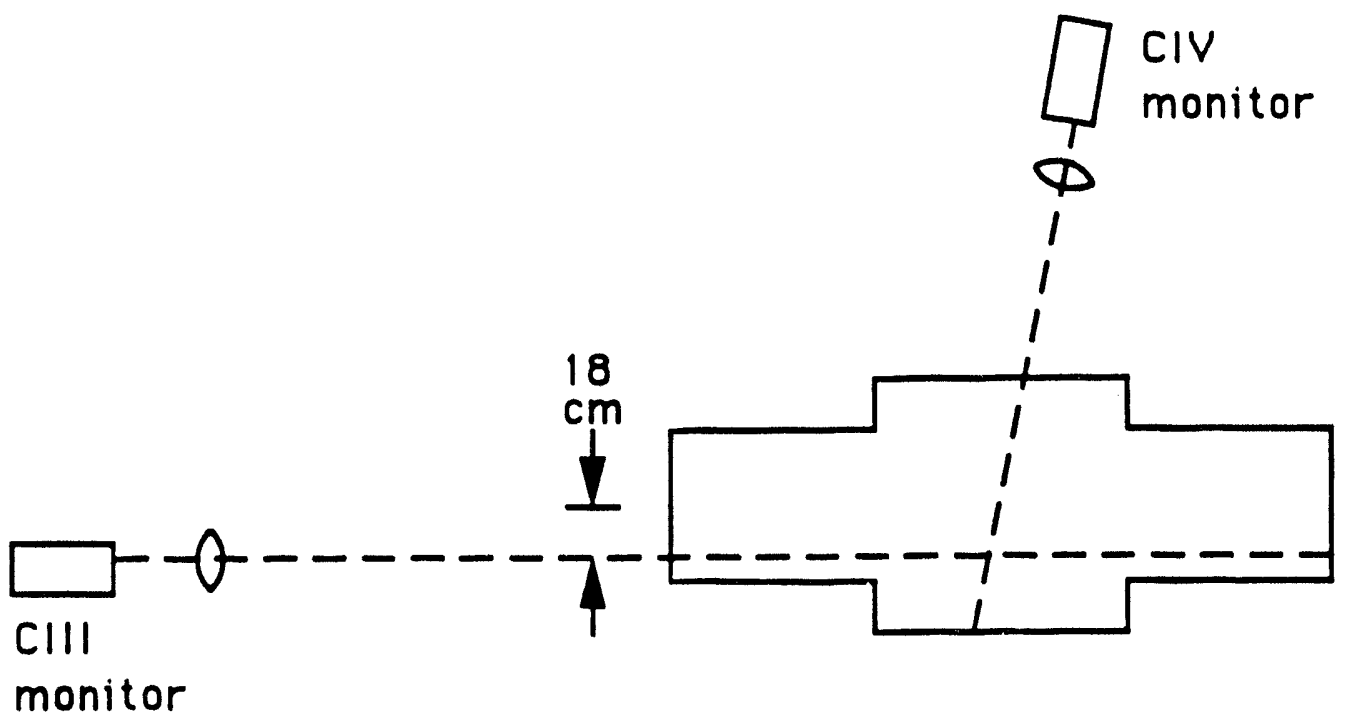

Figure 4.1: Line of sights used to monitor CIII and CIV impurity lines with the monochromators

monochromator was used to monitor CIV (5508 $\AA$ ). For our plasma temperature, this line intensity was more dependent on electron temperature than CIII was. We always waited to see reproducibility of the CIII and CIV line integrated emissions before starting a scan. Figure 4.2 shows the signals obtained for clean and dirty plasma conditions.

A rotating mirror was installed inside the vessel to scan the plasma at many angles as shown in Fig. 4.3. Using a monochromator we obtained a time history of the $\lambda=2297 \AA$ line of CIII and the lambda $=5812 \AA$ line of CIV for different chord parameters. The data could be inverted to obtain the radial distribution of those species as a function of time and radius. An Abel 

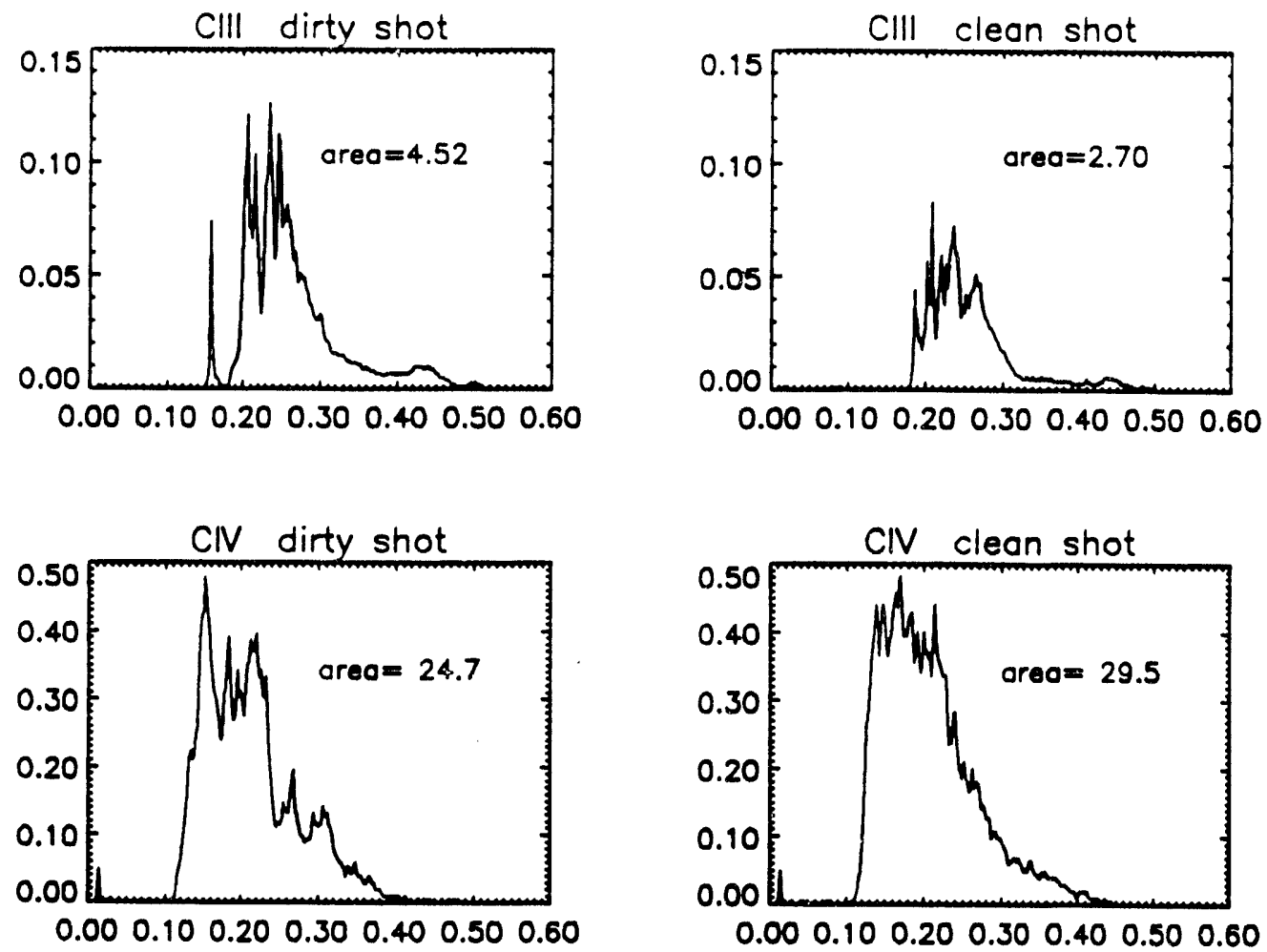

Figure 4.2: CIII and CIV signals under clean and dirty conditions.

inversion method was used. A calibration of the optical system was obtained with a tungsten lamp, giving the relative sensitivity of the instrument for those two wavelengths. From the ratio of line intensities we could obtain the electron temperature ${ }^{1}$. As the electron temperature rises, the atoms become more and more ionized. Typically for a given electron temperature, two ionized stages will coexist simultaneously. As the temperature is raised, the lowest stage begins to disappear while a higher stage begins to appear. From atomic theory, if we know the oscillator strength of the transition, the population of the upper states and the population of the ionization stages, then for a given electron

\footnotetext{
${ }^{1}$ The theoretical relationship between electron temperature and line ratio was graciously calculated by Dr H.J. Kunze.
} 


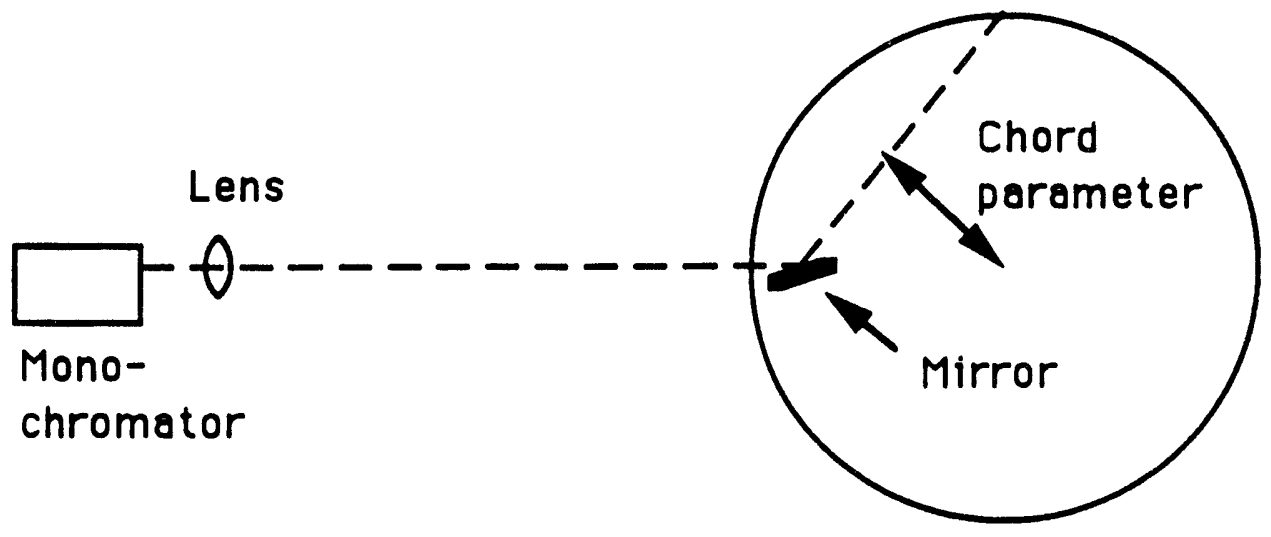

Figure 4.3: Optical set-up to scan the chamber.

temperature, one can calculate the relative emission intensities of the two lines [33]. Figure 4.4 shows the electron temperature obtained as a function of space for different times from the inverted emissions and assuming that transients are not important.

\subsection{VUV spectrometer}

A one meter normal incidence vacuum ultraviolet (VUV) spectrometer was used to monitor the spectrum between (500 and $1500 \AA$ ). Since bolometry indicates that most of the radiation comes from that region, the purpose here 

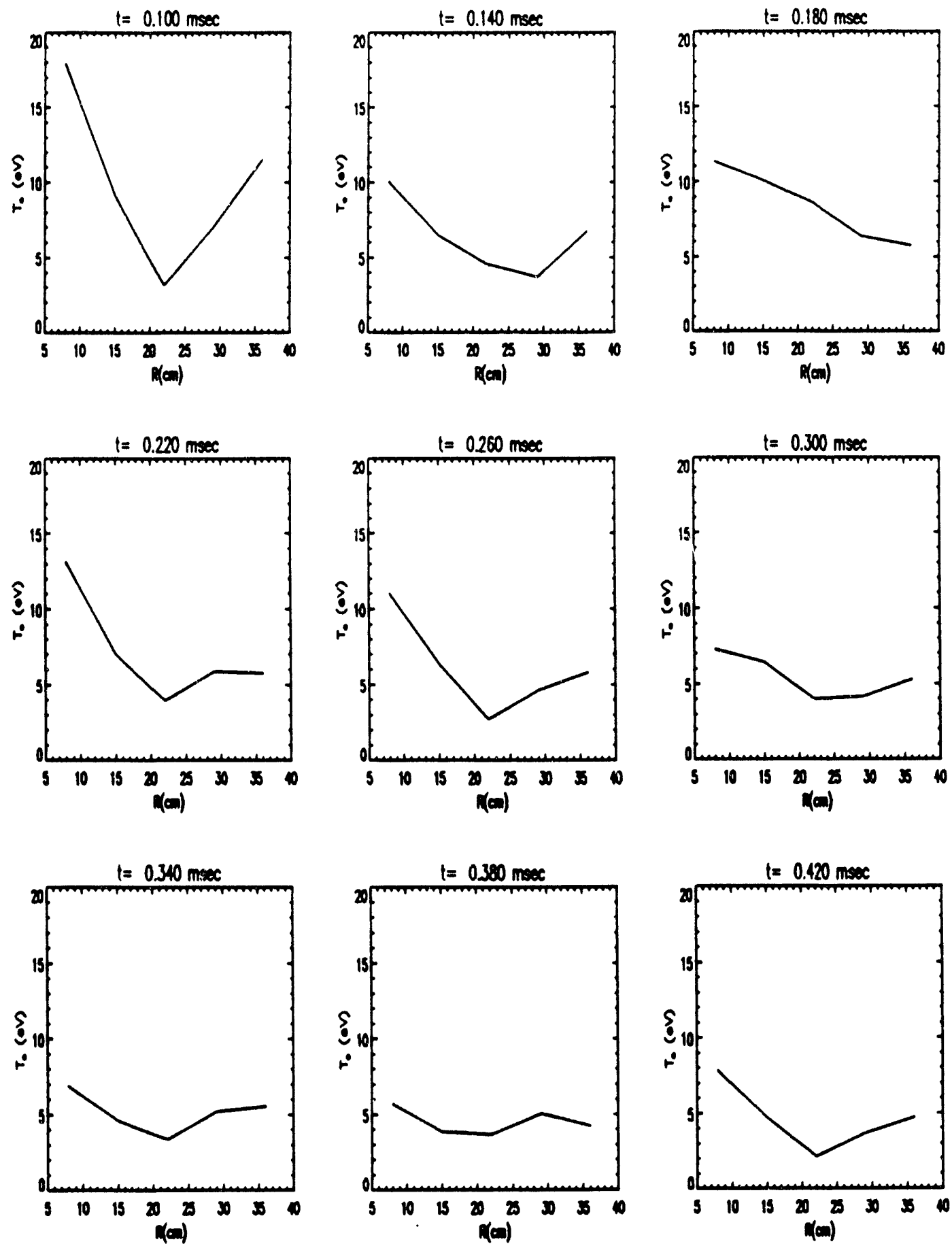

Figure 4.4: Radial behavior of the electron temperature obtained from CIV to CIII line ratio. 
is to identify the contribution of each element to power loss by radiation. The spectra were time integrated on a photographic film. Looking in the midplane. lines of carbon, oxygen, nitrogen and fluorine were mainly observed. The highest ionization stage observed was OVI, indicating a lower bound for the electron temperatures of $15-20 \mathrm{eV}[37]$.

\subsection{Linear OMA}

A linear Optical Multichannel Analyzer (OMA) was used to monitor impurities emitting in the visible and near UV. Incoming photons strike a photocathode and the emitted electrons are intensified by a Microchannel Plate (MCP), accelerated to a phosphor screen and produce secondary photons. These photons are detected by a Linear Silicon Photodiode Array, called a reticon OMA. The model used has 700 active photodiodes corering about 20 $\mathrm{mm}$. The detector is gated for a few micro-seconds, meaning that light is accumulated during only a predetermined window in time. The line of sight is parallel to the machine axis, $18 \mathrm{~cm}$ off axis. A quarter meter monochromator with a low resolution curved grating was used to disperse the light. The entire visible impurity spectrum could be obtained in one shot, from 2000 to 6000 $\AA$. One could identify the main impurities and the effects of machine modifications. A second OMA mounted on a higher resolution spectrometer could provide more detailed information on the impurity line shapes.

Figure 4.5 shows typical results just after peak of $I_{z}$. It appears that even if the plasma is surruunded by stainless steel, iron is not a major source of 
impurity radiation for our temperature and regime of operation. One major impurity is oxygen and could be coming from $\mathrm{O}_{2}$ molecules and water vapor absorbed in the walls. Another major impurity is carbon. Its source could be $\mathrm{CO}_{2}$ from the walls and/or pump oil. We also observe the presence of aluminum ions. They are suspected to originate from alumina ceramic (which is aluminum oxide) used to cover the electrodes as they show signs of surface currents.

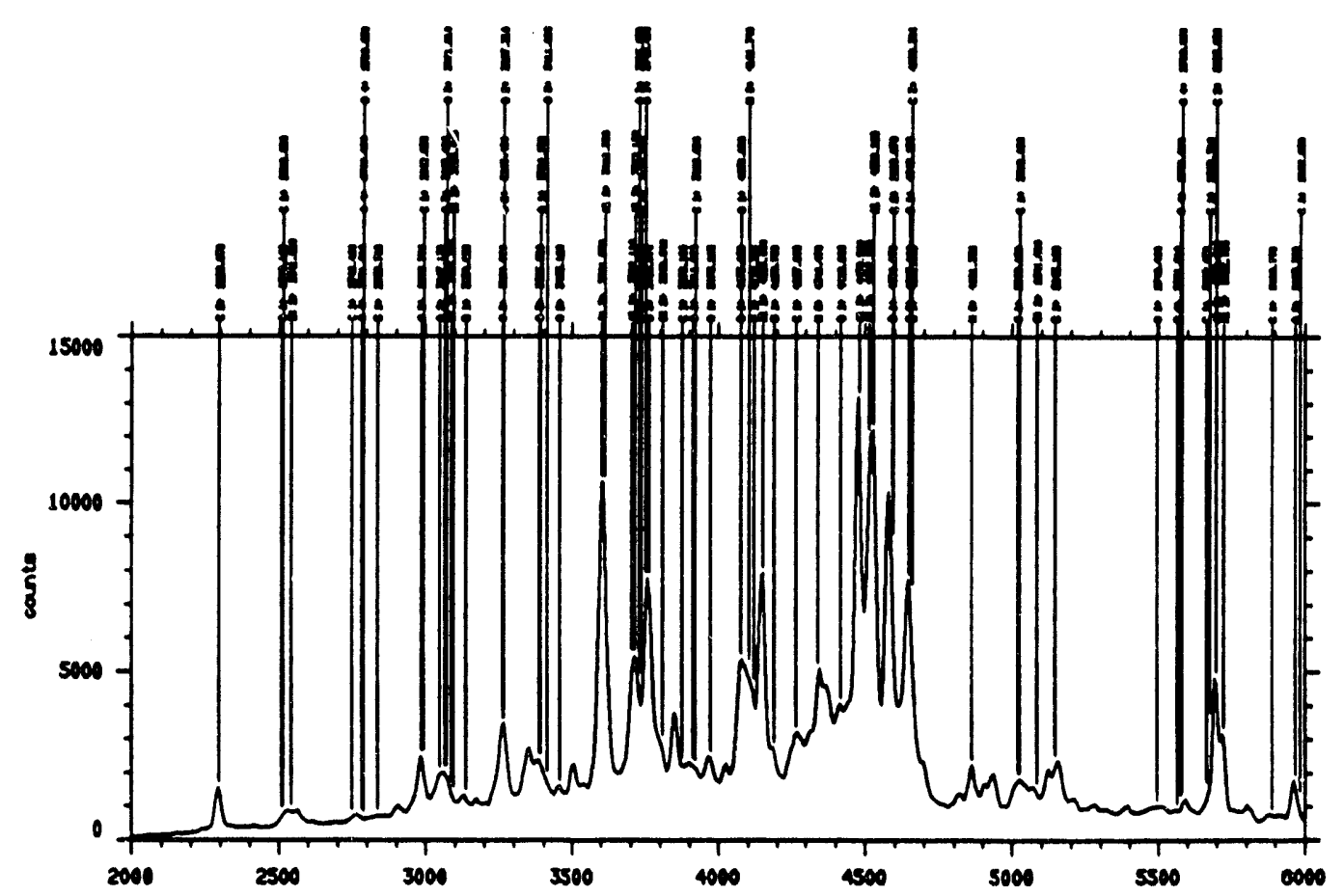

Figure 4.5: Impurity profiles obtained with the linear OMA. 


\subsection{2-D OMA}

The main results of this thesis will be shown in the next chapter and were taken with a two dimensional OMA. It is a $500 \times 500$ array vidicon detector of the ISIT type. As light strikes the front surface, a UV scintillator converts any UV light into the visible range. Those photons are converted into photoelectrons when they strike the photo-cathode. The electrons are reconverted into light after passing through an electrostatically focused image intensifier. This intensification process is repeated in a second stage before being detected by a two-dimensional array of photodiodes. The silicon photodiodes are read and recharged by an electron beam continuously scanning the wafer. See Fig. 4.6. The current necessary to recharge it is collected by a preamplifier. The signal is digitized by the controller and relayed to the console where it can be displayed and stored. In this experiment, the spectra were sent from the OMA console to the data acquisition computer into the database.

The sensitivity of the detector is about 10 photons per count in the visible and it drops to about 100 photons per count in the near UV (2000 to 3000 $\AA)$. The OMA is gated by applying a high voltage square pulse to the first intensification stage. This allows to gather data only for the desired period of time, typically $20 \mu s$. On the other hand it was also used in continuous mode during calibration and alignment procedures. By itself, the OMA is just a digital camera. A small monitor connected to the controller provides a visual image of the illumination of the detector head. When mounted behind a spectrometer, the OMA provides spectroscopic information along its horizontal axis. See Fig. 4.7. Spatial information is obtained along its vertical axis as it 


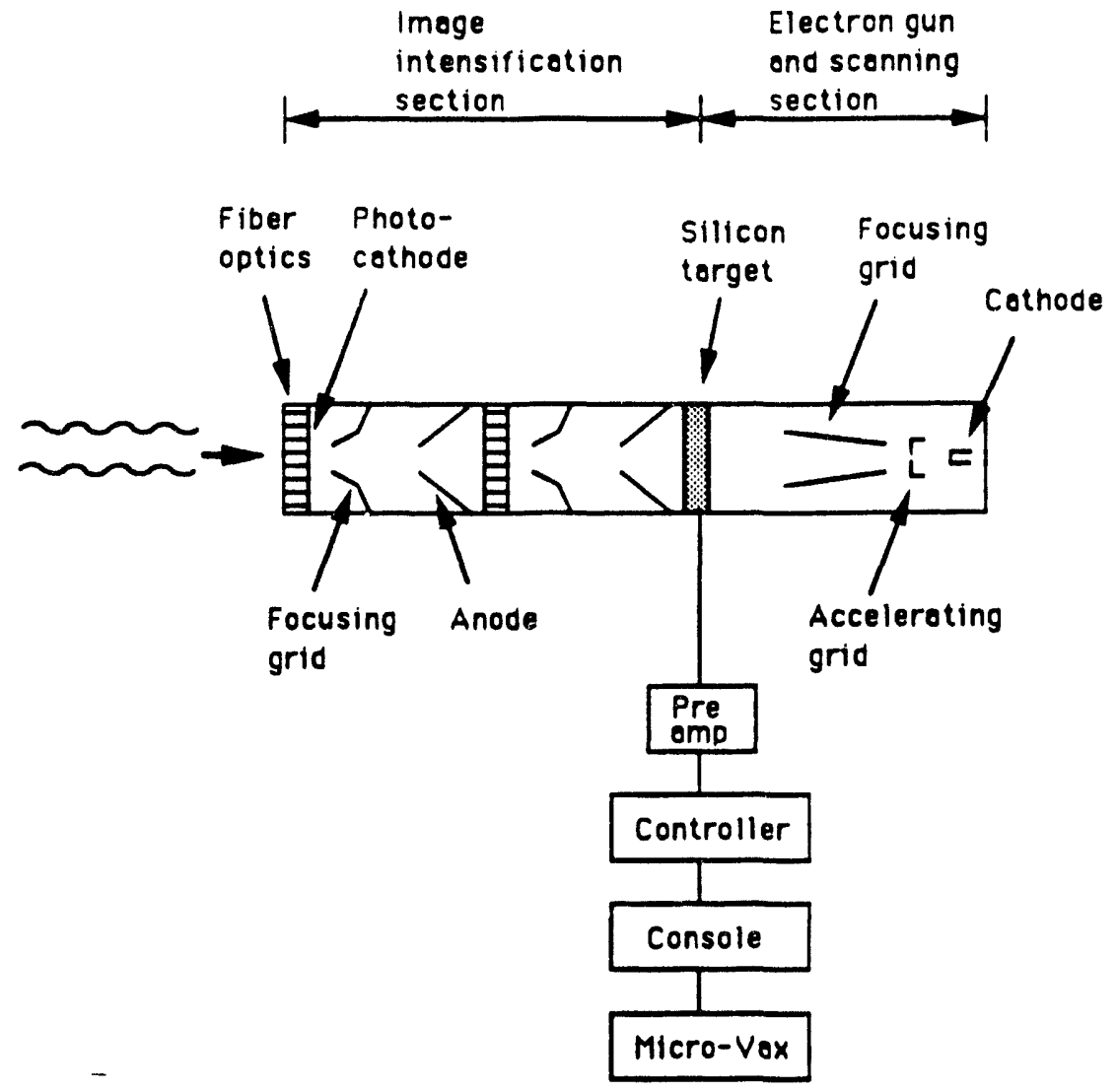

Figure 4.6: OMA head

is mapping the entrance slit of the spectrometer. A thorium lamp was used to calibrate the OMA in wavelength. In the visible and near UV range, the entire spectrum of thorium lines was identified and could be used for future wavelength calibration when identifying impurities.

Because only 16 kbytes of memory were available on the OMA console, not all $500 \times 500$ pixel intensity counts can be collected. The pixel data have to be grouped beforehand. When used to analyze spectral line profiles, the full 500 channels are kept horizontally and data are grouped together vertically, 

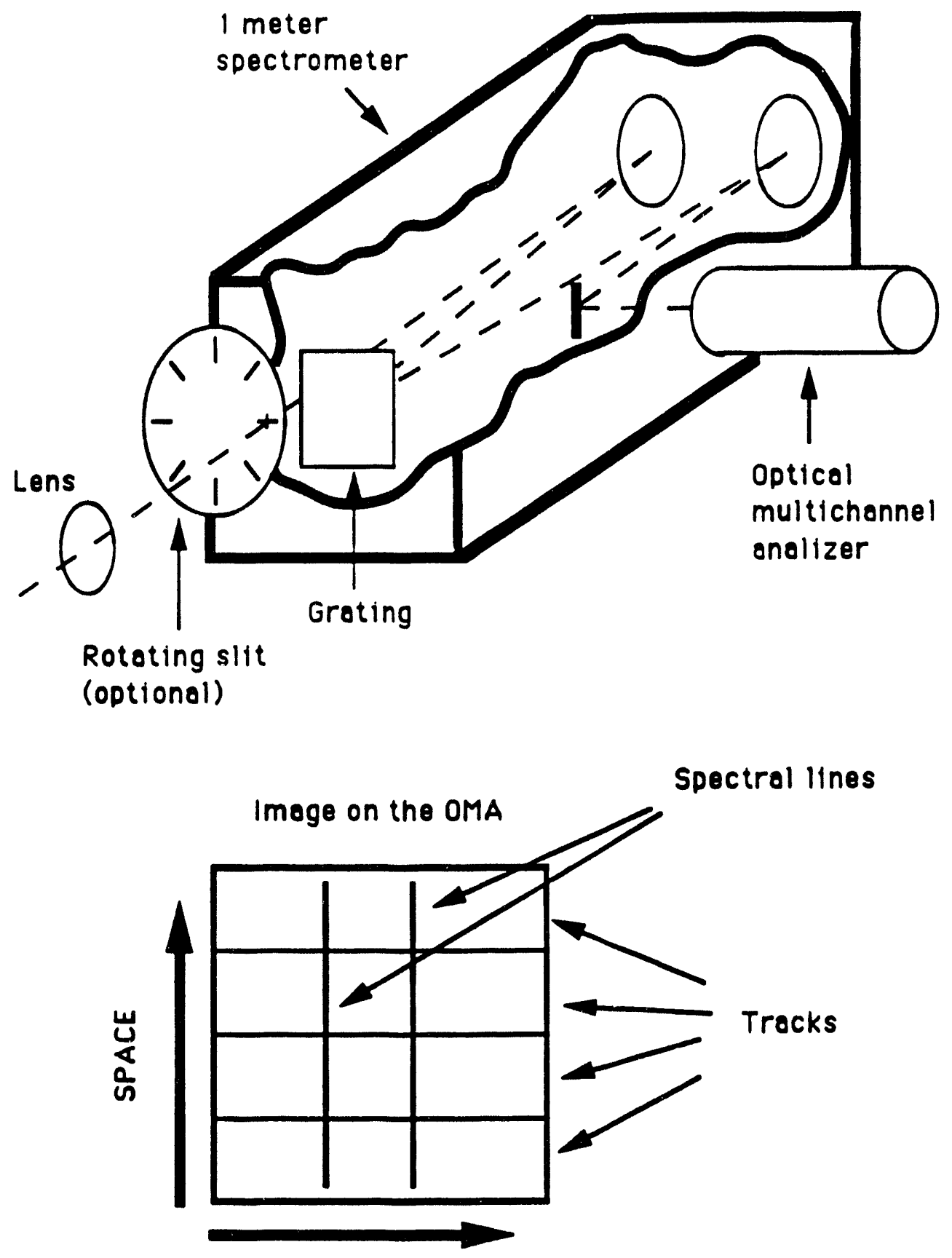

WAVELENGTH

Figure 4.7: Diagram of the OMA mounted on a $1 \mathrm{~m}$ spectrometer. 
forming several horizontal tracks. Tp to 16 tracks could be used. When taking digital 2 dimensional pictures, the data are grouped in 100 channels $\times$ 80 tracks.

\subsubsection{Gating the OMA}

The voltage applied should be a square pulse with rise times much shorter than the length of the pulse, to minimize the distortion of the collected signals. For this purpose, krytron switches are used. They have rise times much shorter than a $\mu s$ and can handle cur high voltages. A capacitor is charged to about 1450 volts. At firing time, a TTL trigger is converted into a 300 Volt pulse and triggers the first krytron. At that point about 1450 volts is applied to the OMA head. Later, a second pulse triggers the second krytron which grounds the output and terminates the pulse to the OMA head. Those circuits are shown in the appendix.

\subsubsection{Cooling the OMA}

The OMA has two sources of dark current noise, which is the thermal noise present when no light is hitting the head. The first one comes from the head itself. Thermal electrons from the intensifier stages can leak out of the semiconductor surface and will cause dark current to be read from the head. This dark current is non uniform across the head and can be reduced by cooling the head. The second source of dark current comes from the pre-amplifier in 
the OMA controller which is a separate unit. Since the same pre-amplifier is used for all channels this current appears as a constant background signal over the whole surface of the head and can be subtracted. A copper jacket was added around the OMA head. Flowing cold water through this jacket did not decrease the dark current noise substantially. However, when liquid nitrogen cold vapors were used instead, the noise coming from the OMA head becomes insignificant and a flat signal coming from the controller was the only dark current observed. A quartz window was added on the front surface of the OMA head and room temperature nitrogen gas was made to flow at a slow rate inside and around the head to prevent condensation. One drawback is that the OMA calibration kept changing with time. This is likely due to expansion and contraction of different parts of the head as the temperature keeps fluctuating. For that reason as well as other problems, cooling the OMA head was abandoned.

\subsubsection{Rotating Slit}

Although the detector head is gated for a short time $(20 \mu s)$, it takes about 10 milliseconds to read the integrated signal on the head and several seconds to store it on a disk. Therefore only one time window of information can be taken per plasma shot. When gathering preliminary data, it was found to be more important to obtain the time behavior of a specific line during the plasma discharge than its spatial dependence. To achieve this purpose, a rotating slit was added in front of the entrance slit of the spectrometer as shown in

Fig. 4.7. With a horizontal slit scanning the head vertically, different portions 
of the head are illuminated at different times. It is then possible to obtain time dependent signals. A disk with 60 slits was made to rotate from 2000 to $8000 \mathrm{rpm}$. For a 10 tracks configuration, this gave a time resolution varying from 50 to 12 microseconds. All the tracks are assumed to be illuminated by a homogeneous plasma. This condition is verified by removing the rotating slit and checking for uniformity of the data from track to track. A light sensitive diode was mounted behind the rotating slit with a laser illuminating it through the slits of the wheel. By monitoring the signal from the diode we could get absolute time resolution, since we can find which track is illuminated by the plasma when the diode receives laser light. The rotation frequency of the slits is also obtained from the diode signal. The OMA was gated for a slightly shorter time than the period necessary to scan the head such that each track is only exposed once. When taking a radial scan at different chord parameters, a set up similar to the one shown in Fig. 4.3 was used with the OMA.

The rotating slit was useful to gather preliminary data, because in a few shots many points in time could be obtained for a given chord parameter. However, the obtained times are random since the experiment is triggered independently of the angular position of the rotating slit. Also the angular velocity of the wheel tends to drift in time. Another disadvantage is that each track is looking at a slightly different portion of the plasma. For a more precise scan, we took data at a well defined time, for several simultaneous chord parameters, using a wide angle lens. 


\subsubsection{Wide Angle Lens System}

Because of the strong magnetic field generated by the bias coils, the OMA could not be brought too close to the machine without being affected. It is necessary to have an ensemble of lenses to fill the spectrometer grating with light and obtain the desired image magnification simultaneously. A simple ray tracing cornputer code was written to help in choosing the optimum focal length, lens diameters and lens locations. The result is shown in Fig. 4.8. This 3 lens system maps the entrance slit of the spectrometer to a line in the plasma. The image of the entrance slit is mapped by the lens L1 on L2. L2 is a field lens mapping L1 to L3. Finally L3 maps L2 to a line in the plasma. The actual system used is also shown in Fig. 4.8 and is somewhat different. The consequence of removing L3 is that some spatial resolution is lost. L2 is mapping $\mathrm{L} 1$ to an image of $1 \mathrm{~mm}$ diameter due to the geometry. When removing L3, only the light entering this small diameter virtual aperture will eventually reach the entrance slit. The system behaves like a pinhole camera, giving an infinite depth of field at the cost of some loss of resolution. The entrance slit is mapped to a $70 \mathrm{~cm}$ line at the center of the vessel while the resolution is about $1 \mathrm{~cm}$ at that same location and was judged acceptable. With this optimized set up, using 15 tracks of 20 pixel height each, about $35 \%$ of the OMA head as well as the entire grating could be illuminated.

Finally, an adjustable mirror was positioned on the focal point of L2 allowing to scan the entire plasma chamber as shown again in Fig. 4.8. At angles for which the light grazes the mirror, the corresponding track data were discarded. The mirror could also be removed from the field of view allowing 
the central portion of the midplane to be seen.

\subsubsection{Calibration of the Optics}

By ray tracing it is possible to calculate the mapping of the inside of the vessel to the head of the OMA, assuming paraxial rays and thin lenses. A careful calibration of the system indicated that even though the lens L3 is used at wide angles, the relationship between image height and object height is still linear, as shown in Fig. 4.9. Corrections have to be made for the change of index of refraction of the lenses with wavelength. Knowing the exact position of objects (ports for instance) inside the vessel it is possible to check the mapping and make corrections to match the exact focal length of the lenses.

A rough optical alignment is performed as follows. The lenses are placed at the positions given by the ray tracing code taking into account the dependence of the focal point on the wavelength. With the OMA turned off, a laser and a splitting cube are used to align the first lens with the slit and the back mirror of the spectrometer. Still using the laser, the optical axis is aligned perpendicular to the machine.

The final alignment is done as follows. The lenses' positions are adjusted to have a line near the wavelength of interest well in focus. A mercury pen lamp was used for this purpose. The spectrometer is then put in zero order and the OMA turned on in continuous mode. First it is checked that the optical axis is perpendicular to the machine by viewing the image given by the OMA on a monitor, adjustments are made such that the center of the port coincides with 

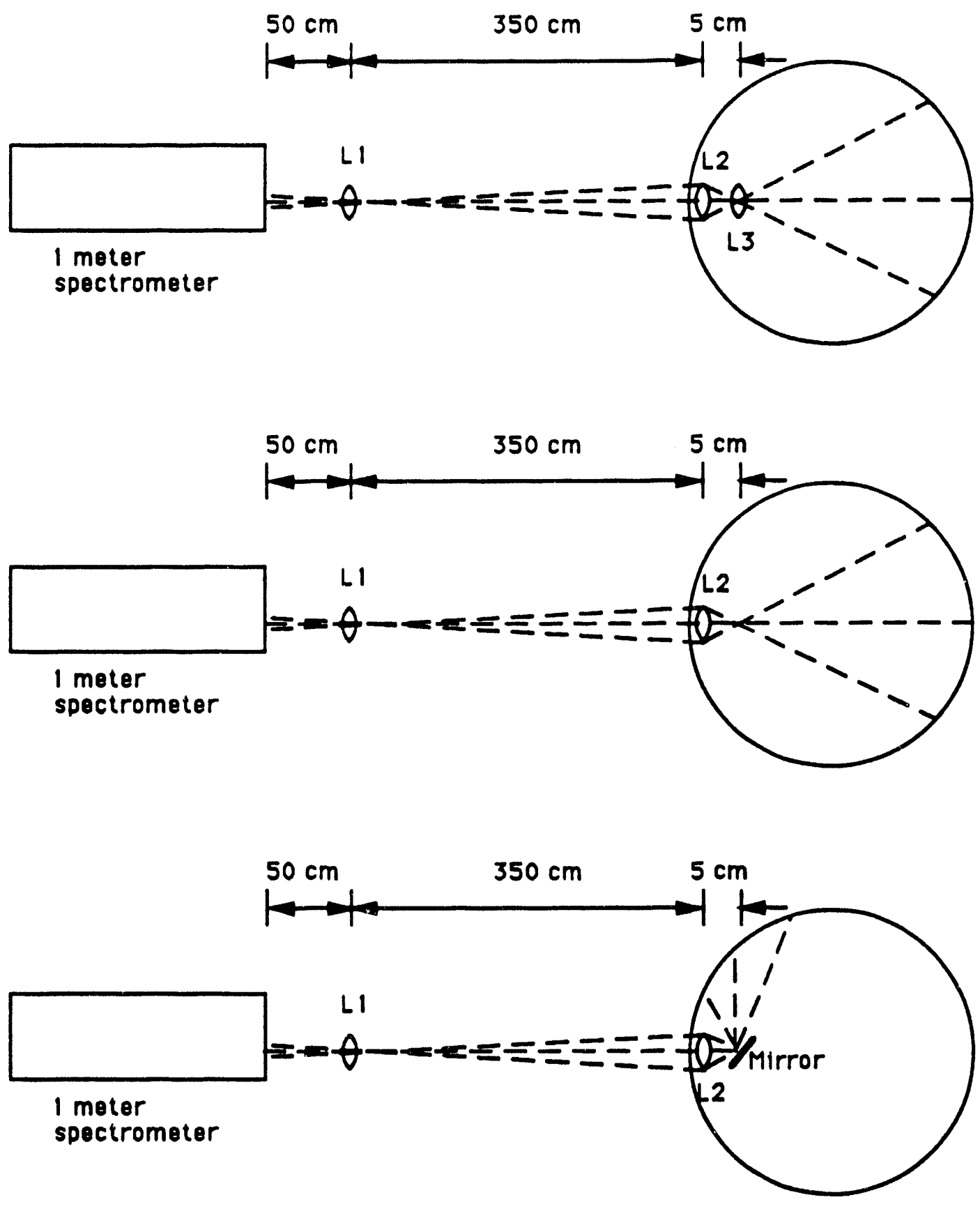

Figure 4.8: Wide angle lens optical system. Top: from ray tracing. Middle: actual system without rotating mirror. Bottom: actual system with rotating mirror. 


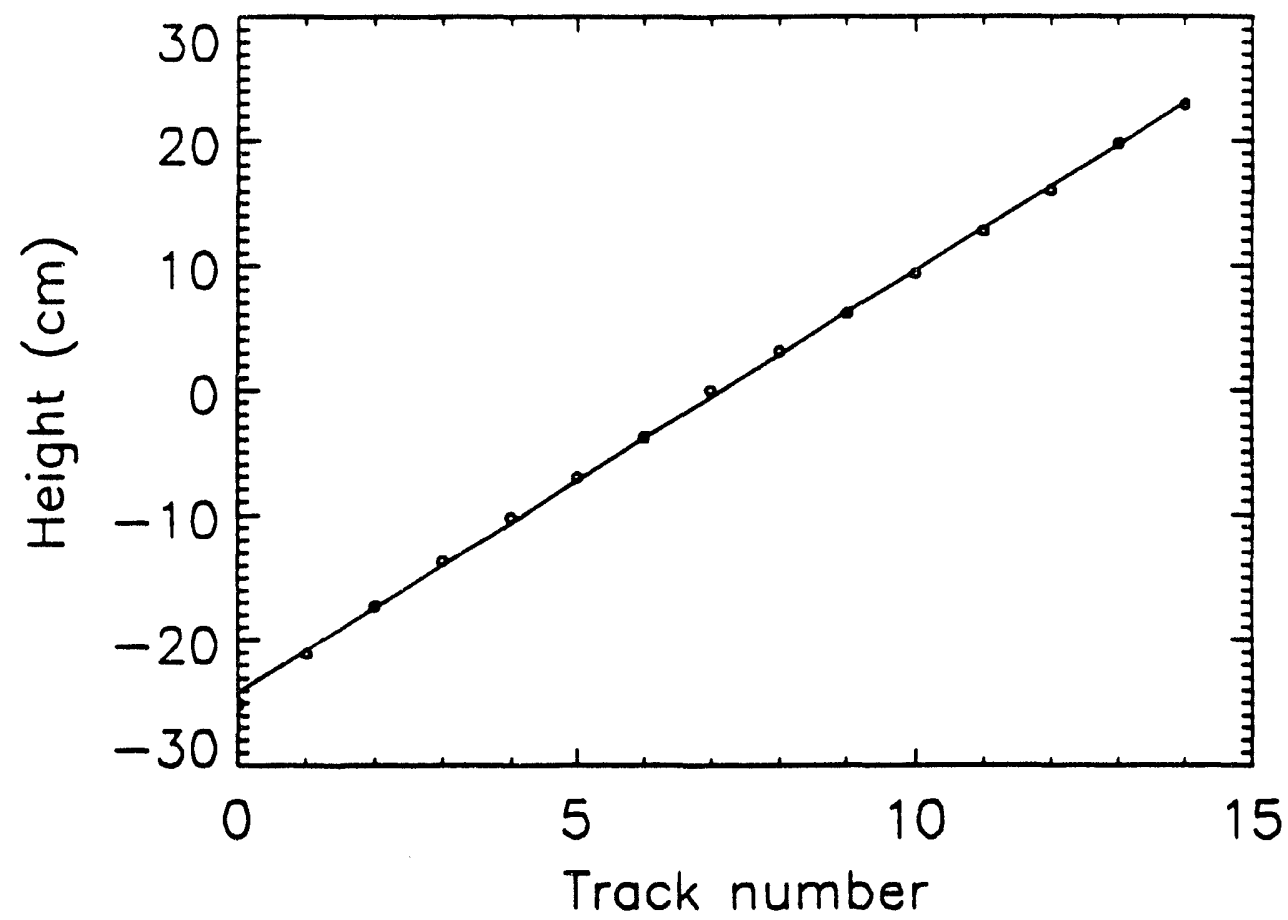

Figure 4.9: Calibration of the wide lens for an object placed at 1 meter.

the opposite port inside the machine.

The inner mirror allows for scanning of the plasma at different chord parameters. It has two degrees of freedom: us and down motion to align its center with the optical axis, and rotation to scan at different angles. The mirror is rotated until an image is observed that is symmetric to the case when there is no mirror. The optical axis is then grazing the mirror and this defines $\theta=0$ for the second degree of freedom. This alignment gave a $1^{\circ}$ precision in determining the angle of the final optical rays coming from the vessel.

A measure of the sensitivity in intensity of this optical system as a function 
of object position. indicated less than $10 \%$ variation. As explained in the next section, by overlapping the viewing regions when doing a scan in chord parameter, these variations are effectively averaged out. It was verified that the mirror reflectivity does not change as function of reflection angle nor as a function of time due to plasma deposition on its surface.

Because the OMA head uses electron beams to intensify the signal and to read the target, like a TV screen it is sensitive to magnetic field. To minimize the effect of the bias magnetic field on the OMA, it was enclosed in 3 concentric mu-metal cylinders. Mu-metal has a large $\mu$ and tends to exclude any DC magnetic field [31]. Despite this precaution, the magnetic field still has a small effect on the detector. The entire image appears rotated by about $8^{\circ}$ on the detector head. Experimentally there are 3 consequences due to that small rotation:

- The instrumental width is increased from 4.5 to 5.5 channels when using tracks of 20 pixels height. This can be subtracted in quadrature from the measured line widths. The measured line widths vary from 7 to over 20 channels. For tracks 50 pixels high, the instrumental width would be increased to about 9 channels and could introduce a large experimental error in estimating the plasma line widths.

- Each track now shows an appreciable wavelength shift compared to the non rotated image. This is taken care of by taking a wavelength calibration with the bias field turned on.

- Finally, because each line is shifted vertically, the track to angle calibration has to be done also with the bias field turned on. For example if 
track \#8 corresponds to the optical axis without the bias field. when it is turned on, now track \#9 could correspond to the optical axis.

\subsubsection{Relative wavelength calibration}

We added a small mirror of $1 / 2 \mathrm{~cm}^{2}$ area located at the center of the grating and attached to its mount, the grating area being about $100 \mathrm{~cm}^{2}$. The mirror will image the entrance slit of the spectrometer for all wavelengths on the detector head. The result is a reference line superimposed on the other spectroscopic lines. We can deduce from it any apparent wavelength shift due to microscopic motion of the internal parts of the spectrometer. Thermal expansion and contraction, and vibrations will afiect the wavelength calibration over several hours. It is then possible to estimate within one channel if a measured spectral line shift between two different shots is real or not. This reference line also gives a shot to shot comparison of the total intensity emitted in the line of sight for the wavelength range of sensitivity of the OMA.

\subsection{Line Broadening Mechanisms}

For a given atomic transition, each ion emits at the same specific wavelength in its own frame of reference. There will be a natural line width due to the finite lifetime of the upper state [32].

At high enough densities, the electric field due to the surrounding particles 
causes a measurable Stark broadening of some lines [34]. The line emission as a function of wavelength is usually a Lorentzian.

At high enough ion temperatures, a broadening will also occur due to the Doppler effect [34]. An ensemble of particles characterized by a temperature $\mathrm{T}$ will have a Maxwell-Boltzmann distribution in velocity space:

$$
P(v)=\left(4 / \pi^{1 / 2}\right)(m / 2 k T)^{3 / 2} v^{2} e^{\left(-m v^{2} / 2 k T\right)} .
$$

The observed wavelength from each atom is Doppler shifted in wavelength according to the line of sight velocity. If we take $z$ to be the line of sight axis then

$$
P\left(v_{z}\right)=\pi^{-1 / 2}(m / 2 k T) e^{\left(-m v_{z}^{2} / 2 k T\right)}
$$

The distribution is now reduced to a Gaussian.

The resultant line profile is then the sum of all the shifted profiles weighted with the velocity distribution. In general the resultant profile is the convolution of a Gaussian and a Lorentzian and is called a Voigt profile. For the case where the Lorentzian width is negligible compared to the Gaussian width, then the resultant profile is a Gaussian centered on the unshifted line with full width at half maximum (FWHM) in wavelength related directly to the temperature by

$$
\frac{\delta \lambda}{\lambda}=7.7 \times 10^{-5}\left(\frac{T}{m / m_{p}}\right)^{1 / 2}
$$

where $m_{p}$ is the proton mass and $\mathrm{T}$ is the temperature in $\mathrm{eV}$.

In MS as in most hot plasma experiments, the natural line width is negligi- 
ble for all species. In MS. the Stark broadening mechanism is important for the low mass ions like hydrogen. deuterium and marginally for helium. For oxygen and carbon, the Stark broadening effect is estimated to be much smaller than the instrumental width of the profile while the temperature broadening is equal to or larger than the instrumental width.

If the plasma has a bulk velocity, the line will appear shifted.

$$
\frac{\delta \lambda}{\lambda}=\frac{V_{z}}{c}
$$

where $V_{z}$ is the directed motion fluid velocity and $\mathrm{c}$ is the speed of light.

This is the case when rotation is present [35]. If two or more portions of the plasma move at different velocities along the same line of sight, the resultant profile will be the sum of two or more Gaussians shifted from each other. A sum of different cells having different velocities along the same line of sight can give an apparent temperature when measuring the line width. This is called motional broadening. It is therefore important to verify that the measured line profile is actually Gaussian. 


\section{Chapter 5}

\section{Results}

\subsection{General observations}

Assuming that the measured line width gives average ion temperature over the line of sight, measurements of the line averaged temperatures were taken for OII, OIII, OIV, OV, CIII and CIV. It became apparent that the ion temperature was higher than the electron temperature estimated from the presence of the highest ionization stages of carbon and oxygen.

The temperature is highest for the highest ionization stages. Paraxial and radial lines of sights were used. The paraxial measurements have shown under certain conditions non Gaussian profiles [36], indicating bulk motion of the ions. The ion temperature is dependent on the particular experimental 
conditions. The following generalizations could be made:

- The ion temperature scales inversely with the puffed gas pressure.

- The ion temperature appeared higher with a fast $I_{z}$ rise time $(30 \mu \mathrm{s})$ than with a slower rise time of $(75 \mu s)$.

- The ion temperature as well as the electron temperature were increased with the addition of two glass plate liners around the reversal coils forcing the current to flow along the central magnetic fields and therefore doubling the current in that region.

- It is found that the ion temperature is highest at early times and decays, approaching the electron temperature as time evolves.

\subsection{Processing of data}

Figure 5.1 shows the ion temperature obtained for different oxygen stages when looking from the end, $15 \mathrm{~cm}$ off the vacuum chamber symmetry axis. The experiment was operated with Ta covered aluminum electrodes and with stainless steal cones to stabilize the plasma.

In order to obtain an ion temperature, the line profile was fitted to a Gaussian plus a background with constant slope,

$$
f(X)=A+B X+C e^{-X^{2} / d^{2}}
$$



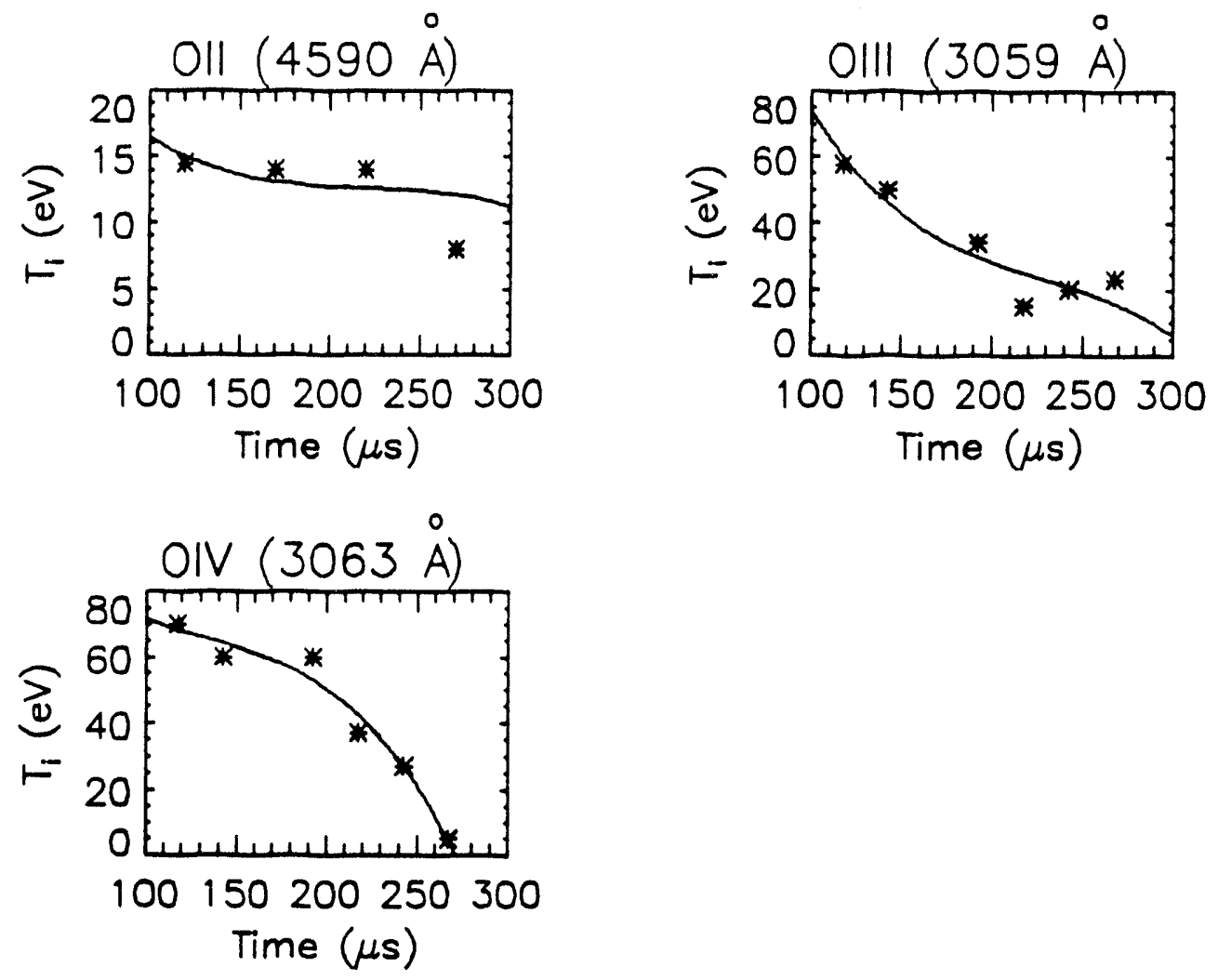

Figure 5.1: Ion temperature of different oxygen stages as a function of time. One temporal data point is taken per shot.

where $\mathrm{X}$ is the channel number. It is then transformed into wavelength by calibrating the instrument, usually with a thorium lamp. The parameters are found by a least square fit routine, using the Interactive Data Language (IDL). Figure 5.2 shows a sample of an experimental line shape and its fit. The standard deviation on the line width from the fit to a Gaussian is about $2 \%$ of the width, which is a much smaller error than the shot to shot variations (> $10 \%)$. The corresponding standard deviation on the temperature is therefore about $4 \%$. 


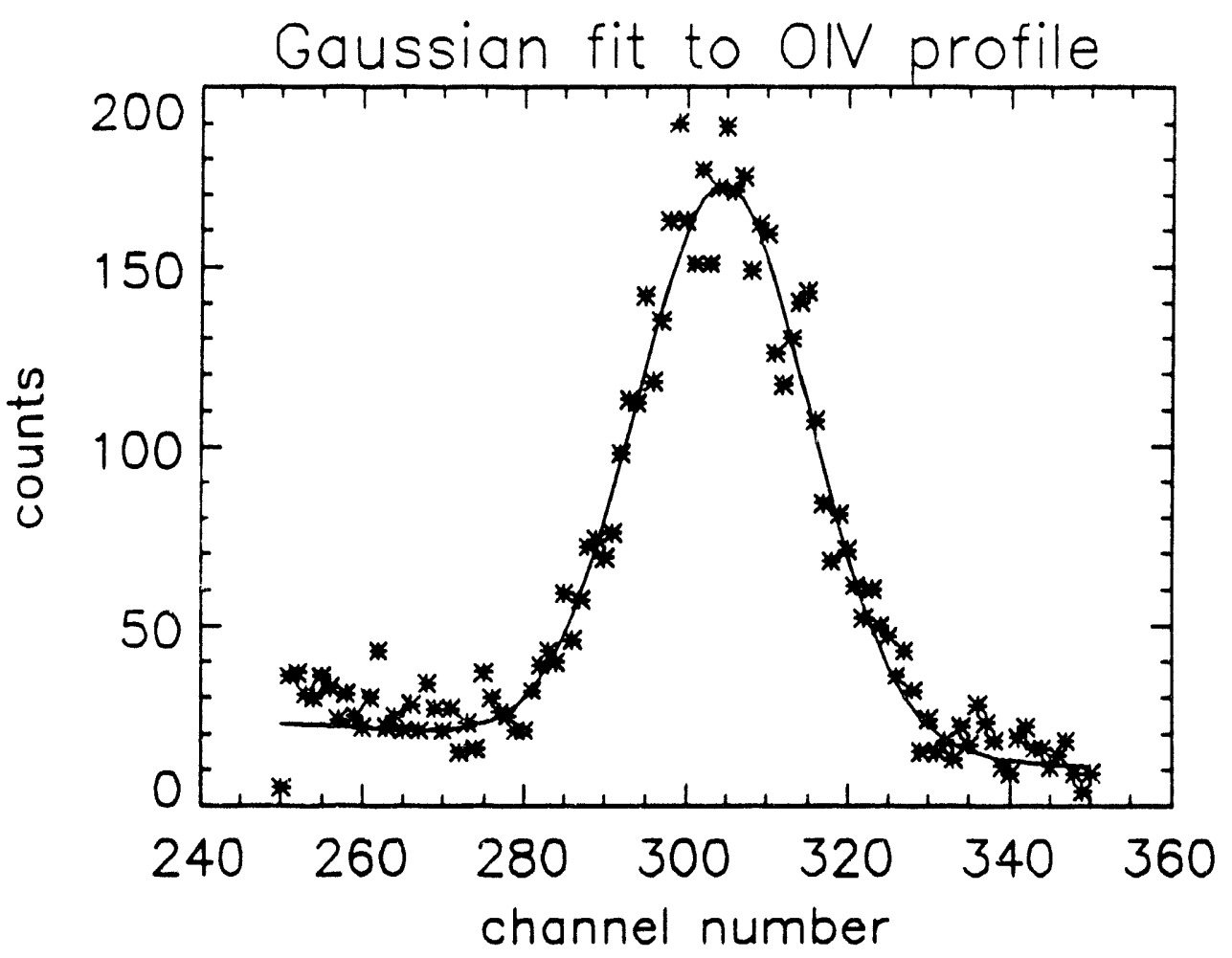

Figure 5.2: Typical fit of an OIV experimental line shape to a Gaussian plus constant slope background.

The instrumental width is subtracted in quadrature from the data to obtain the plasma line width:

$$
W_{\text {obs }}^{2}=W_{\text {line }}^{2}+W_{\text {instr }}^{2}
$$

Although, ideally, the instrumental function of the spectrometer is a rectangular function of wavelength, because of cross-talk between the channels on the OMA head, and because of geometrical and wave optics error, the actual experimental line shape is rounded and can be approximated by a Gaussian. For a slit of less than $20 \mu \mathrm{m}$, the instrumental width is 3.5 channels. For the $100 \mu \mathrm{m}$ slit used, the instrumental width is increased to 4.5 channels. 
When including the effect of the bias magnetic field, a 5.5 channel instrumental width is obtained. In the case of the helium line at $4686 \AA$. because it is a fine-structure multiplet, the effective natural line width is non-negligible (.5 $\AA$ [White]) and is also subtracted in quadrature.

\subsection{Single shot time resolved results}

The use of a rotating slit allowed us to obtain a time history in fewer shots as described in the previous chapter. Five or six line profiles could be obtained for each shot.

\subsubsection{Ion temperature and line intensity}

Figure 5.3 shows the temperature and intensity time dependence for different oxygen and carbon ions using this technique. For these shots the electrodes are Elkonite and the reversal coils have been wrapped with teflon and Titanium layers of tape. Figure-eight coils stabilize the plasma. The line of sight is a chord at $12 \mathrm{~cm}$ off the mid-plane. The total intensity of the line is determined from the area of the fitted Gaussian profile. In principle, a time dependence

could have been obtained for different chord parameters in this fashion, allowing to invert for the line profile as a function of radius. However, because of the random timing of the wheel, the possibility of its angular velocity changing from shot to shot and the fact that each track is then looking at a slightly 

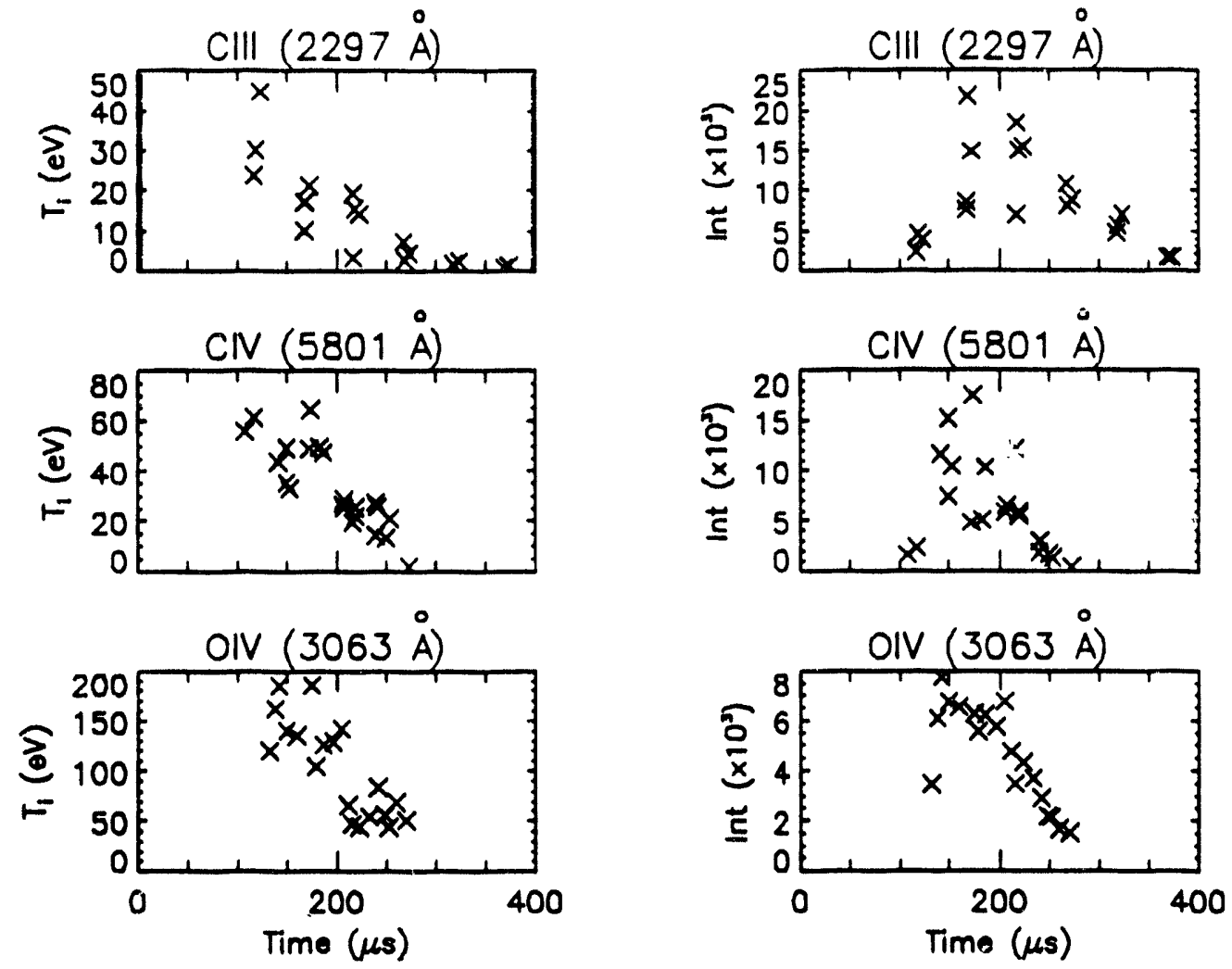

Figure 5.3: Ion temperature and intensity of different impurities measured using a rotating slit. Five or six data points are obtained per shot fired.

different region of the plasma, a different method was chosen to perform a spatial scan.

\subsubsection{Impurities}

The high resolution data described up to now, were taken in second, third or fourth order with a 1200 line/mm grating blazed for $10,000 \AA$. About 80 $\AA$ could be seen in one OMA frame. We replaced the dispersing element 
of the spectrometer by a 150 line/mm grating blazed at $5000 \AA$ in order to observe over a wider spectral range. About $600 \AA$ of spectrum per frame could be obtained. In this low resolution mode. it was possible to obtain a time resolved spectrum of the 2200 to $5400 \AA$ range in a few shots with the rotating slit. It is a similar spectrum to the one obtained with the linear OMA. Most impurity lines could be identified as shown in Fig. 5.4. As discussed earlier, the most frequently occuring lines are from carbon, oxygen and aluminum. At lower intensities we observe copper lines, which could come from the figureeight coils and also from the electrodes. After wrapping the reversal coil with teflon, some fluorine lines appeared. The next section describes the behavior of the $H_{\beta}$ and $D_{\beta}$ lines when using the high resolution grating.

\subsection{3 $H_{\beta}$ and $D_{\beta}$ data}

In addition, we made a time and space resolved scan of the $H_{\beta}(4860.0 \AA)$ and $D_{\beta}(4861.33 \AA)$ lines. $D_{\beta}$ is the main species line. To our surprise we could see the deuterium ions being replaced by hydrogen ions as a function of time as shown in Fig. 5.5. This indicates that an important particle transport mechanism takes place, shortening the particle confinement time [22]. These results might suggest that covering the walls by titanium gettering should solve many of the impurity problems. However, the only significant difference observed when gettering, was a faster pumping of the main species $\left(D_{2}\right)$ during the discharge without much effect on hydrogen, see Fig. 5.6. Oxygen and carbon impurities did not seem affected either by gettering, suggesting that they have their source at a location that is not covered by titanium or where 

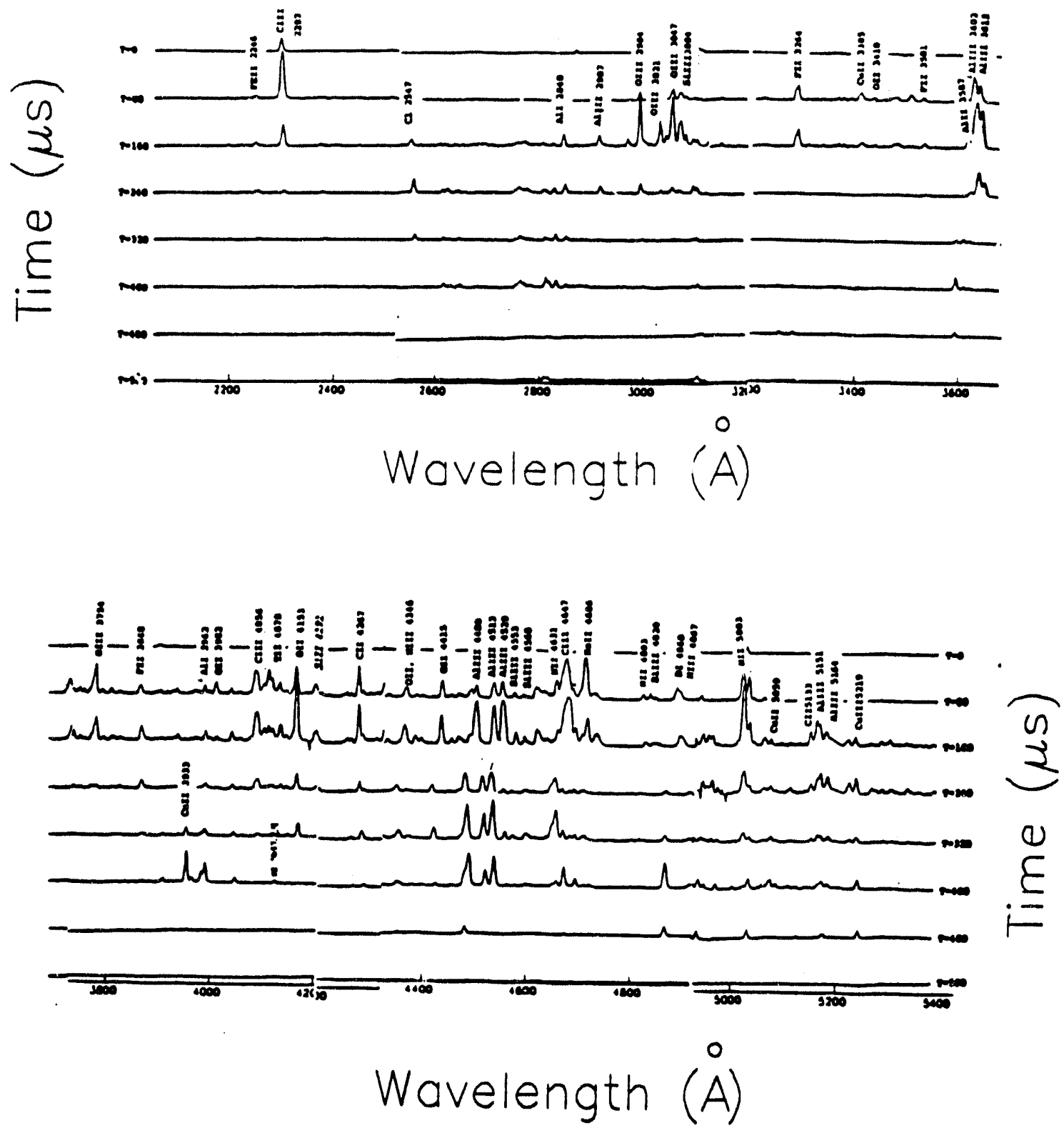

Figure 5.4: Spectrum of impurities as a function of wavelength and time from 2200 to $5400 \AA$ 


\section{$H_{\beta}$ and $D_{\beta}$ PROFILES before gettering}
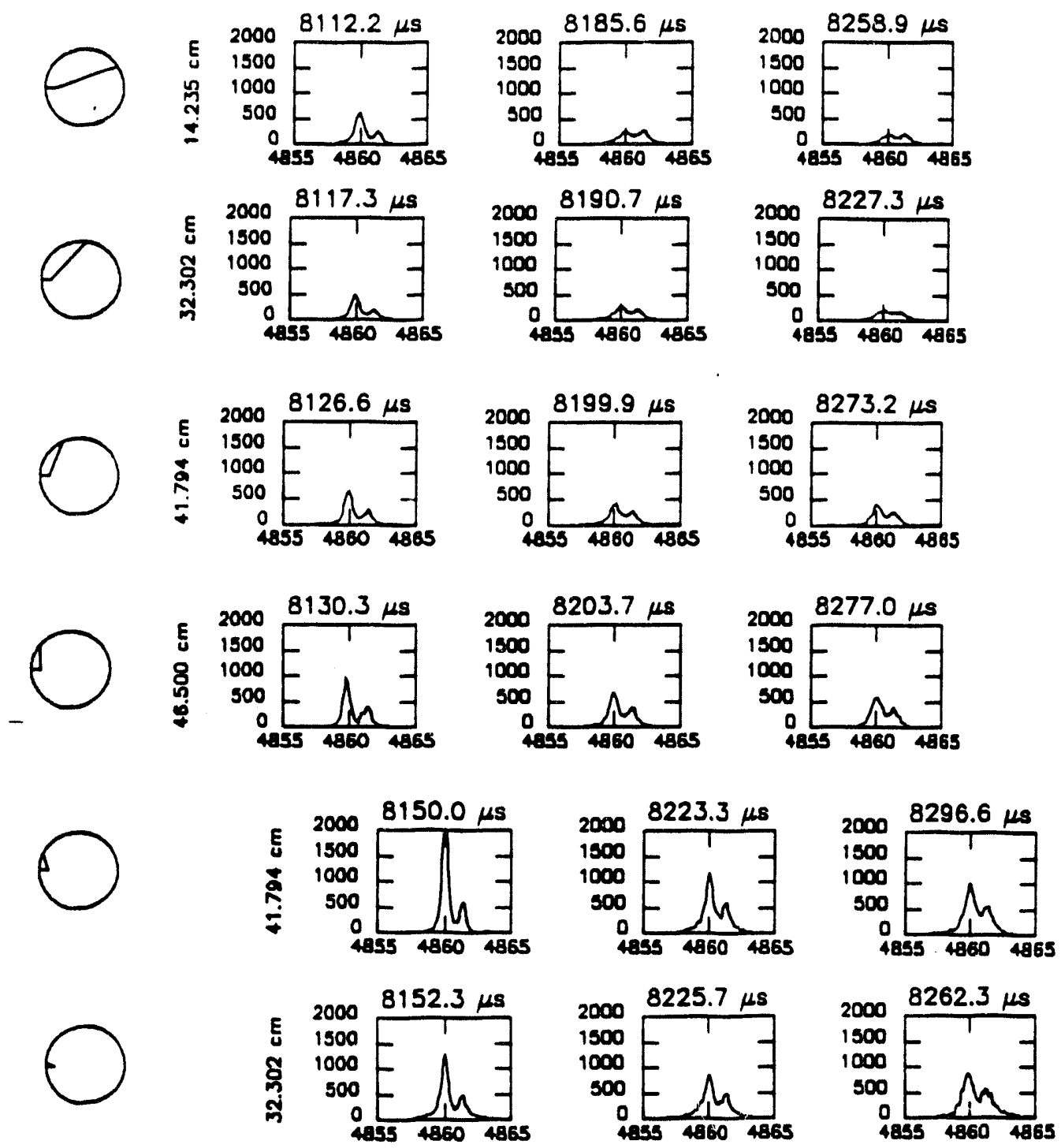

Figure 5.5: $H_{\beta}$ and $D_{\beta}$ spectra for different chords and tir..es before performing gettering 


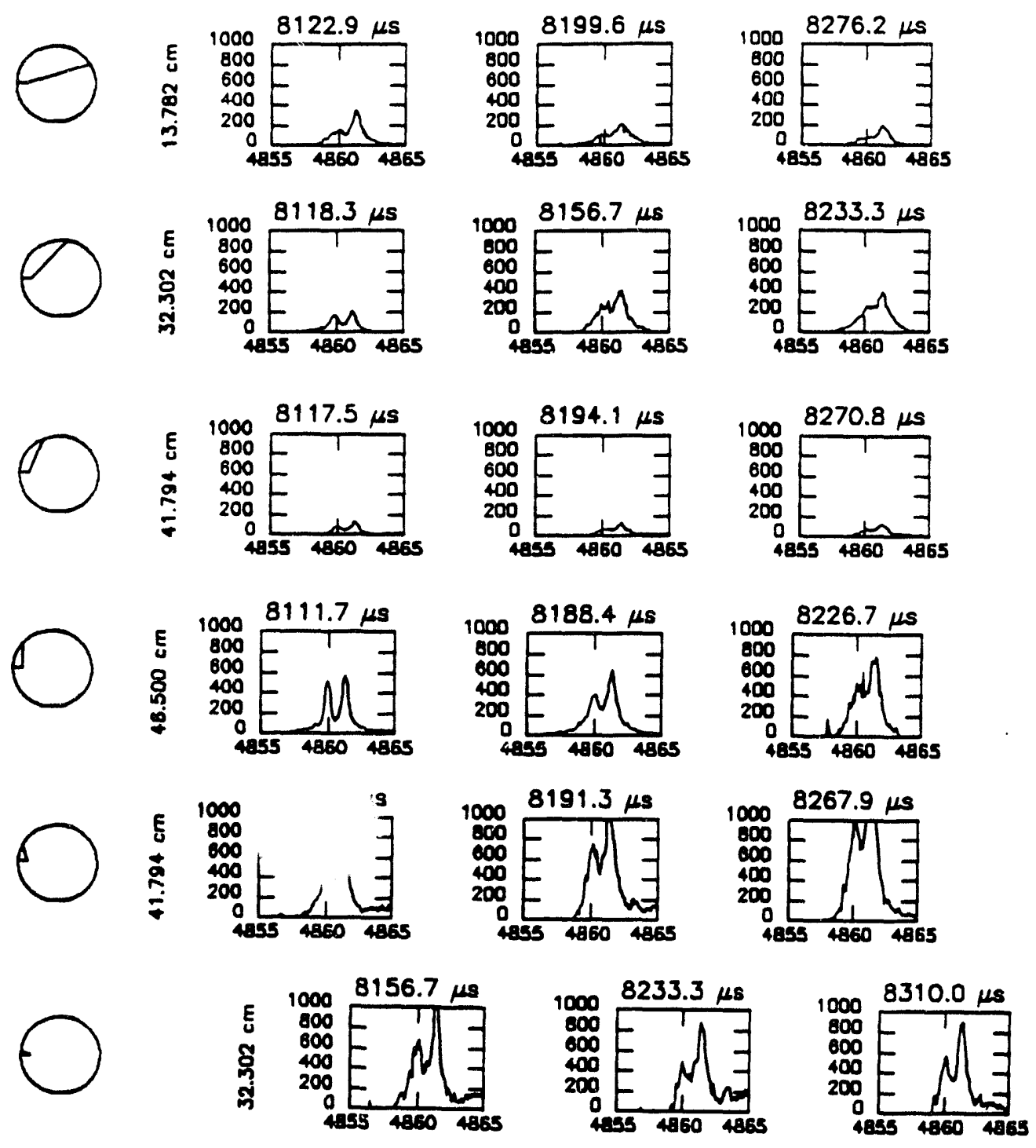

Figure 5.6: $H_{\beta}$ and $D_{\beta}$ spectra for different chords and times after performing gettering 
the titanium is eroded quickly during the discharge.

\subsection{Single shot space resolved results}

For the data reported here, the wide angle lens system described in section 4.4.4 was used. The OMA was gated so that a single time interval of $20 \mu \mathrm{s}$ is obtained on each shot. The line profile obtained in a line of sight represents the sum of all the line profiles emitted from the different regions of the plasma intercepting the viewing chord. By taking data from a sufficient number of chords it is possible to invert the data and recover the line profile from each region of the plasma. Considerable simplifications occur if we assume axisymmetry. The inversion is reduced to calculating an integral.

Obtaining accurate results with the Abel inversion requires an extensive array of data from the axis to the edge. Because we had only a sparse array of data, the least square fit method described in the next section was used instead. It had the advantage of giving smaller error bars on inverted data near $r=0$. The total line intensity can be inverted easily with this method. We can also invert the line emission as a function of wavelength to recover the emitting line profile as a function of radius, from which we can now extract the temperature as a function of radius.

When one considers radial and toroidal mass motions of the plasma, one can not assume axisymmetry since we have to consider the component of the shift along the line of sight which gives a $\sin \phi$ or a $\cos \phi$ term in the shift of 
the emitting profile. The inversion for the total intensity is still unchanged but the inversion by wavelength is now inaccurate and may attribute larger temperatures than really exist to the mass motion regions. A least square fit including those new parameters could resolve the problem. The presence of toroidal rotation is easier to detect than radial motion because it gives a net shift to the observed lines.

If the plasma was not optically thin for the observed profile, then again axisymmetry is not a valid assumption and a least square fit method is needed. In the present work, the observed lines are not resonance lines and no reabsorption should take place for our plasma parameters. Magnetic diagnostics indicate a pinch of the plasma at early times giving radial velocities of less than $1 \mathrm{~cm} / \mu \mathrm{s}$ which is below the resolution of the instrument and therefore radial mass motion was not considered. A line of sight plasma shift was observed for a narrow set of chord parameters at early times. In the present study, the line shift was not taken care of in the inversion and as a consequence, for those particular radii the inverted temperature is larger than it should be.

\subsubsection{Inversion method}

The inversion is performed with the raw data and then repeated several times with data that consists of the original data plus random noise with standard deviation equal to the actual standard deviation in the intensity and temperature data. When inversions are superimposed, this gives an idea of the actual error in the inversion. 
Here follows a description of the least square fit technique used. Cylindrical symmetry is assumed: $e=e(r)$, the atomic emission per unit volume is a function of radius but not of toroidal angle. A line of sight at a given chord parameter intercepts different radii of emission as shown in Fig. 5.7. The observed intensity $I$, is related to the emission by the following formula, using matrix notation:

$$
(I)=(M)(e)
$$

$M$ is an operator that integrates analytically the radial emissions for a given chord parameter. To first order, the plasma is separated into several shells of different radii. The emission, e, is assumed to change linearly from one shell to the other, as shown in Fig. 5.8.

$I$ is the resultant line of sight intensity and is obtained from measurements. The inversion method then finds the best values of $e$ such that the least square fit of $(M)(e)$ to $I$ is obtained. At a given time $t$, from $I(h)$ we obtain $e(r)$, where $h$ is the chord parameter. When we take into account temperature, the emission $e$ and the intensity $I$ become functions of wavelength. We can then invert for each wavelength: from $I\left(h, \lambda_{i}\right)$ we obtain $e\left(r, \lambda_{i}\right)$.

The observed line of sight wavelength profiles are well approximated by Gaussian functions of wavelength. Each Gaussian can be characterized by an intensity and by a full width at half maximum (FWHM) which can be related to an ion temperature. In order to average and smooth the data, the obtained line of sight intensities and linewidths are fitted to polynomial functions of chord parameter. Even order polynomials are chosen to ensure symmetry around $\mathrm{r}=0$. 


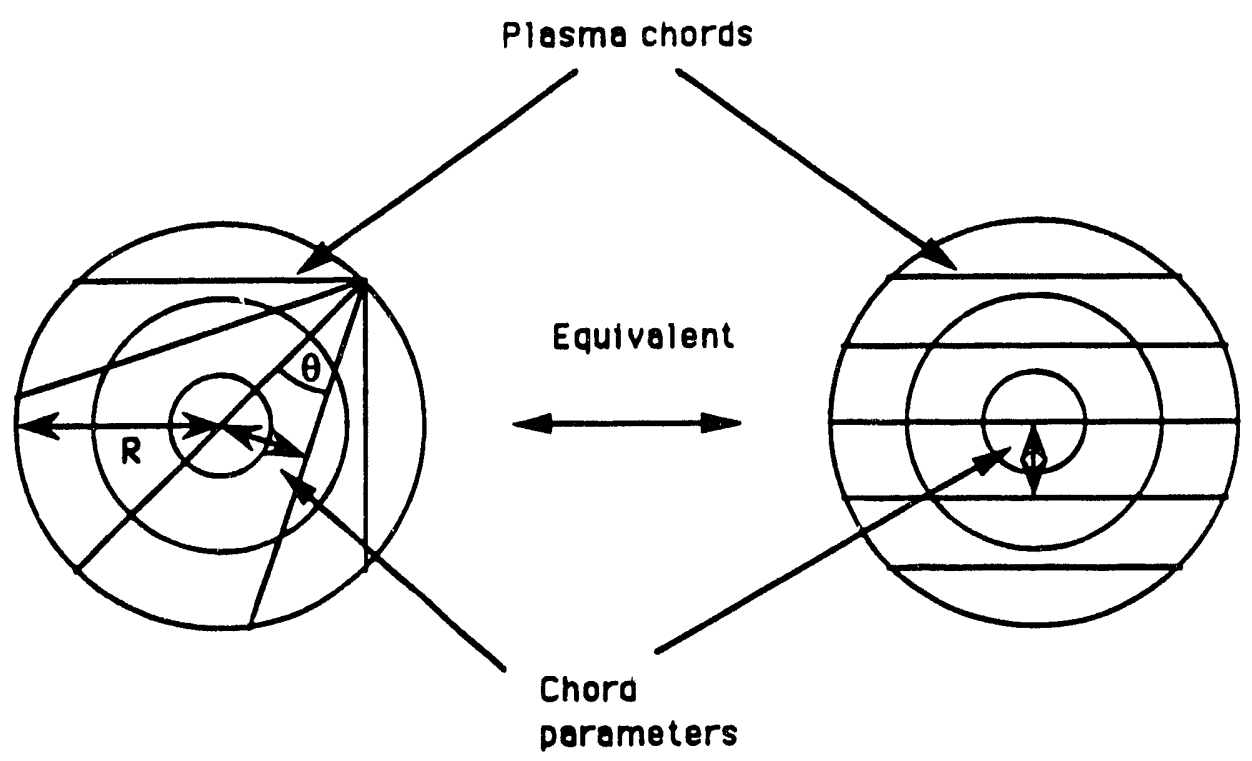

Figure 5.7: Diagrams showing how chords intercept different radii for two equivalent geometries.

Inversion is performed at about 10 evenly spaced wavelengths covering the entire profile. This inversion gives the emissivity as a function of wavelength, radius and time for a given line. The inverted profile is fitted to a Gaussian and finally the emission and temperature as a function of radius can be extracted.

Inversion can also be performed for the total line intensity directly. In that case we do not obtain temperature information. It was checked that there is agreement between the two methods. 


\subsubsection{Inversion of a test function}

Gaussian line profiles with specified intensities and widths at each radius are integrated along different chord parameters to simulate chord data. Each chord data is fitted to a Gaussian from which we extract the width and the intensity.

Figure 5.8 shows the temperature and intensity radial profiles chosen as test functions and their line of sight simulated values as a function of chord parameter.

The data are then inverted. The process was repeated several times, adding random fluctuations to the original data to simulate noise in experimental data. The noise added had $20 \%$ standard deviation in the intensities and in the line widths. Figure 5.9 shows the chord data plus noise and the corresponding inverted results. The spread in the inverted data gives an error estimate.

\subsubsection{OIV Chord data}

The wide angle lens has a field of view of $20^{\circ}$. Data are taken with and without the rotating mirror as shown in Fig. 4.8. The chord parameter $h$, is related to the scanning angle $\theta$ by

$$
h=R \sin \theta
$$



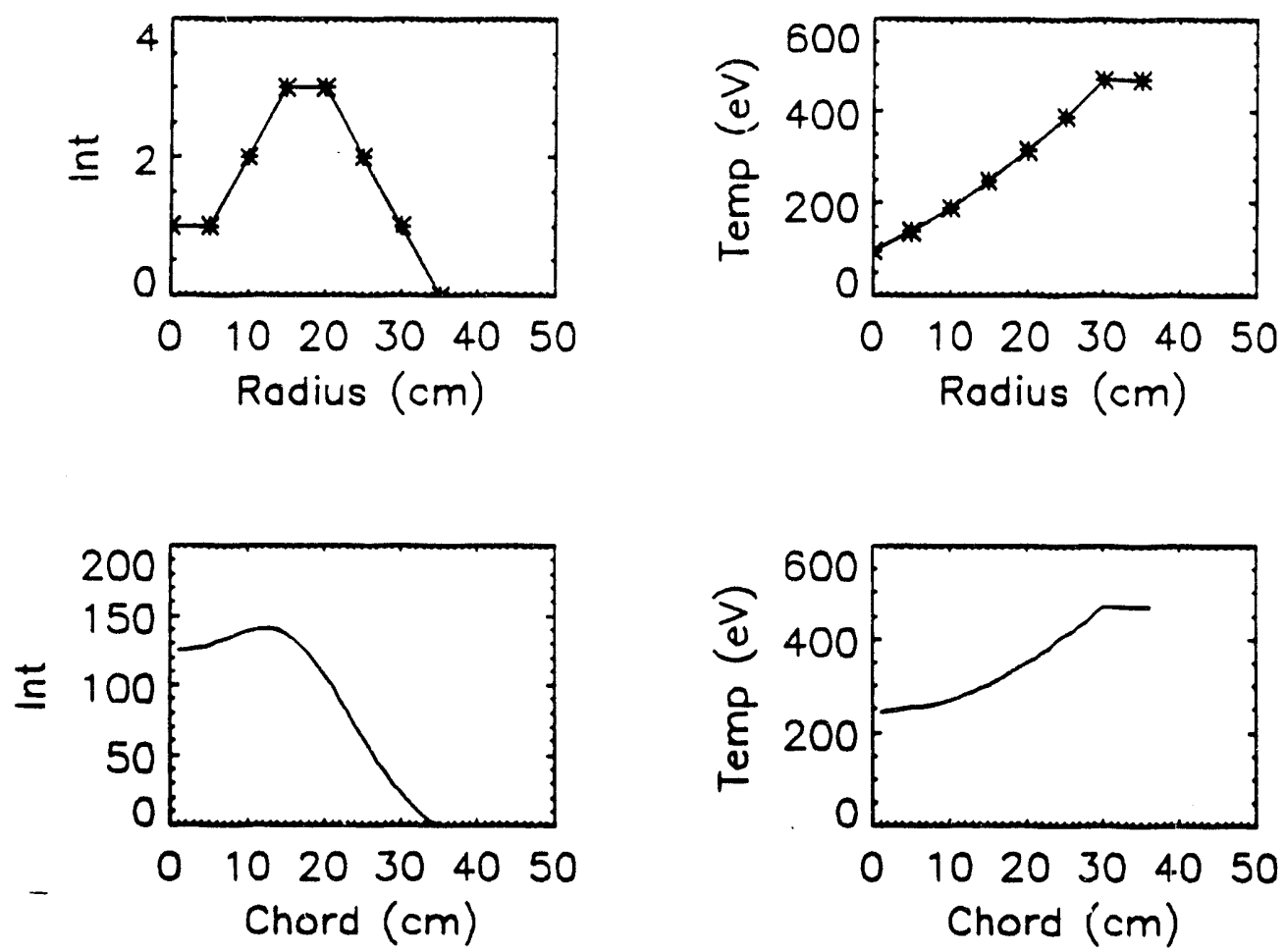

Figure 5.8: Top: temperature and intensity radial profiles used to test the inversion routine. Bottom: Simulated line of sight temperature and intensity from the integration of the test function.

The mirror angle is changed by $5^{\circ}$, giving a $10^{\circ}$ increment in scanning angle. This gives a $50 \%$ overlap in the scanned region from one mirror position to the other. Since the OIV species appears mainly at the center of the plasma (within a total viewing angle of $60^{\circ}$ ) only 7 mirror positions were necessary to perform a spatial scan. For each position the data are collected twice or more if the results were not reproducible or if the monitors showed excessive shot to shot variations. It is important to get the scan in as few shots as possible since plasma conditions could be slowly changing with time. This will 

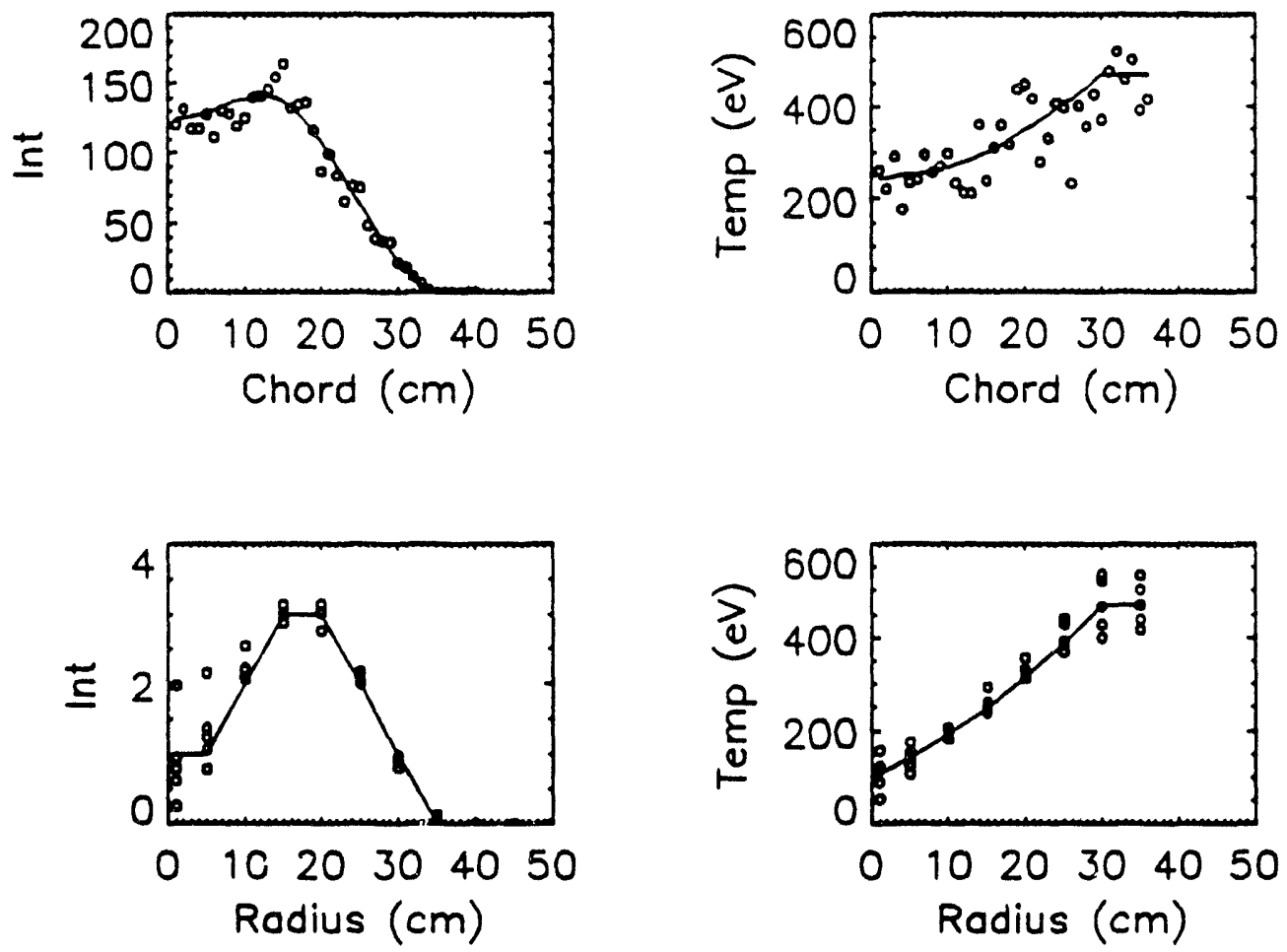

Figure 5.9: Top: Simulated chord data (solid line) and noise superimposed to the original simulated chord data (points). Bottom: Inversion of these test chord data. When no noise is added (solid line) the inversion gives back the original test function shown in the previous figure.

minimize the error when performing an inversion. The procedure is repeated for various times to get a time history of the line profiles. The tempera,ture and intensity of OIV $(3071 \AA)$ as a function of chord parameter is shown for different times in Figs. 5.11 to 5.15. The line of sight profiles are obtained by taking the observed profiles and subtracting the background signal. It is obtained by taking a shot without $I_{z}$ but with the bias field on because it has a small effect on the OMA head. The signal is treated in units of channel and is converted after inversion, into $\AA$ and then into $\mathrm{eV}$. 
Before performing the inversion. we can make the following observations:

- There is an up-down asymmetry in the data, in intensity and in temperature. Preliminary bolometry data also showed the same asymmetry in intensity, therefore making it less nrobable that the asymmetry is related to some systematic diagnostic error.

- For each time frame, the line intensity goes to zero for some chord parameter larger than about $35 \mathrm{~cm}$, indicating that after inversion of the data, the intensity should stay zero outside a radius equal to that chord parameter. Furthermore, because the intensity profile has a minimum at zero chord parameter, we can predict that the inverted profile will be hollow.

- The relative line shifts were also estimated, using a reference line as described in section 4.4.6. No significant line shifts were found, except for the chord parameters where the highest ion temperatures were found, around $r=20 \mathrm{~cm}$. This means that those temperatures may be the result of motional Doppler broadening.

- The main characteristics of the observed ion heating are high temperature $(500 \mathrm{eV})$ at early times decreasing exponentially with a $20 \mu s$ decay time until it reaches the electron temperature. The hot ions are observed only in the path of the $I_{z}$ current. This does not mean that there are no hot ions outside this volume. It only means that in this volume the electron temperature is high enough for the OIV and OV species to be observed. Observation of lower ionization stages always showed lower ion temperatures. However, if high temperature OIII was present at 
very small radius. its observation could be hidden by the superposition of lower OIII temperatures at larger radius.

\subsubsection{Results of the inversion}

The inversion was performed separately for the upper and lower half of the plasma. Figure 5.10 shows a typical inversion profile and its fit to a Gaussian. The fit is best for the large intensity regions. The standard deviation

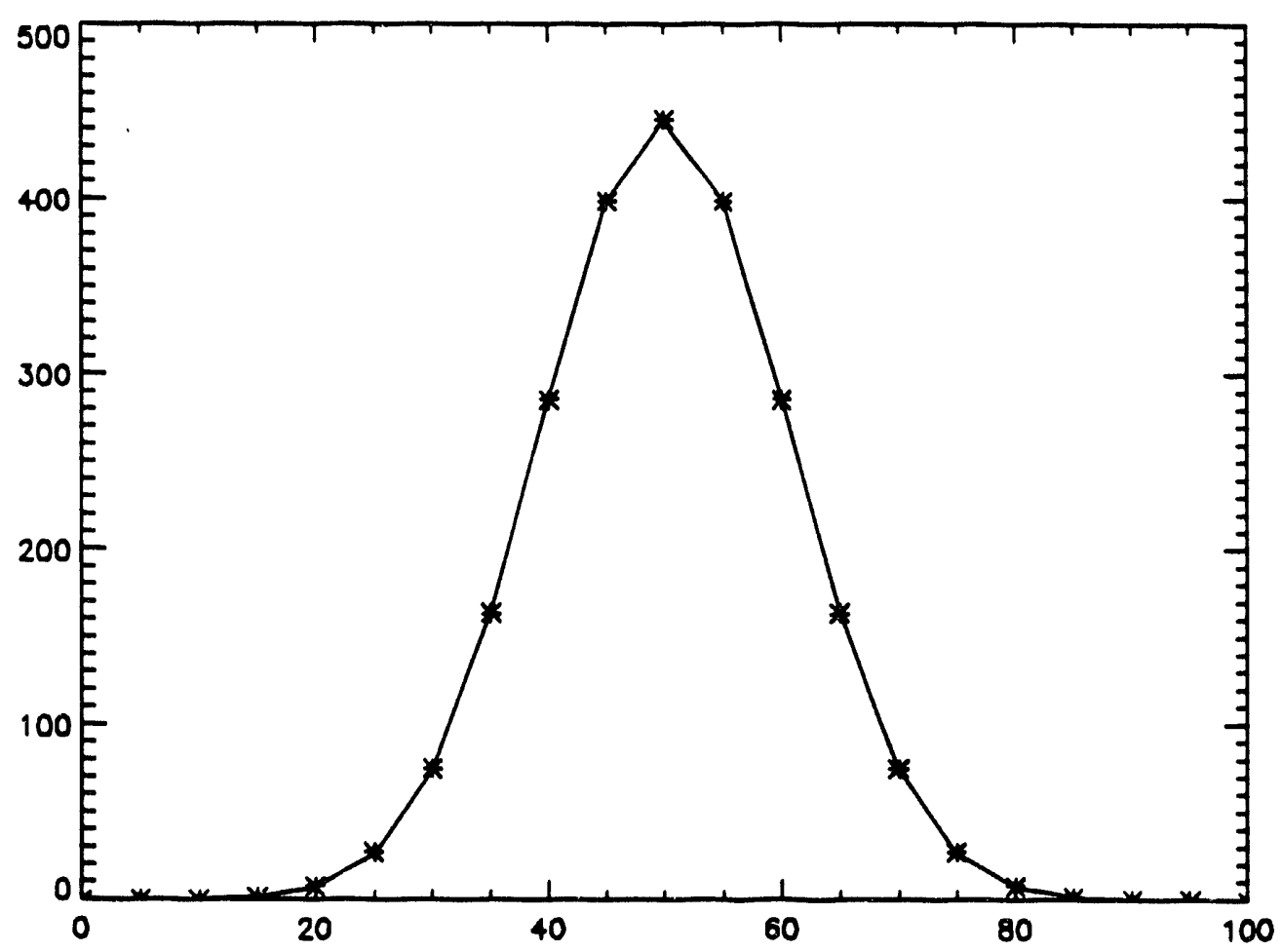

Figure 5.10: Typical inverted profile and its fit to a Gaussian.

in te $a_{c}$ erature from the fit is less than $1 \%$ of the temperature. This is much 
smaller than the propagation of error from the shot to shot fluctuations of the original data. A compromise has to be made between radial resolution and the error bar in the inverted signal. The inversion is repeated adding noise to the raw data equal to their standard deviations to propagate the error to the inverted results. The results are shown in Fig. 5.11 to 5.15. The general
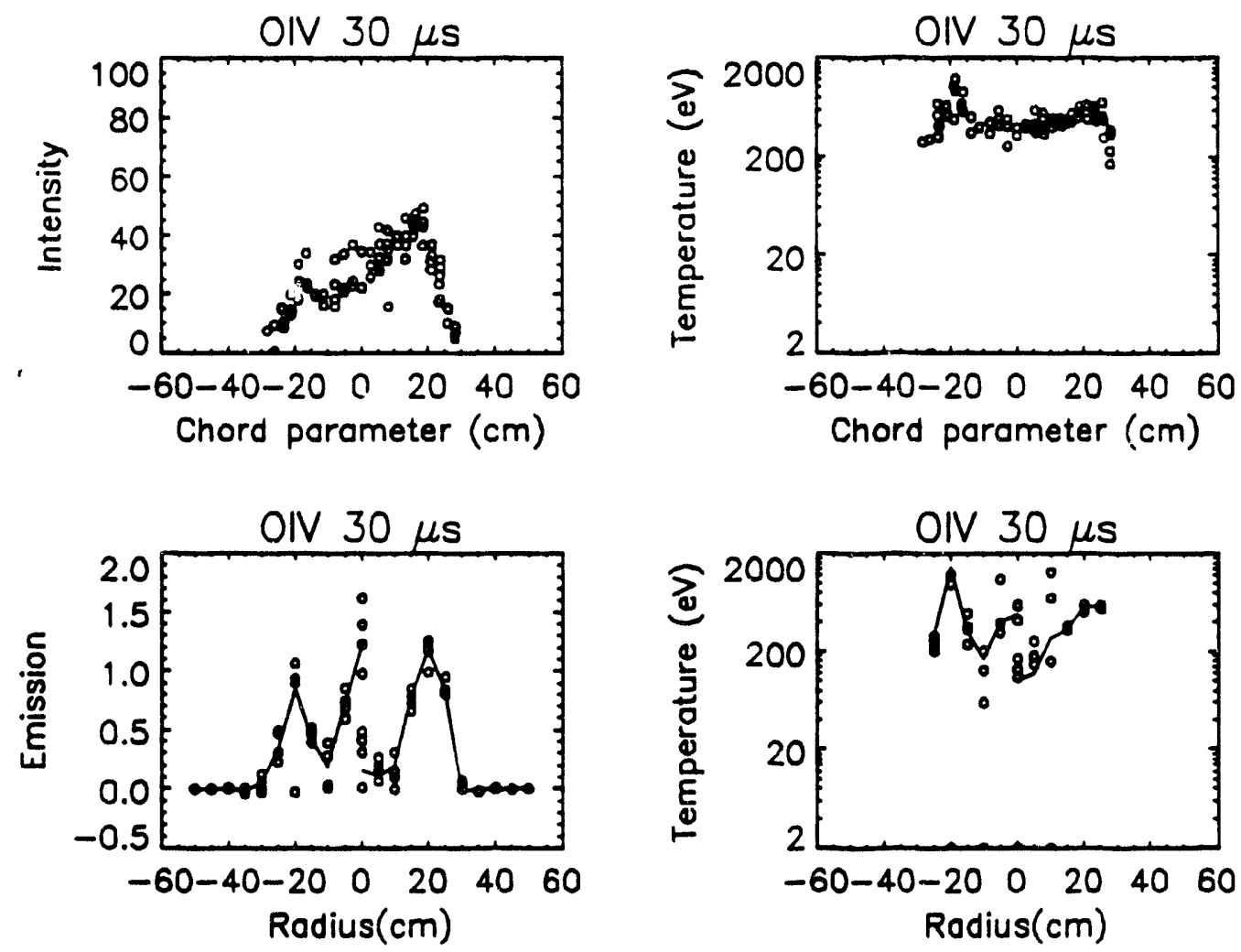

Figure 5.11: Top: Intensity and temperature line of sight measurements for OIV (3063 $\AA)$ at $30 \mu \mathrm{s}$. Bottom: The solid line is the inversion of these measurements. The circles are the inversion when noise is added to the data.

behavior of OIV radial emission was to form a ring that is slowly compressing with time. It appears that the radius of this ring in the mid-plane corresponds to the magnetic field line joining one electrode to the other. 

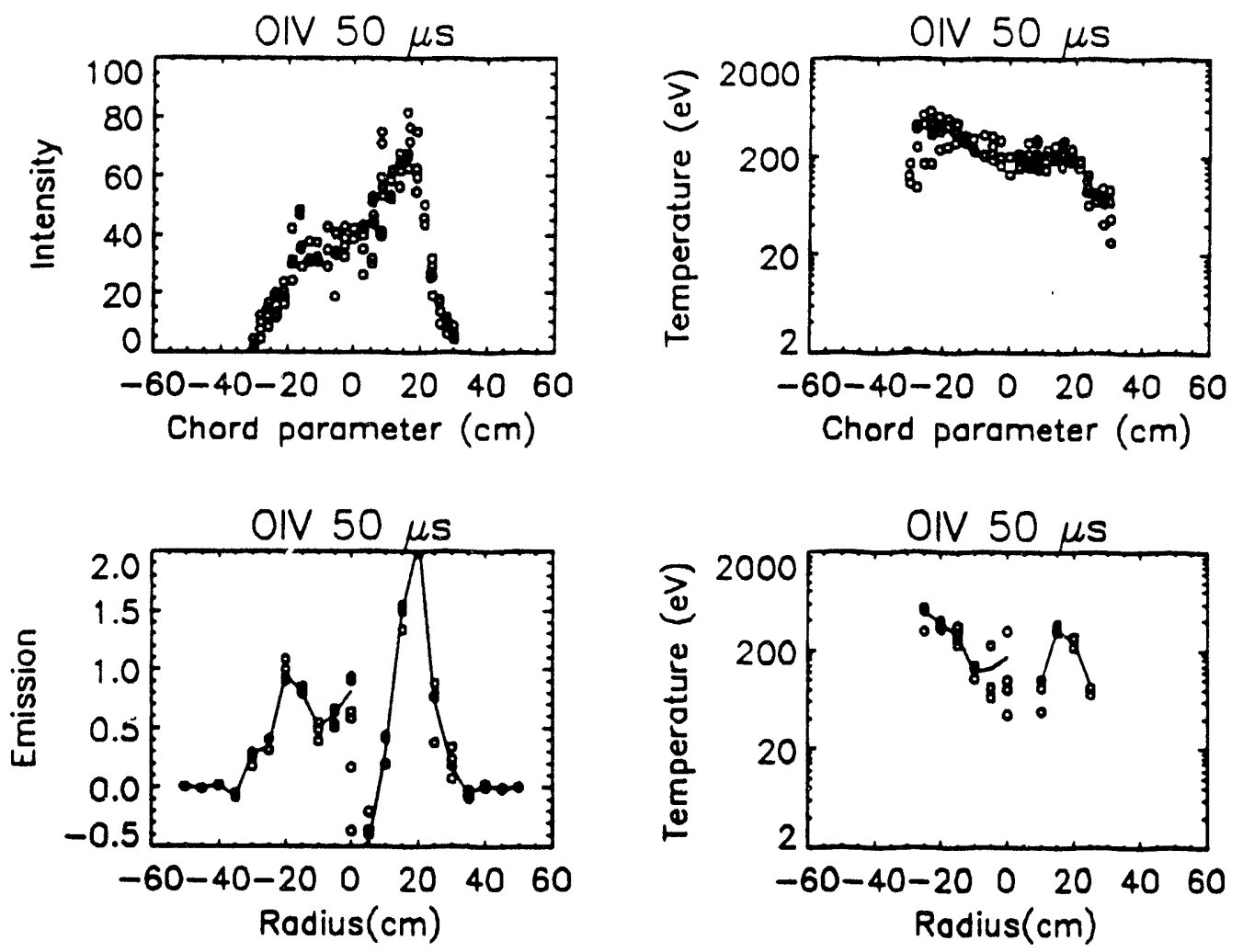

Figure 5.12: Top: Intensity and temperature line of sight measurements for OIV ( $3063 \AA$ ) at $50 \mu \mathrm{s}$. Bottom: The solid line is the inversion of these measurements. The circles are the inversion when noise is added to the data.

As a rule of thumb for oxygen lines, the square of the width measured in channels, is approximately equal to the temperature in $\mathrm{eV}$ when the spectrometer is set at $9000 \AA$. Typical lines will be 10 to 25 channels in width. The instrumental width is subtracted directly as a temperature. For a 5.5 channel instrumental width, about $30 \mathrm{eV}$ is subtracted from the inverted temperature:

$$
T_{\text {obs }}=T_{\text {line }}+T_{\text {instr }}
$$



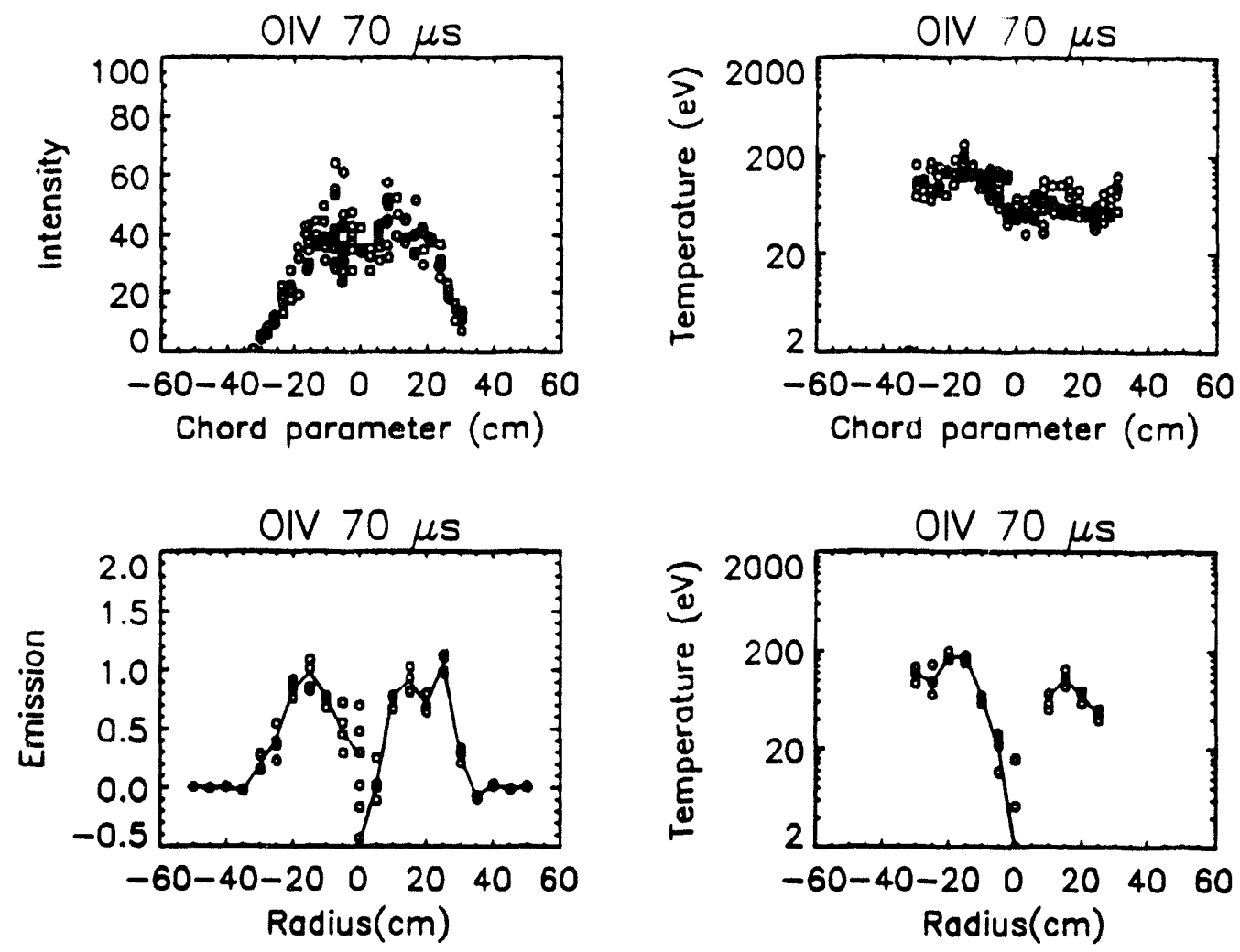

Figure 5.13: Top: Intensity and temperature line of sight measurements for OIV $(3063 \AA)$ at $70 \mu \mathrm{s}$. Bottom: The solid line is the inversion of these measurements. The circles are the inversion when noise is added to the data.

The ion temperature is markedly higher than the one obtained with the rotating slit, probably because of the addition of glass plates after the rotating slit measurements were made.

In principle, this inversion assumes toroidal symmetry. Although the data were not totally symmetric in the upper and lower midplane, assuming axisymmetry independently with top and bottom, the inversion shows the same general behavior in both halves. A fit was also performed using a polynomial fitting the data in both halves of the mid-planes all at once. Effectively, this 

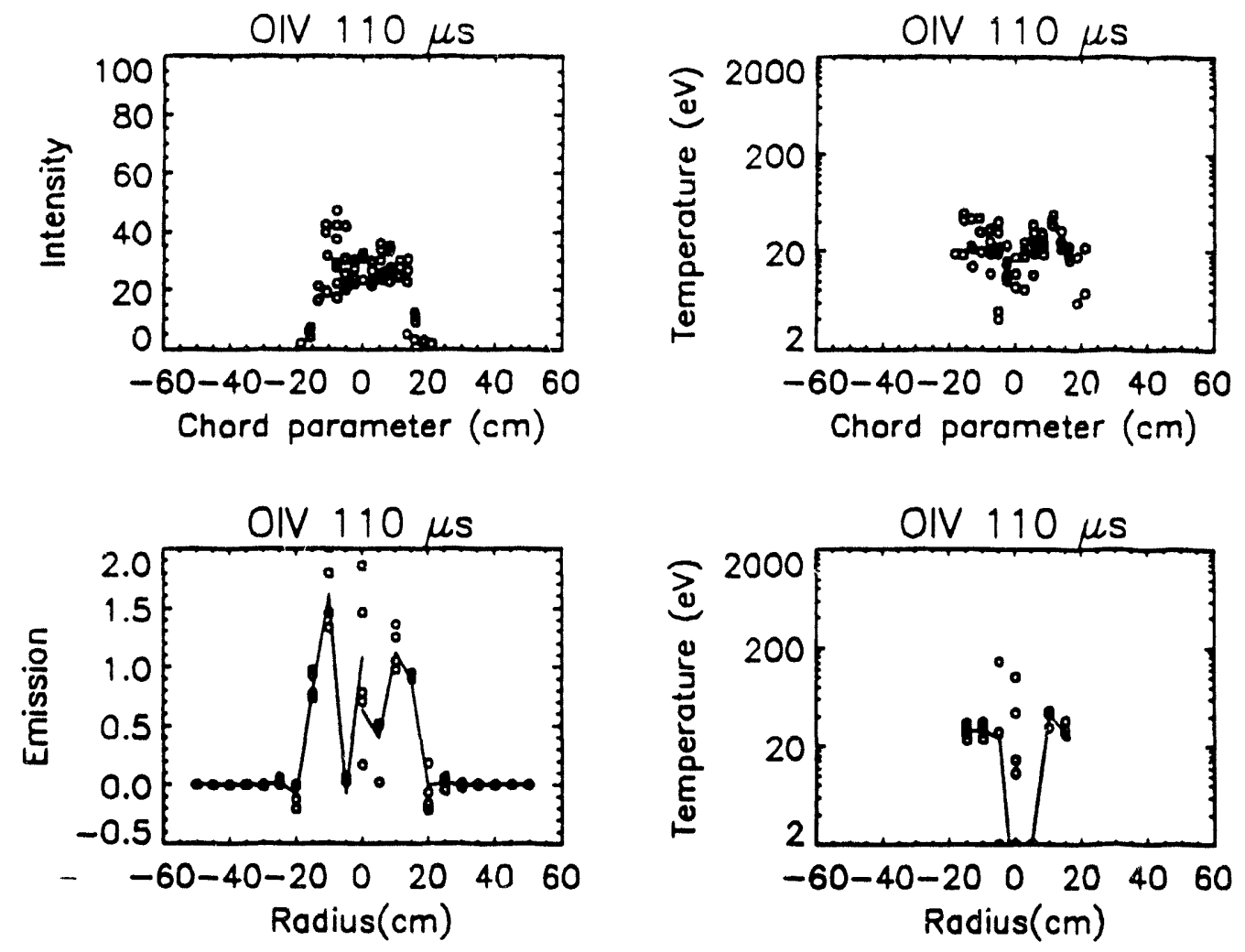

Figure 5.14: Top: Intensity ard temperature line of sight measurements for OIV $(3063 \AA)$ at $110 \mu \mathrm{s}$. Bottom: The solid line is the inversion of these measurements. The circles are the inversion when noise is added to the data.

symmetrizes the data or makes an average of the two sides. The inversion is of course symmetric and showed the same general behavior as the former inversion.

The non-uniformity in transmission of the lens system should cause small errors in the measured intensities. This error is minimized with the following procedures:

- The mirror was rotated such that there is an overlap in data between 

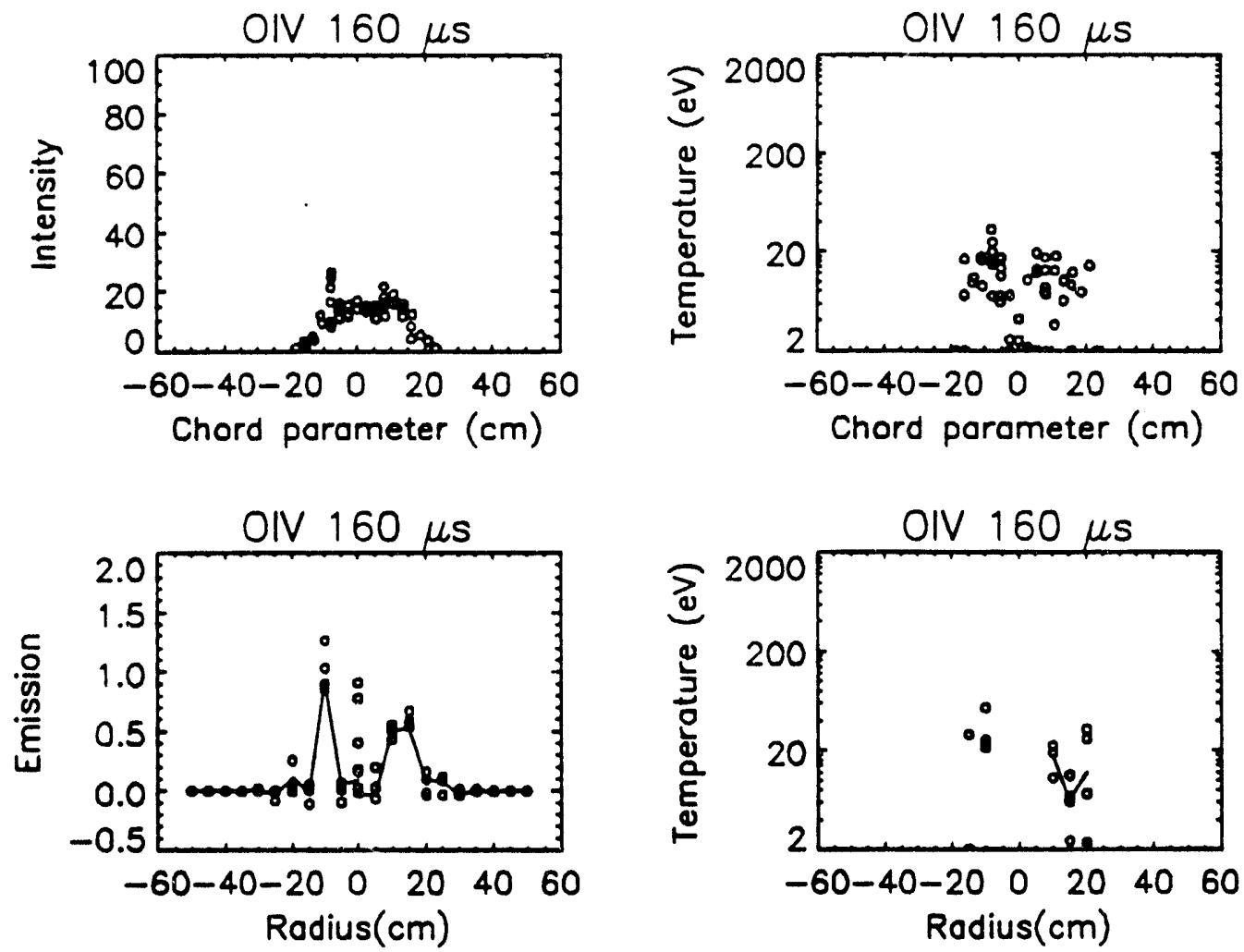

Figure 5.15: Top: Intensity and temperature line of sight measurements for OrV $(3063 \AA)$ at $160 \mu s$. Bottom: The solid line is the inversion of these measurements. The circles are the inversion when noise is added to the data.

subsequent angles, thereby averaging over different areas of the lens system.

- When a polynomial fit is applied to the raw data, those variations will be averaged out. 


\subsubsection{Plasma symmetry}

When performing the inversion, we assumed a toroidally invariant plasma, i.e, all quantities are independent of $\phi$, the toroidal angle. However, already from the raw data, we could observe an up-down asymmetry in intensity and in temperature. In the next chapter we will see that the magnetic scans showed $\mathrm{n}=1,2,3$ and higher modes of asymmetry.

These observations of non-axisymmetry indicate that the fine details of the inversion cannot be trusted. But the gross behavior (in this case emission forming a ring), is probably still correct. It would also be important to have invariance along the $z$-direction in order to extrapolate these results to offmidplane slices of the plasma. Not much can be inferred from spectroscopy since no scans were taken off midplane. Magnetic data show a left-right symmetry and therefore $z$-invariance around the midplane as shown in Fig. 3.3 Using a pin-hole camera, with a filter, we could observe regions of intense emissions around the 16 electrodes on each side of the machine as shown in Fig. 5.16. By setting the spectrometer in zeroth order and using the OMA as a time gated camera, as described in section 4.4.1, we could get time resolved pictures of the plasma. Different filters showed basically the same behavior as shown in Fig. 5.17 and 5.18. Looking from the end, we can see intense regions of emission around each electrode, indicating an $n=16$ patern for $z= \pm 20 \mathrm{~cm}$. However no information is given for the midplane with those measurements. These two- dimensional data show a toroidal asymmetry and a strong dependence on $\mathrm{z}$ for the light emission in the vicinity of the electrodes. An important fraction of the radiation seems to originate from around the 


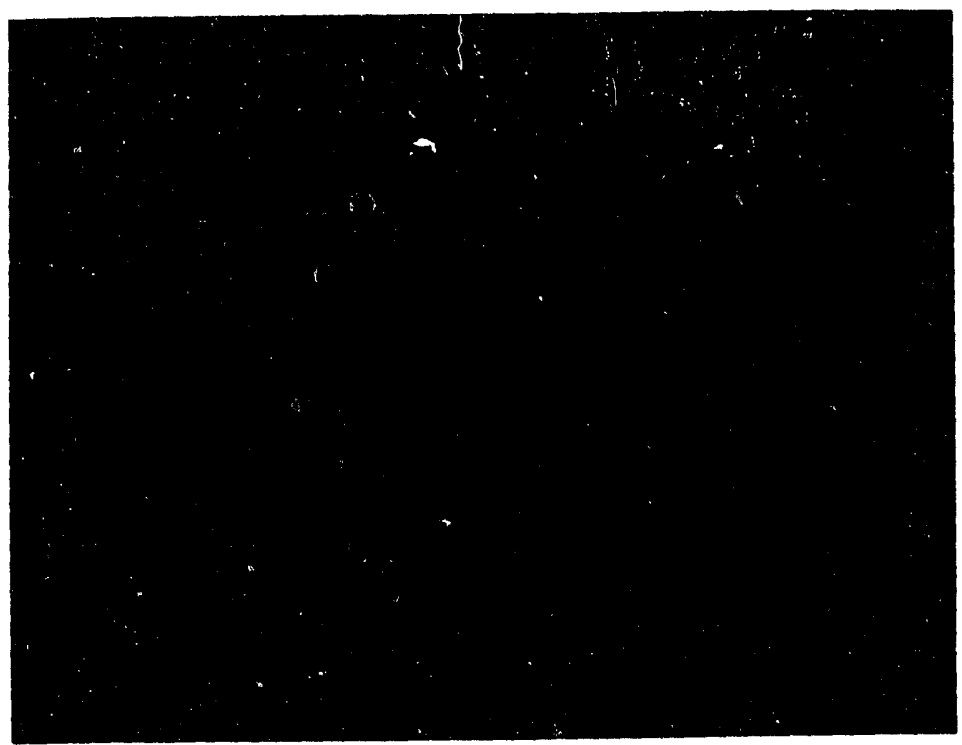

Figure 5.16: Picture of the plasma discharge taken with a pin-hole camera. End view.

electrodes. Possibly a plasma sheath is present.

In summary, from the magnetic field measurements we can assume that the inverted radial behavior of OIV light emission is possibly valid for regions near mid-plane, away from the electrodes, for $|z|<1 \mathrm{~cm}$. A toroidal asymmetry exists. From light emission data, we see an $n=1$ assymetry in the midplane and $n=16$ at the electrodes; from magnetic data, $n=1,2,3$ modes are present in the midplane. 

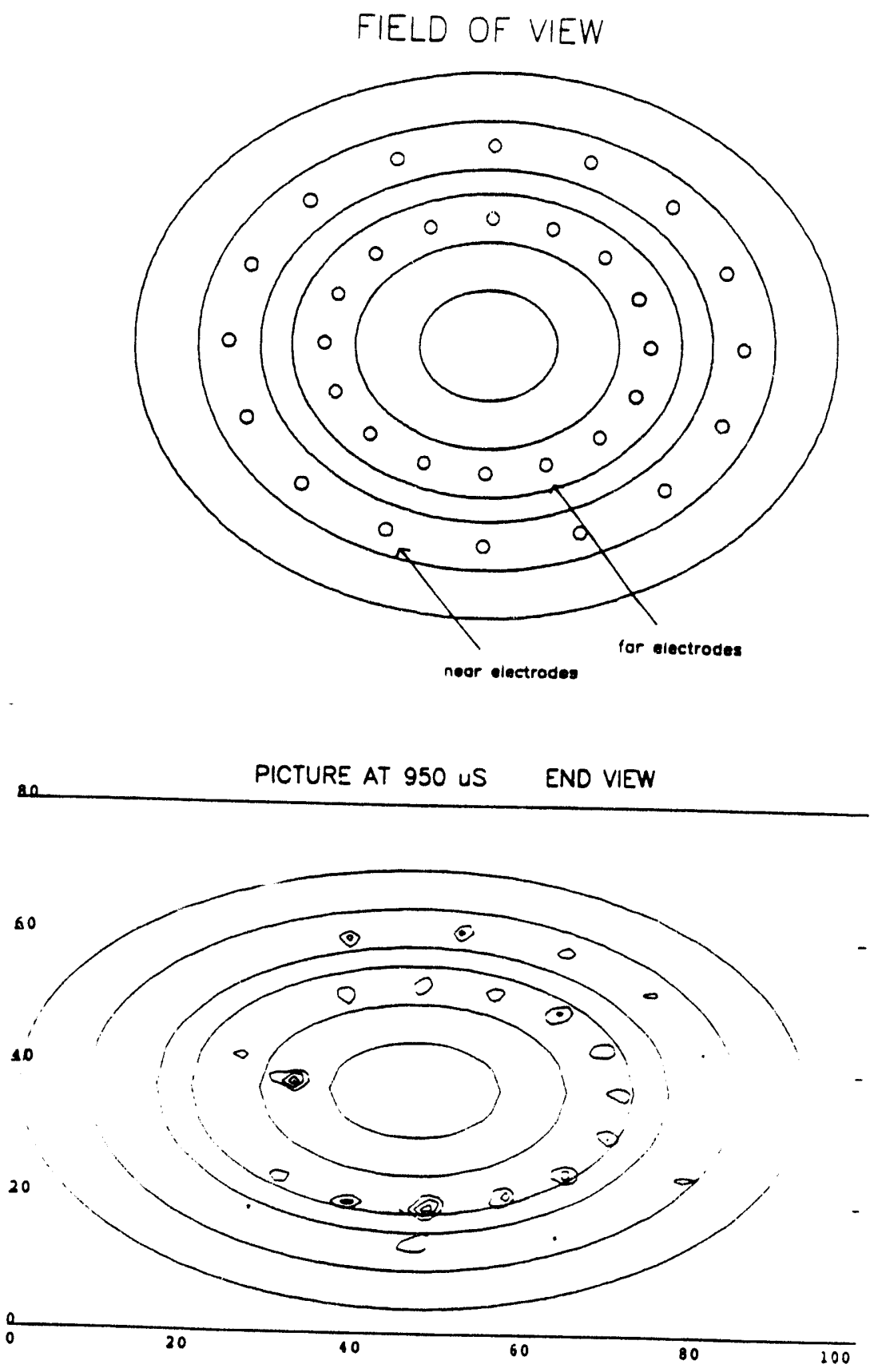

Figure 5.17: Top:Reference field of view diagram showing the position of the electrodes. Bottom: Contour of constant intensity of the plasma discharge taken with the OMA at $950 \mu s$. End view. 

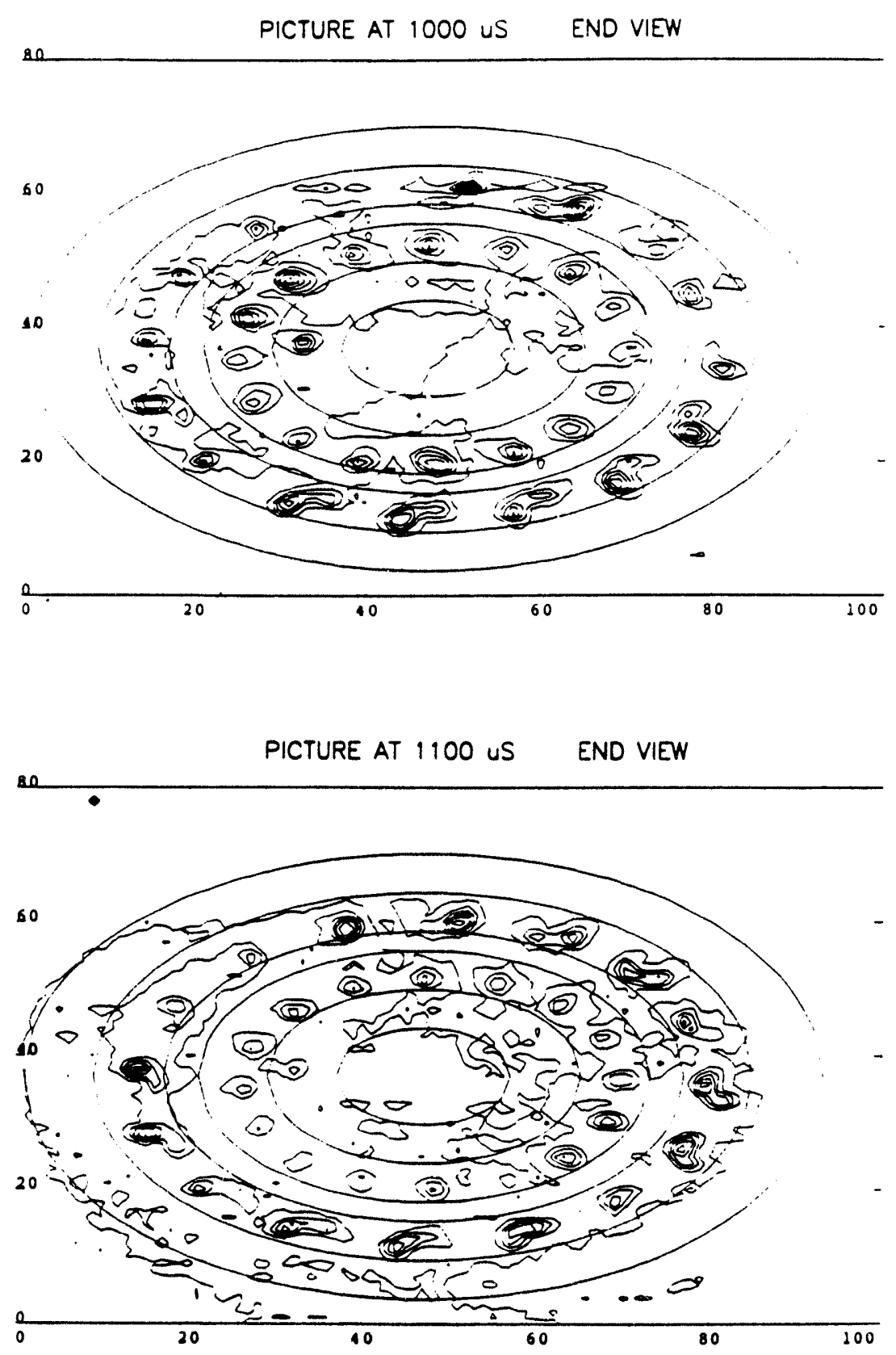

Figure 5.18: Contour of constant intensity of the plasma discharge taken with the OMA at 1000 and $1100 \mu s$. End view. 


\section{Chapter 6}

\section{Discussion and Conclusion}

The inversion of OIV gave peaks in emission at very specific radii as a function of time. Figure 6.1 shows the $z$ component of current density as a function of radius for different times in the midplane. Comparing with Figs. 5.11 to 5.15 we see that the radii of maximum emission correspond to the radii of maximum current density. From [37], OIV has a maximum fractional abundance at an electron temperature of $15 \mathrm{eV}$. For a temperature above $15 \mathrm{eV}$, the radial profile well resolved would have been hollow at the radius of maximum electron temperature. This puts an upper bound of $15-20 \mathrm{eV}$ on the peak electron temperature. On the other hand, the observation of OVI with the VUV spectrometer puts a lower bound of also $15-20 \mathrm{eV}$.

In this chapter we will consider classical heating, instability wave oscillations, helicity transport, tearing modes and X-point heating as possible mech- 

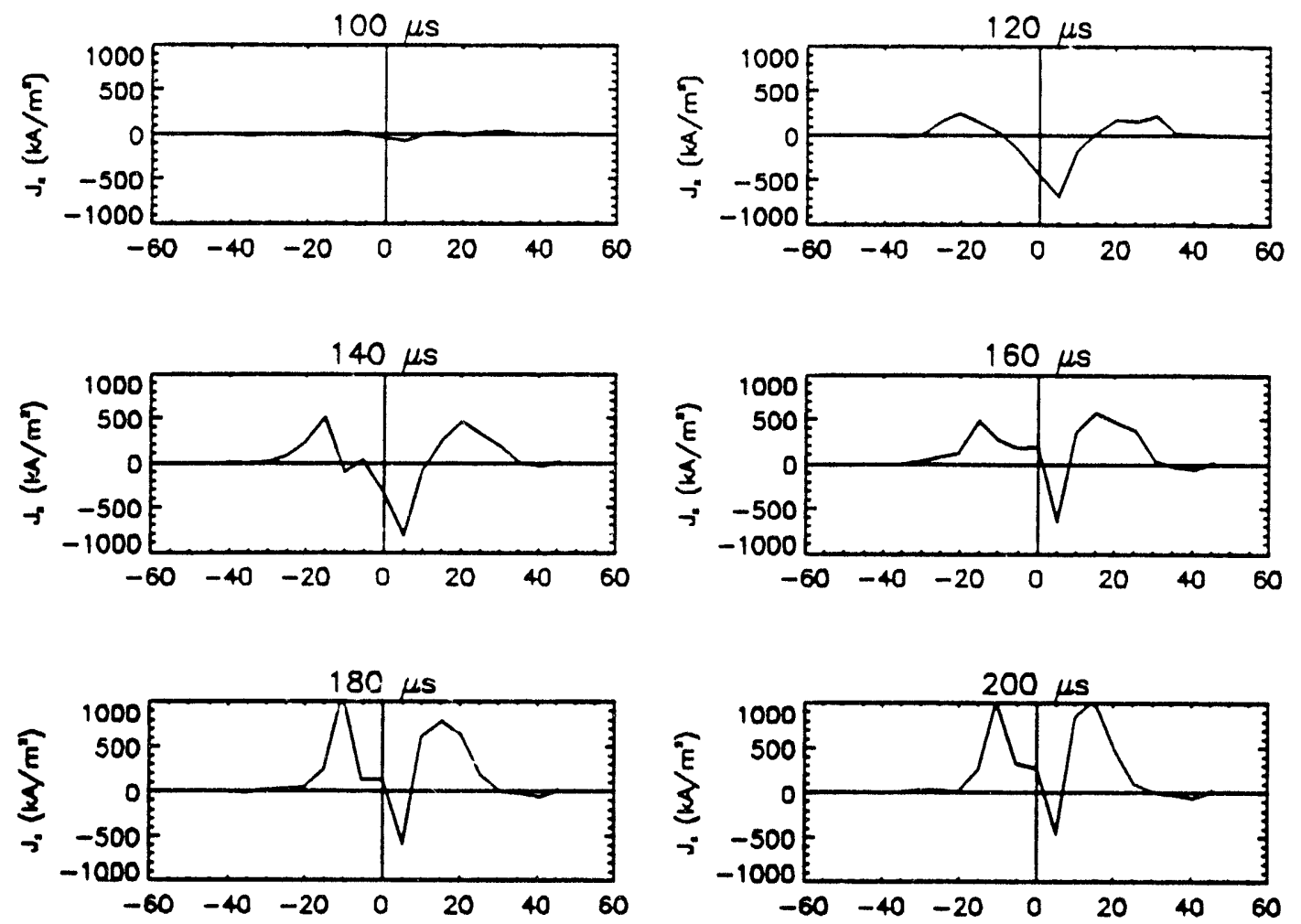

Figure 6.1: Current density as a function of radius in the midplane. With glass plate barriers.

anisms for heating the ions. We will see that the most likely mechanism to occur in MS is the $\mathrm{X}$-point heating.

Classically with Ohmic heating, a local electric field is applied to the particles and the electric force is balanced by collisions: $e \mathbf{E}=\mathbf{F}_{\mathbf{c}}$ in steady state for each species. For a plasma made of electrons and ions, conservation of momentum gives

$$
m_{e} \overline{\mathrm{V}}_{e}=-m_{\mathrm{i}} \overline{\mathrm{V}}_{\boldsymbol{i}}
$$


The ratio of power given by the electric field to each species is

$$
\frac{P_{e}}{P_{i}}=\frac{e E \overline{V_{e}}}{e E \overline{V_{i}}}=\frac{m_{i}}{m_{e}}
$$

actually The electrons gain energy at a rate $m_{\mathfrak{i}} / m_{e}$ faster than the ions due to the electric field. Classically, the ions gain most of their energy through collisions with the electrons. Therefore, the electrons should have the highest temperature. The temperatures measured for OIV are two orders of magnitude higher than the electron temperature at early times and this cannot be explained classically.

A candidate for ion heating is the ion acoustic oscillations [10]. However this mode is stabilized by Landau damping unless $T_{i} \ll T_{e}$. Other candidates are the ion cyclotron oscillations and magnetosonic oscillations[48]. We need respectively,

$$
V_{d}>15 \frac{T_{i}}{T_{e}} V_{i}
$$

and

$$
V_{d}>V_{A}
$$

where $V_{d}$ is the drift velocity and

$$
V_{i}=\left(\frac{2 T_{i}}{m_{i}}\right)^{1 / 2}
$$

In MS, $V_{A}=10 \mathrm{~cm} / \mu \mathrm{s}$, the maximum $V_{d}$ observed is about $6 \mathrm{~cm} / \mu \mathrm{s}$. For deuterium at $100 \mathrm{ev}, V_{i}=10 \mathrm{~cm} / \mu \mathrm{s}$ and $T_{i} / T_{e}=10$. Those two modes should not get excited. 
Anomalous ion heating has been observed in S-1 [6] and CTX [7] spheromaks. It has also been observed in RFP experiments: ZETA [38], ZT-40M [39], HBTX [40] and REPUTE-1 [10]. Because an RFP [26] operates in steady state, the data are easier to analyze. Looking at RFP data, Jarboe and Alper [41] concluded that the highly resistive edges of the RFP leads to high helicity loss. Because the plasma tends to stay in the Taylor state [27], the MHD activity increases in order to try to maintain $\mu$ constant. This increased activity might lead to power loss to the ions, although the exact mechanism is not understood. Some computations on CTX showed that $35 \%$ of the poloidal flux penetrated the mesh boundary of the flux conserver and therefore encountered cold regions of plasma [42]. Due to that field error, the edges become very resistive and as for the RFP could create high ion heating. After replacing the flux conserver with a new one that minimizes the field error [43], they obtained remarkable improvements in their plasma lifetime and electron temperature. But first of all the ion temperature dropped by a factor of two, an indication that they were successful in controlling the turbulent heating due to field error. 


\subsection{Helicity transport and anomalous ion heating}

\subsubsection{Results in RPF's}

Like a tokamak, an RFP consists of a toroidal plasma with a transformer coil through its center, but like the spheromak it relaxes to a force-free state after formation [44]. An external toroidal current is ramped up and maintained at a constant value. The plasma acts like the secondary of a transformer and a loop voltage creates and for the most part maintains the plasma toroidal current. A dynamo can also develop, generating some of the current. Under certain conditions, in the relaxed Taylor state, there is a reversal of magnetic field and current at the outer edge of the RFP [44]. The outer electric field and the main electric field have opposite directions. This is not possible according to classical Ohm's law assuming Spitzer resistivity only. By some process, the plasma must set up non-classical electric fields in order to relax toward the Taylor state.

The following observations were made in RFP's in their steady-state: there is correlation between the onset of magnetic field fluctuations, anomalous high ion temperatures, and non flat $\mu$ profiles, where

$$
\mu=\frac{\mathbf{J} \cdot \mathbf{B}}{B^{2}} .
$$

It is suspected that those effects have a common cause and are related to 
transport of magnetic helicity. As a test, a tile was inserted in the outer edge in order to intercept a magnetic flux $\Psi$ [45]. If a plasma sheath appears at the tile surface, then there is a drop of potential $\chi$ and helicity is extracted at a rate $\Psi \chi$. This would then enhance helicity transport. It is then ohserved that fluctuations and ion temperature are enhanced as a result. Some theories look at fluctuations as a mean of transporting energy and magnetic helicity from one region of the plasma to the other $[46,47]$. If the fluctuations give part of their energy to the ions, then the transport process becomes less efficient.

\subsubsection{Energy and helicity global balance}

Taking into account fluctuations, in steady state, the global balance equations for helicity and energy for a given closed flux tube are [46],

$$
\begin{aligned}
V \Psi_{t} & =\int_{v} \eta \mathbf{J} \cdot \mathbf{B} d^{3} x+\int_{v}<\tilde{\mathbf{U}} \times \tilde{\mathbf{B}}>\cdot \mathbf{B}_{0} d^{3} x \\
V I_{t} & =\int_{v} \eta J^{2} d^{3} x+\int_{v}<\tilde{\mathbf{U}} \times \tilde{\mathbf{B}}>\cdot \mathbf{J}_{0} d^{3} x .
\end{aligned}
$$

Here $V$ is the loop voltage, $\Psi_{t}$ the toroidal magnetic flux, $I$ the total toroidal current, $\tilde{\mathbf{U}}$ and $\tilde{\mathbf{B}}$ are the fluctuating parts of the velocity and magnetic fields, while $\mathbf{J}_{\mathbf{0}}$ and $\mathbf{B}_{\mathbf{0}}$ are unperturbed quantities. In steady-state, the helicity injected in a given volume is balanced by dissipation and a term involving transport through fluctuations. It can be shown [46] that if there is no helicity crossing the boundary of the volume, then

$$
\int_{v}<\tilde{\mathrm{U}} \times \tilde{\mathbf{B}}>\cdot \mathbf{B}_{0} d^{3} x=0
$$


which means that there are no losses of helicity associated with the transport process. On the other hand. the power injected is balanced by classical resistive losses through Spitzer resistivity and a term involving transport through fluctuations. If there is no energy crossing the boundary of the volume, then

$$
\int_{v}<\tilde{\mathbf{U}} \times \tilde{\mathbf{B}}>\cdot \mathbf{J}_{0} d^{3} x=[\text { loss }] \geq 0
$$

This integral does not necessarily vanish [46] and could represent power loss to ions.

We can see that $<\tilde{\mathbf{U}} \times \tilde{\mathrm{B}}>$ has the dimension of electric field. Fluctuations are extracting energy and helicity by applying a local electric field opposite in direction to the local $\mathbf{J}_{\mathbf{0}}$. In the ideal case where there is no losses the fluctuations will produce an electric field elsewhere in the same direction as the local $\mathbf{J}_{\mathbf{0}}$ and give jack the extracted energy and helicity. This could be the origin of the non-classical electric field in RPF's [46,44]. The fluctuations will create and maintain the Taylor state. When non resistive losses occur, all the energy is not transferred, the necessary current to stay in the Taylor state is not maintained and the $\mu$ profile is not flat anymore. When a tile is inserted in the plasma, the $\mu$ profile drops at the edge and helicity is transported from the inner region to the outer region, to replace the extracted helicity. The transport is enhanced and so is the ion heating and the amplitude of fluctuations. 


\subsubsection{Modified Ohm's law}

In an effort to explicitly calculate the power given to the ions by helicity transport, Boozer derived a modified Ohm's law [47] for a force-free plasma in the presence of fluctuations.

$$
\mathbf{E}+\mathbf{V} \times \mathbf{B}=\eta \mathbf{J}-\mathbf{R}_{\mathbf{f}}
$$

where $\mathbf{R}_{\mathbf{f}}$ is the electric field created by the fluctuations. He shows that the contribution of this field to energy dissipation is given by,

$$
\frac{P_{\text {ion }}}{V o l}=\lambda(\nabla \mu)^{2}
$$

where $\lambda$ has the form of a viscosity preventing $\mu$ from changing over a spatial scale shorter than

$$
\delta^{2}=\frac{\lambda}{\eta B^{2}}
$$

The derivation is shown in the appendix.

The formula developed by Boozer was used to try to calculate explicitly the expected power going to the ions from the measured $\mu$ profile. A certain inaccuracy is expected because at formation the plasma is not force-free.

According to Fiameiri and Bhattacharjee [49], $\delta$ has the following order of magnitude, assuming a cylindrical plasma,

$$
\delta=O\left[\frac{a \gamma^{\prime}}{\Delta^{\prime}} \beta\left|\frac{\tilde{B}}{B_{0}}\right|\right]
$$


where $a$ is the radius of the plasma. $\gamma^{\prime}=d \gamma / d r, \gamma=r B_{z} / B_{\phi}$ in MS coordinates, $\beta$ is the plasma beta, $\Delta^{\prime}$ is related to the growth rate of tearing modes and is typically equal to 10 in RFP's and $\tilde{B} / B_{0}$ is the relative magnitude of the fluctuating field. $\delta$ is typically equal to $1 \mathrm{~cm}$ in RFP's.

In the early formation phase of MS, the magnetic configuration consists of a z-discharge in an almost constant $z$ magnetic field. Because of the reversed current, $B_{\phi}$ changes sign and $\gamma^{\prime}$ and $\delta$ go to infinity wherever $B_{\phi}$ vanishes. Figure 6.3 shows the radial behavior of $q$, which is proportional to $1 / \gamma$, at different times for conditions without glass plate barriers. $\delta$ was derived by assuming that a bath of low intensity tearing modes (fluctuations) is present and therefore probably breaks down when $\delta$ goes to infinity. Therefore, it is not possible to calculate explicitely the power going to the ions from helicity transport.

\subsection{Tearing modes}

Even though the spectroscopic data had an up-down asymmetry, we assumed a toroidal symmetry in our analysis up to now. We decided to investigate more carefully ${ }^{1}$ the axisymmetry of our plasma from magnetic probe data. Figure 6.2 shows contours of constant value of toroidal flux in the midplane for conditions without glass plate barriers, where reverse current was also observed. We can see clearly toroidal $n=2$ modes forming as indicated by the formation of magnetic islands and "current" islands at $160 \mu \mathrm{s}$. The two

\footnotetext{
${ }^{1}$ Following a suggestion from Dr. John Finn.
} 
islands merge to form an $n=1$ mode at about $200 \mu \mathrm{s}$. The modes dissipate as the reversed current disappears. An $n=3$ mode might be present at earlier times.
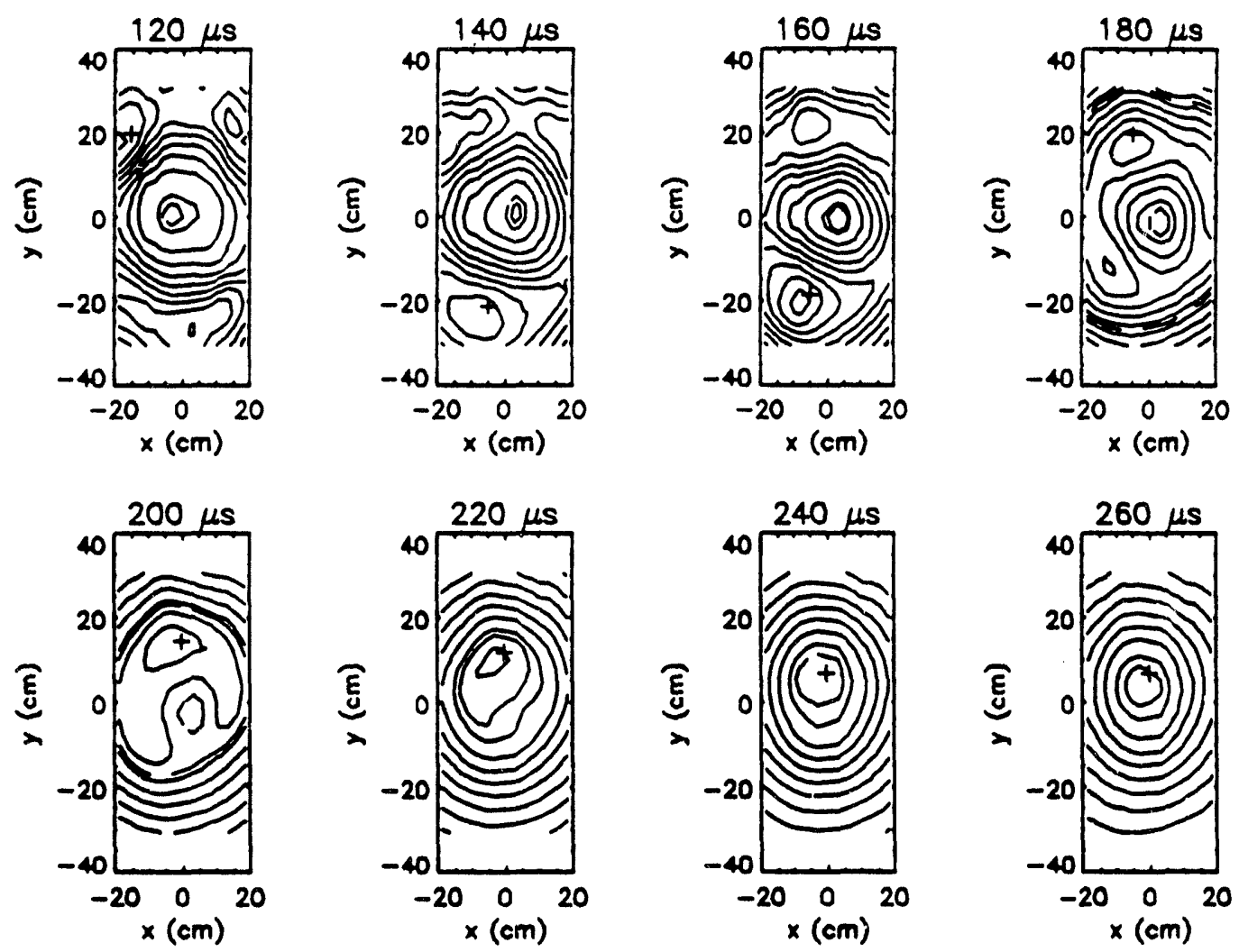

Figure 6.2: Contours of constant $r-\phi$ flux in the midplane for conditions without glass plate barriers, obtained by integrating the toroidal and radial fields. The flux is chosen to be zero at the origin. The dotted lines are contours of zero flux. The symbol ' $t$ ' identifies the minimum of flux. We can see an initial $n=3$ mode that becomes $n=2$ and finally $n=1$.

A tearing mode instability çreates flamentation of the main (toroidal) current and creates islands of magnetic field in tokamaks. In the process, the poloidal magnetic energy is reduced. A parameter characterizing the appearance of tearing modes is the safety factor $q$. In a small aspect ratio torus, $q$ is 
difficult to calculate from experimental data. In MS. we will approximate the safety factor using the large aspect ratio form of $\mathrm{q}$,

$$
q(r)=\frac{2 L B_{\phi}}{2 \pi r B_{z}},
$$

where $r$ is the radial position and $L$ is the length of the plasma. It is a measure of the number of toroidal turns per poloidal turn that one obtains when following a magnetic field line until it reconnects.

Tearing mode instabilities occur on rational surfaces for $q=m / n$ where $m$ is the poloidal mode and $n$ is the toroidal mode of instability, in MS geometry. : 3 ure shows the behavior of $q$ as a function of radius in the midplane for different iiñies.

The radii where the islands occur appear to be just the same radii where q goes to zero, for each time frame. This suggests an $m=0$ mode, a mode that is independent of $z$. Furthermore, the islands disappear when the reverse current vanishes and q stays always positive. The observed $q$ profile would not be consistent with a double tearing mode instability. As long as the reverse current is maintained, the $n=2$ mode seems to persist. We then have opposite currents adjacent to each other and they repulse each other keeping the system in equilibrium until the reverse current vanishes. Then the islands merge into one island.

The classical diffusion time of the magnetic field is estimated to be

$$
\tau_{d}=\frac{B \pi}{c \frac{d}{d x}\left(\eta \frac{d}{d x}(B)\right)} \sim 100 \mu s
$$



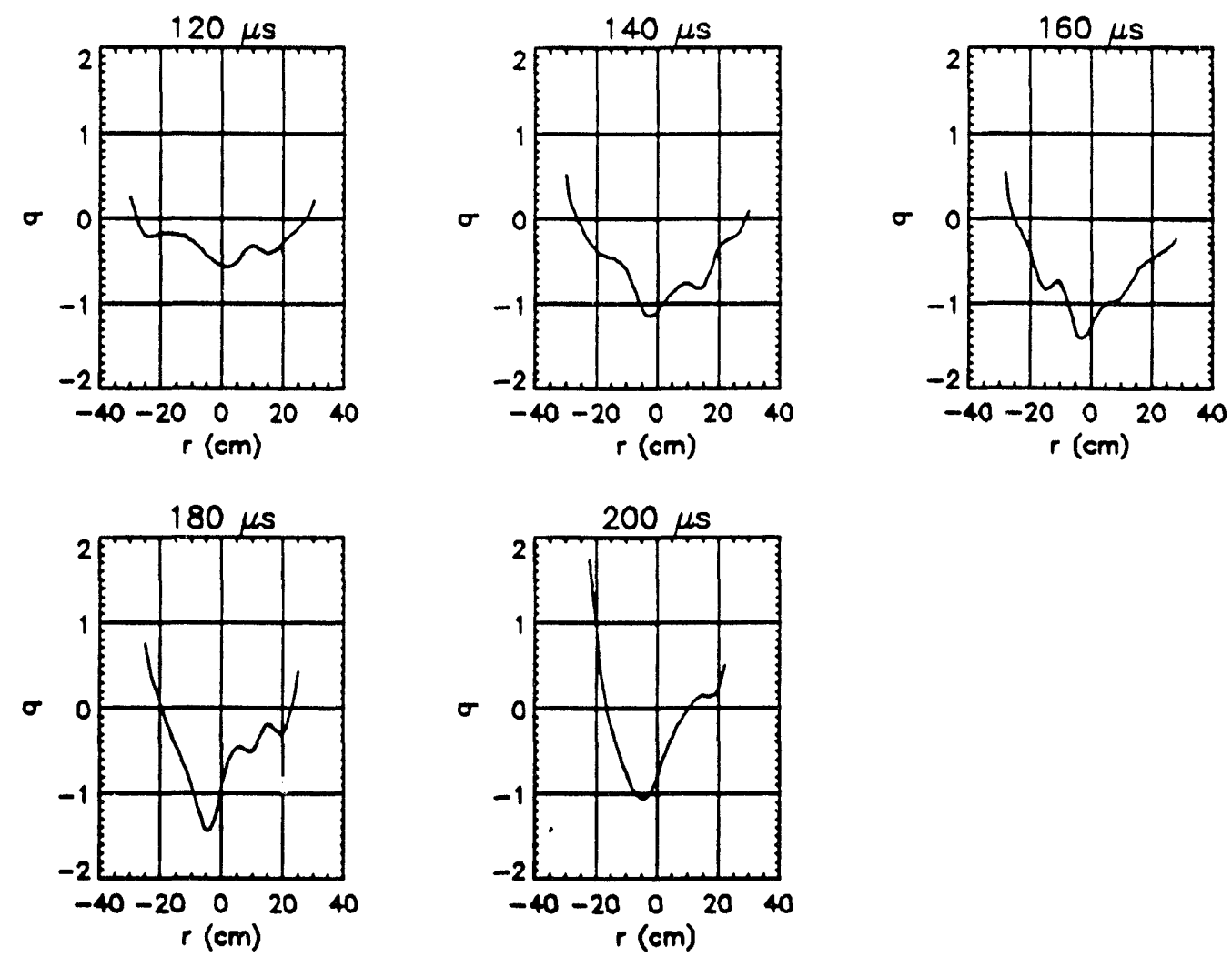

Figure 6.3: Radial profile of the safety factor $q$ in the midplane for conditions without glass plate barriers.

using $Z_{\text {eff }}=1$. This is about the time scale observed for the current density to become uniform at $r=0$. Therefore the tearing mode instability is not growing fast enough to change the diffusion appreciably from the classical diffusion time.

Another possibility, is that the islands are associated with current filaments connecting individual electrodes. The 16 flux surface islands would be difficult to observe because we average over many shots. What would be the magnetic 
energy released per particle if initially we start with 16 filaments of current and then form an $n=8$ mode? Let us consider two adjacent filaments. of current $I$, of radius $r_{0}$ and separated by a distance $d$. The force on an electron of the first filament due to the field of the second filament is given by,

$$
F=B \frac{I}{n}
$$

where $\mathrm{n}$ is the electron density in the filaments. As the filaments merge, the energy given per electron is,

$$
W=\frac{\mu_{0} I^{2}}{4 \pi n}\left(\ln \frac{d}{r_{0}}+\frac{1}{2}\right)
$$

At $t=20 \mu \mathrm{s}$ in MS, we have in the order of $10^{4} \mathrm{~A}$ per electrode. Assuming the filaments radii to be the same as the electrode's then $r_{0}=1 \mathrm{~cm}$ and $d$ should be about $10 \mathrm{~cm}$. We then obtain $2 \mathrm{eV}$ energy per electron. If the filament moves as a whole, this energy is actually coupled to the ions. The main species would get $2 \mathrm{eV}$ and oxygen would get $16 \mathrm{eV}$. This is an upper bound since due to the presence of the other filaments, the actual magnetic field is reduced. This is more than order of magnitude smaller than the observed temperature.

The islands appear to move at a slow pace. We can see displacements of up to $10 \mathrm{~cm}$ per $10 \mu \mathrm{s}$ time frame. If this gets transformed into thermal energy, then the gains are $1 \mathrm{eV}$ per deuteron and $8 \mathrm{eV}$ pes oxygen ion.

In conclusion, exploring the possibility of ion heating through helicity transport, we could not evaluate explicitly its contribution because of the vanishing 
toroidal current at a given radius. When looking for toroidal symmetry, we could observe $n=3$ magnetic islands evolving into an $n=1$ island, indicating possibly tearing modes instability. The structure is consistent with an $m=0$ mode. Even though we observe tearing modes simultaneously to ion heating, a simple estimate shows that this mechanism will not provide sufficient energy to the ions to match our experimental ion temperature observations.

\subsection{X-point heating}

The non-uniformity of the $I_{z}$ current causes X-points to form in the magnetic surfaces. An X-point corresponds to a saddle point in the graph of the toroidal magnetic flux versus $x$ and $y$ where $x$ and $y$ are the mid-plane coordinates. It has been shown $[50,51]$ that $\mathrm{X}$-points have an important role in ion heating.

Some definitions:

X-point: In figure 6.4 are shown simulation contours of constant magnetic flux for four parallel current sources (simulating the electrodes) flowing in the same direction, for two different cases. For the case of no reversed current, at $x=0, y=0$ the resultant magnetic field is zero and we can see that the magnetic surfaces cross each other, creating a multiple $\mathrm{X}$-point. In the second case, a reverse current equal to half of the total forward current is added with uniform density for a radius smaller than the electrode radius. We can see similarities between the $\mathrm{X}$-point forming in this case and the experimental magnetic data. 

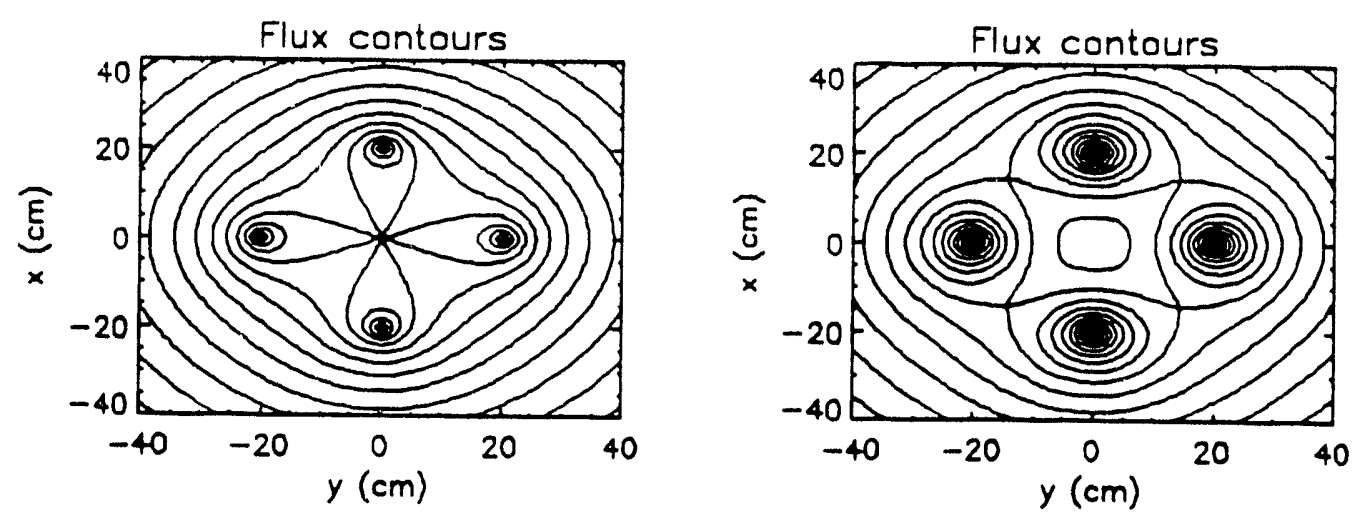

Figure 6.4: Simulation contour of constant flux for an $n=4$ island mode. Left: no reverse current. Right: reverse current equal to $50 \%$ of the total forward current.

$\mathrm{X}$-point heating: If an electric field exists in the vicinity of the $\mathrm{X}$-point then an $\mathbf{E} \times \mathbf{B}$ drift could take place. For MS, the drift is in the plane at right angle to the machine symmetry axis. For an electric field in the same direction as the current, then a radial pinching effect takes place. In Fig. 6.4, a flow of particles would converge toward the $\mathrm{X}$-point in the $r$-direction and a flow would diverge along the $\phi$-direction. For a reversed electric field, the flows are reversed. Heating takes place when the energy associated with the flow is converted into thermal energy due to viscosity.

In their 2-D simulation of MS formation [50], Guzdar and Finn, found strong ion heating to occur near the $\mathrm{X}$-point of the reversal coils. Ion heating rates on the order of $100 \mathrm{eV} / \mu \mathrm{s}$, raising the ion temperature to over 400 $\mathrm{eV}$ are predicted at formation. However, observations in MS showed no ion heating to occur near the reversal X-point which is at around $r=30$ to $35 \mathrm{~cm}$. 
All the heating is observed for $r<25 \mathrm{~cm}$, approximately at the radii of the observed magnetic islands. As many as 3 islands can be seen at $t=20 \mu \mathrm{s}$. The filamentation of the current could cause viscous ion heating associated with the X-points. Guzdar and Finn's simulation could not take into account non-axisymmetric effects and hence X-point heating associated with $I_{z}$ filamentation was not predicted.

\subsubsection{Heating estimates}

Using a one fluid model [52], we attempt to simulate the temperature behavior using the following simplified set of equations.

$$
\begin{gathered}
\rho d \mathbf{V}_{\perp} / d t=J_{z} \hat{\mathbf{z}} \times \mathbf{B}_{\perp}+\eta_{0} d^{2} V_{\perp} / d X^{2}-\nabla p \\
n d T_{i} / d t=\eta_{0}(d V / d X)^{2}-n\left(T_{i}-T_{e}\right) / \tau
\end{gathered}
$$

where $\tau$ is the characteristic time for ion temperature to decay due to thermal equilibration with electrons and $\eta_{0}$ is a viscous coefficient [52] defined as,

$$
\eta_{0}=3 \times 10^{12} \mathrm{Ti}^{5 / 2}\left(\mathrm{eV} \mathrm{sec} \mathrm{m}^{-3}\right)
$$

Transport and charge exchange were neglected. $\mathbf{V}=\mathrm{V}_{\perp}$ is perpendicular to $\hat{\mathbf{z}}$. We neglect the term $\nabla p$ in the force equation 6.6 because we are interested in the plasma at early times. We take $d V / d X$ to be $V / L$ and $d^{2} V / d X^{2}$ to be $V / L^{2}$ where $L$ is a typical scale length. We assumed $V$ to be zero at the $\mathrm{X}$-point. 
From the experimental data, we approximate the different parameters as

$$
\begin{aligned}
J_{z} & \sim \frac{10^{4}}{\omega} \sin \omega t\left(\mathrm{~A} / \mathrm{m}^{2}\right) \\
B_{\perp} & \sim \frac{10^{-3}}{\omega} \sin \omega t(\mathrm{~T}) \\
\mathrm{J} \times \mathrm{B} & \sim \frac{10}{\omega^{2}} \sin ^{2} \omega t\left(\mathrm{~N} / \mathrm{m}^{2}\right) \\
n & \sim 10^{20}\left(\mathrm{~m}^{-3}\right)
\end{aligned}
$$

where $\omega=\pi / 150 \mu \mathrm{s}^{-1}$ and $t$ is in $\mu \mathrm{s}$.

At $t=0$ there is no flow motion and Ohm's law,

$$
E_{z}+(\mathrm{V} \times \mathrm{B})_{z}=\eta J_{z}
$$

becomes

$$
E_{z}=\eta J_{z}
$$

and equation 6.6 gives $\rho d \mathbf{V} / d t=\mathbf{J} \times \mathbf{B}$. We then estimate that after $1 \mu s, \mathbf{V} \times \mathbf{B}$ becomes larger than $\eta \mathbf{J}$. The plasma is then described by MHD equations and the frozen-in theorem holds. The power delivered to the plasma, E. J mainly goes into the ions through $(\mathbf{J} \times \mathbf{B}) \cdot \mathbf{V}$. At some later time the viscous friction term in equation 6.6 will balance or become larger than $\mathbf{V} \times \mathbf{B}$ term. This is when appreciable ion heating begins to take place. By scaling equations 6.6 and 6.7 , and using $\sin \omega t \cong \omega t$, we get:

$$
\begin{aligned}
\frac{\rho V}{t} & =J B=1 \times 10^{13} t^{2} \\
\frac{n T_{i}}{t} & =3 \times 10^{12}\left(\frac{V}{L}\right)^{2}
\end{aligned}
$$


where $t$ is in seconds and $L$ is a typical scale length and is taken to be $.1 \mathrm{~m}$ which is the approximate distance between adjacent electrodes.

From eq. 6.10, $V=10^{20} t^{3}$. From eq. 6.11 .

$$
\begin{aligned}
1 / t & =\frac{3 \times 10^{12}(V / L)^{2} T_{i}^{3 / 2}}{n} \\
& =\frac{3 \times 10^{12} \times 10^{40} T_{i}^{3 / 2} t^{6}}{.01 \times 10^{20}} \\
t^{7} & \sim 10^{-34} T_{i}^{3 / 2} \\
t & \sim 10^{-5} T_{i}^{3 / 14}(\mathrm{sec})
\end{aligned}
$$

For an initial ion temperature between 1 and $10 \mathrm{eV}$ we find $t \sim 10 \mu \mathrm{s}$. This is the typical time for the temperature to start to rise. Because of the nonlinearity of the equations, the rise could be sudden. The energy released is $100 \mathrm{eV}$ for the deuterons if after $10 \mu \mathrm{s}$ all the motion energy is transformed into temperature. Through collisions, oxygen is expected the gain the same velocity as deuterium and could heat up to $800 \mathrm{eV}$.

Integrating equations 6.6 and 6.7 numerically, gives the gross time behavior of $V$, the mass velocity, $T_{D}$ and $T_{O}$, the deuterium and oxygen ion temperatures, respectively. The thermal equilibration between electrons, deuterium and oxygen species was taken into account,

$$
\frac{d T_{\alpha}}{d t}=\sum \nu^{\alpha / \beta}\left(T_{\beta}-T_{\alpha}\right)
$$

Table 6.1 gives the thermal equilibration times for the species at differert temperatures. 


\begin{tabular}{|r||c|c|c|}
\hline$T_{\alpha}:$ & $10 \mathrm{eV}$ & $100 \mathrm{eV}$ & $1000 \mathrm{eV}$ \\
\hline \hline$O^{+++} / D^{+}$ & $.3 \mu \mathrm{s}$ & $1 \mu \mathrm{s}$ & $14 \mu \mathrm{s}$ \\
\hline$O^{+++} / e^{-}$ & $20 \mu \mathrm{s}$ & $20 \mu \mathrm{s}$ & $20 \mu \mathrm{s}$ \\
\hline$D^{+} / e^{-}$ & $20 \mu \mathrm{s}$ & $20 \mu \mathrm{s}$ & $20 \mu \mathrm{s}$ \\
\hline
\end{tabular}

Table 6.1: Thermal equilibration times $\left(1 / \nu^{\alpha / \beta}\right)$ between electrons, $D$ and OIV for different values of $T_{\alpha}$, with $T_{\beta}=10 \mathrm{eV}, n_{e}=n_{d}=10^{14} \mathrm{~cm}^{-3}$

The electron temperature was kept constant at $15 \mathrm{eV}$. The scale length $L$ was varied until the simulation matched the initial observed temperature. A best fit was found to be for $L=10 \mathrm{~cm}$. This is reasonable for a typical scale length since the distance between two adjacent electrodes is about $8 \mathrm{~cm}$. The $\mathbf{J} \times \mathbf{B}$ term is turned off after $20 \mu \mathrm{s}$, in order to get agreement with the ol merved data. This could indicate that the $\mathbf{J} \times \mathbf{B}$ force is balanced by $\nabla p$ after the transfer of mass motion into thermal energy. The density was adjusted to match the observed decay in temperature. The best value found was $10^{14} \mathrm{~cm}^{-3}$. Experimentally the average electron density near the center is measured to increase from 0 to $5 \times 10^{14} \mathrm{~cm}^{-3}$ at $\mathrm{t}=100 \mu \mathrm{s}$ as the gas becomes fully ionized and compressed. However, from the presence at early times of OIV and OV, the plasma appears to be fully ionized within few microseconds where the current density peaks. Densities of at least $2 \times 10^{14} \mathrm{~cm}^{-3}$ are expected from the injected neutral gas density. The lower density than expected obtained from the simulation would be consistent with a temperature $T_{i}+T_{e}$ within the filaments higher than the average temperature $\overline{T_{i}}+\overline{T_{e}}$.

Figure 6.5 shows the results of the code for initial values of $T_{D}$ and $T_{O}$ of $1 \mathrm{eV}$. The interpretation is that for low enough initial ion temperatures, 

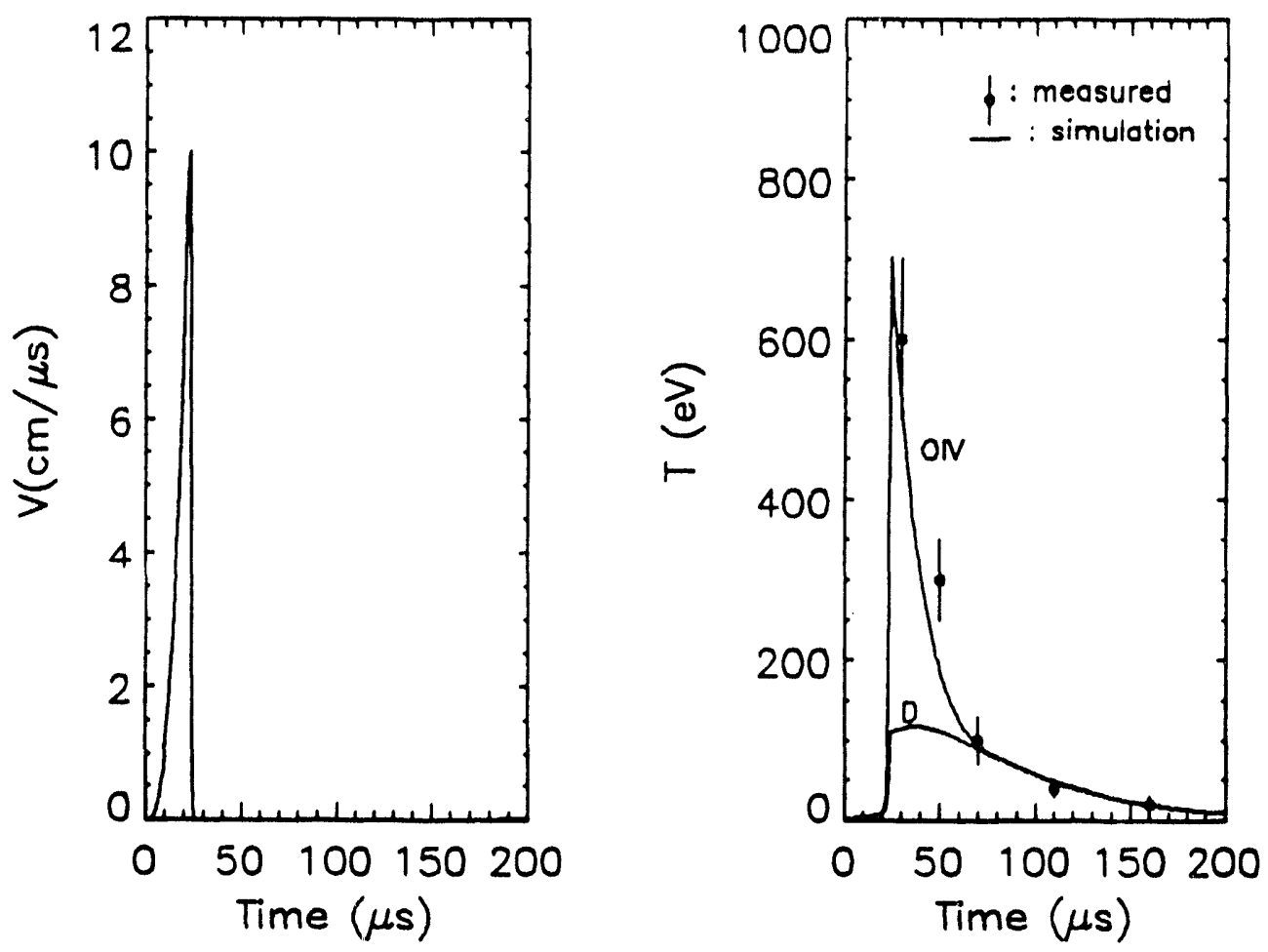

Figure 6.5: Simulation of X-point heating using initial values of $1 \mathrm{eV} T_{D}$ and $T_{O}$ ion temperatures, the electron temperature is fixed at $15 \mathrm{eV}$ and the electron density is fixed at $10^{14} \mathrm{~cm}^{-3}$

viscosity is not initially an important factor and mass flow can increase to an appreciable value. When friction suddenly starts to be important, most of the mass motion energy is transformed into temperature. If the initial ion temperature was higher, then viscosity comes in earlier and no sudden raise of temperature would have occurred since $\mathbf{J} \times \mathbf{B}$ is balancing the friction at all times.

If enough X-points are present in the line of sight, then we have effectively an ensemble of cells in random mass motion. Spectroscopic observations do 
not allow us to differentiate between real temperatures and an ensemble of cells in random mass motion. As mass motion is building up, the observed effective temperature should be increasing until viscosity turns on. At that point we have a real temperature and its rate of change should change suddenly. In the experiment it would then start to decay by equilibrium with the electrons. The data show that initial ion heating occurs within the îrst $30 \mu \mathrm{s}$, in agreement with the simulation.

This scheme could also be valid in the absence of $\mathrm{X}$-points. It would correspond to heating due to the pinching of the plasma. But in that case the $\nabla p$ term in eq. 6.6 could become important sooner than in the $\mathrm{X}$-point case. This simulation demonstrates that $\mathrm{X}$-point heating is a possible mechanism to explain the high ion temperatures observed.

\subsection{Energy balance}

Ohm's law,

$$
-\nabla \phi-\frac{\partial \mathbf{A}_{\mathbf{p}}}{\partial \mathbf{t}}+\mathbf{V} \times \mathbf{B}=\eta \mathbf{J}
$$

gives a power balance equation when multiplied by $J_{p}$, the poloidal current density, and integrated over space:

$$
\Delta \phi_{e l} I_{z}-\frac{\partial}{\partial t} \int_{v o l} \frac{1}{2 \mu_{0}} B_{\phi}^{2} d^{3} x+\int_{v o l}(\mathbf{V} \times \mathbf{B}) \cdot \mathbf{J}_{\mathbf{p}} d^{3} x=\int_{v o l} \eta J_{p}^{2} d^{3} x
$$

where $\phi_{e l}$ is the difference of potential between the electrodes. Because of the nature of the spheromak, some toroidal flux can get converted into poloidal 
flux and vice-versa and this equation alone is inaccurate. One should take into account all the magnetic field components and the effect of the reversal coil itself. Then,

$$
\Delta \phi_{e l} I_{z}+\Delta \phi_{\text {rev }} I_{\text {rev }}-\frac{\partial}{\partial t} \int_{v o l} \frac{1}{2 \mu_{0}} B_{\text {tot }}^{2} d^{3} x+\int_{v o l}(\mathbf{V} \times \mathbf{B}) \cdot \mathbf{J} d^{3} x=\int_{v o l} \eta J^{2} d^{3} x+R_{\text {rev }} I_{\text {rev }}^{2}
$$

The terms represent in order the total power injected through the electrodes and into the reversal coils from the banks, the power going to the magnetic field, the power going to the ions, the power dissipated as Joule heating, and the power dissipated in the reversal coils. If we assume a $15 \mathrm{eV}$ plasma, all the quantities are known or can be measured, except for the ion heating term. The presence of a plasma sheath around the electrodes would dissipate extra power and would leave less power available to the ions.

The simulation suggests that after mass motion energy is transferred into ion temperature, the $(\mathrm{V} \times \mathrm{B}) \cdot \mathrm{J}$ term suddenly goes to zero. This should create a sudden change in the energy input into the plasma. Looking at the power input to the electrodes, (Fig. 6.6), one can see three spikes occuring in the first $20 \mu \mathrm{s}$. The ion heating process could be ocurring three times. It is also possible that the first events correspond to the hydrogen ionization process. Assuming that the spikes are due to power going to the ions, we drew (in dotted line) the power input excluding the power going to the ions. The energy going to the ions is in the order of 500 Joules per spikes. Assuming a density of $2 \times 10^{14} \mathrm{~cm}^{-3}$ and a cylindrical plasma of $40 \mathrm{~cm}$ long and $30 \mathrm{~cm}$ radius, the energy necessary to dissociate and ionize the deutciium atoms is about $50 \mathrm{eV}$. 
If every deuterium ion has a $100 \mathrm{eV}$ temperature, the total kinetic energy is about $360 \mathrm{eV}$. Therefore the energy provided by the spikes in power input is in the right order of magnitude to heat the main species to $100 \mathrm{eV}$. If the origin of the heat is mass flow, then oxygen ions could get $800 \mathrm{eV}$ temperature or more at the $\mathrm{X}$-point locations.

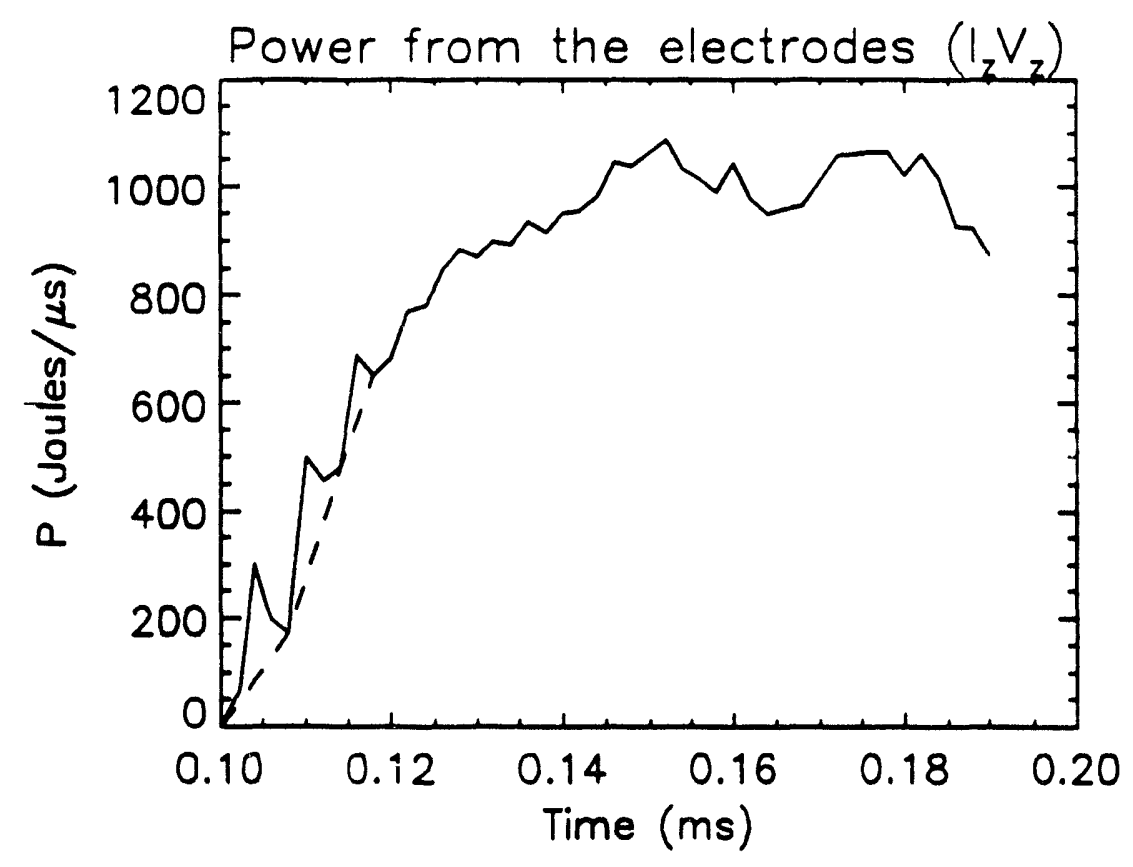

Figure 6.6: Solid line: Total power input through the electrode during formation. Dotted line: Power input excluding the spikes. 


\subsection{Conclusion}

It emerges from the observations and analysis that as a reverse current appears at the center of vessel in the formation phase, on the $r-\phi$ flux surface containing a null toroidal field, a tearing mode instability appears. It is not a detrimental instability as the reverse current seems to stabilize it and it ceases to exist at the same time as this reverse current. $n=3,2$ and finally an $n=1$ modes are observed.

A few microseconds after the discharge is triggered, the plasma is described by MHD equations. The non-uniform fields resulting from the instability could create accelerating and converging flow velocities. The viscosity has a nonlinear dependence on ion temperature and, as it suddenly becomes important, it transforms all the directed energy into thermal energy. The plasma is then collisional. All ions acquire the same directed velocity and therefore their final thermal energy is proportional to the ion mass. In 1 ss than 30 microseconds a $15 \mathrm{eV}$ electron temperature is obtained in the hotter part of the plasma and is maintained at such value due to the radiation barrier. It allows us to observe OIV ions in the path of the $I_{z}$ with thermal energies as high as 600 $\mathrm{eV}$. Deuterium is expected to gain about $80 \mathrm{eV}$. The ion temperature of each species then decays due to equilibration with mainly the electrons.

There is no evidence of anomalous ion heating during the decay phase of MS. Based on other experiments $[6,7]$, this is when ion heating due to helicity transport of fluctuations was expected. We do not know the effect of helicity transport during formation in MS. Since fluctuations usually accompany such 
transport $[44,46]$, measurements of magnetic fluctuations during the formation might determine if helicity transport is an important effect.

It would be interesting as a future experiment to fire the machine with fewer electrodes (4 or 8 instead of 16) as it would increase the typical scale length of ihe plasma. This lowers the viscous friction, gives more time for the velocity to build up and as a consequence could produce more ion heating. It should be noted that viscous heating is the product of an increasing (or decreasing) nonuniform field and and is not expected in a steady state experiment as opposed to helicity transport heating. Also, if the figure-eight coils could be biased at different voltages in order to control the reversed current, one could then see the effect of this current on the ion heating, the impurity content, the plasma lifetime. 
Appendix A

High voltage pulse circuits. 

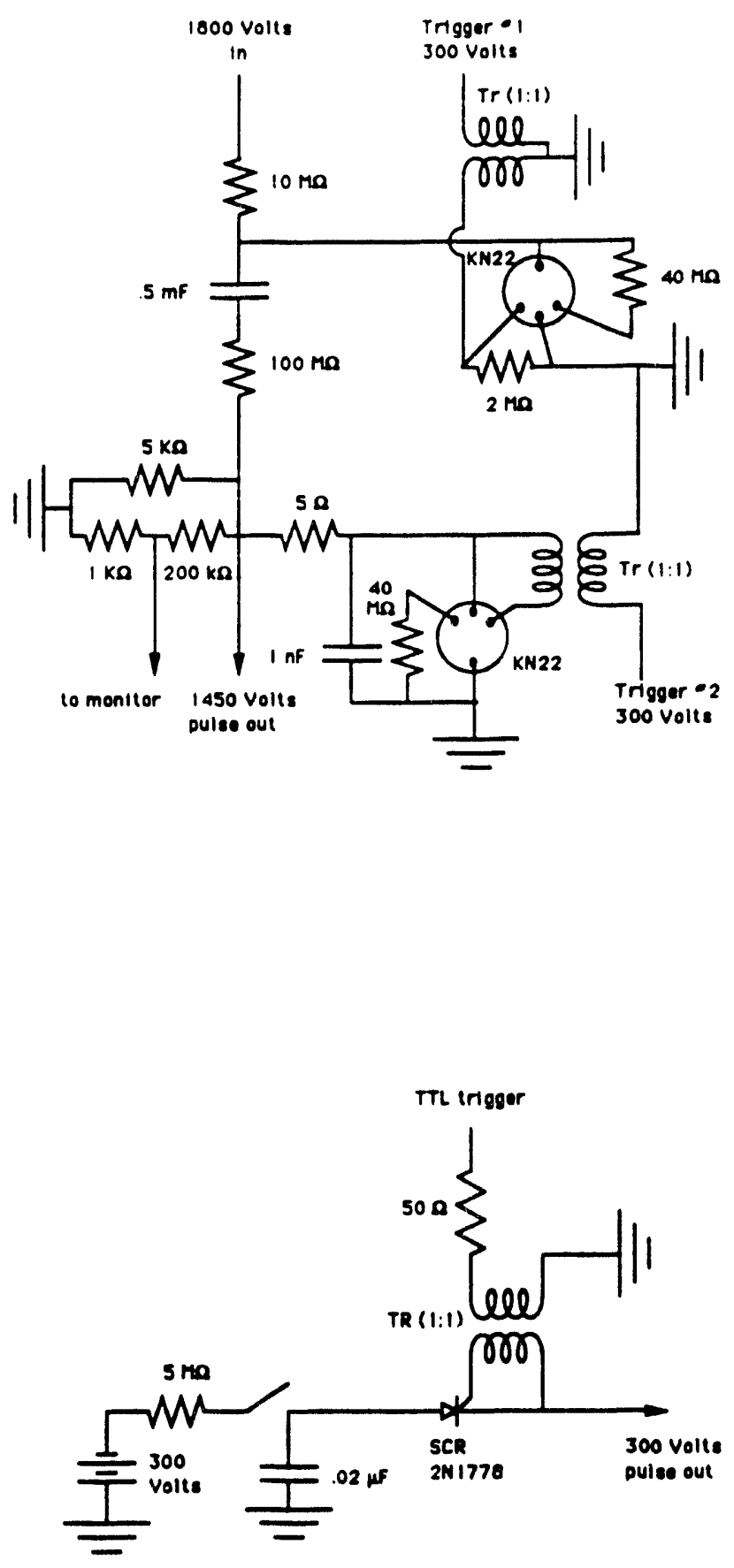

Figure A.1: High voltage circuits used to gate the OMA. Top: Fast switching pulse circuit. Bottom: 300 Volt pulse circuit 


\section{Appendix B}

\section{Ion heating from fluctuations}

This discussion is based on a paper written by A.H. Boozer [47]. Let us consider the modified Ohm's law

$$
\mathbf{E}+\mathbf{V} \times \mathbf{B}=\eta \mathbf{J}-\mathbf{R}_{\mathbf{f}}
$$

where, E, V, B, J are mean field vectors [53] and $\mathbf{R}_{\mathbf{f}}$ is the electric field created by the fluctuations. If we take the scalar product of equation B.1 with B and integrate over space we recover the global helicity balance equation (eq. 6.3), where the transport of helicity is now given by,

$$
\int_{\nu} \mathbf{R}_{\mathbf{f}} \cdot \mathbf{B} d^{3} x
$$


Because helicity is conserved during transport by fluctuations [46], equation B. 2 should be reducible to a surface integral, which corresponds to helicity flow across the boundary without dissipation. We want

$$
\int_{v} \mathbf{R}_{\mathbf{f}} \cdot \mathbf{B} d^{3} x=\int_{\mathbf{s}} \mathbf{h} \cdot \mathbf{d s}=\int_{v} \nabla \cdot \mathbf{h} d^{3} x
$$

Then,

$$
\mathbf{R}_{\mathbf{f}} \cdot \mathbf{B}=\nabla \cdot \mathbf{h}
$$

Boozer shows that for a force-free mean field. $\mathbf{R}_{\boldsymbol{f}}$ must be of the form.

$$
\mathbf{R}_{\mathbf{f}}=\frac{\mathbf{B} \nabla \cdot \mathbf{h}}{B^{2}}
$$

where $\mathbf{h}$ is the flow of helicity carried by the fluctuations.

Then, from 6.4 , the transport of energy due to fluctuations is given by,

$$
\begin{aligned}
\int_{v} \mathbf{R}_{\mathbf{f}} \cdot \mathbf{J}_{\mathbf{0}} d^{3} x & =\int_{v} \frac{\mathbf{B}_{\mathbf{0}} \cdot \mathbf{J}_{0}}{\mathbf{B}^{2}} \nabla \cdot \mathbf{h} d^{3} x \\
& =\int_{v} \mu \nabla \cdot \mathbf{h} d^{3} x \\
& =\int_{v} \nabla \cdot \mu \mathbf{h}-\int_{v} \nabla \mu \cdot \mathbf{h} d^{3} x \\
& =\int_{\mathbf{v}} \mu \mathbf{h} \cdot \mathbf{d} \mathbf{s}-\int_{v} \nabla \mu \cdot \mathbf{h} d^{3} x
\end{aligned}
$$

The first term represents flow of energy across the boundaries while the second term represents dissipation of energy. The power per unit volume going to the ions is then,

$$
\frac{P_{\text {ion }}}{\text { Vol }}=-\nabla \mu \cdot \mathbf{h}
$$


The most general expression for $h$ is $[47]$,

$$
\mathbf{h}=-\left[\lambda-\frac{\nabla \cdot \mathbf{s}}{|\nabla \mu|^{2}}\right] \nabla \mu+\mathbf{u} \times \nabla \mu,
$$

where $\lambda$ has the form of a viscosity preventing $\mu$ from changing over a spatial scale shorter than

$$
\delta^{2}=\frac{\lambda}{\eta / B^{2}}
$$

The parameters $\lambda, \mathbf{u}$ and $\mathbf{s}$ are functions of position.

Boozer shows that the only relevant part for energy dissipation in a forcefree plasma is,

$$
\mathbf{h}=-\lambda \nabla \mu
$$

The other terms contribute only to a surface integral and give energy transport across the boundaries. Finally, the energy dissipated is simply

$$
P_{\text {ion }}=\int \lambda(\nabla \mu)^{2} d^{3} x
$$




\section{Bibliography}

[1] I.E. Tamm and A.D. Saharov, Plasma Physics and the Problem Controlled Thermonuclear Fusion, vol. 1, M.A. Leontovich, ed., Pergammon,NewYork (1961), p. 1.

[2] L. Spitzer Jr, report WASH-115, US Atomic Energy Commision (1952), p. 12.

[3] International Fusion Research Council, Nucl. Fus. 30 (1990) 1643.

[4] D.M. Meade and the TFTR group, Recent TFTR Results, $13^{\text {th }}$ International conference on Plasma Physics and Controlled Nuclear Fusion Research, Washington DC (1990).

[5] P. Rebut and JET team, Recent JET Results and Future Prospects, in $13^{\text {th }}$ International conference on Plasma Physics and Controlled Nuclear Fusion Research, Washington DC (1990).

[6] R.M. Mayo, C.K. Choi, F.M. Levington, A.C. Janos, and M. Yamada, Phys. Fluids B 2 (1990) 115.

(7) J.C. Fernández, C.W. Barnes, T.R. Jarboe, I. Henins, H.W. Hoida, and B.L. Wright, Nucl. Fusion 28 (1988) 1555.

[8] R.B. Howell and Y. Nagayama, Phys. Fluids 28 (1985) 743.

[9] P.G. Carolan, A.R. Field, A. Lazaros, M.G. Rusbridge, H.Y.W. Tsui, and M.K. Bevir, in Controlled Fusion and Plasma Physics (Proc. 14 ${ }^{\text {th }}$ Eur. Conf. Madrid, 1987), Vol 11D, Part II, European Physical Society (1987) 469.

[10] T. Fujita, K Saito, J. Matsui, Y. Kamada, H. Morimoto, Z. Yoshida, and N. Inoue, Nucl. Fus. 31 (1991) 3.

[11] S.C. Jardin and U. Christensen, Nucl. Fus. 21 (1981) 1665.

[12] J.B. Taylor, Rev. Mod. Phys., 58 (1986) 741. 
[13] G.C. Goldenbaum. J.H. Irby, I.P. Chong, and G.IV. Hart Phys. Rev. Lett. $44(1980) 393$.

[14] M. Yamada. H.P. Furth. W. Hsu. A. Janos. S. Jardin. M. Okabayashi. J. Sinnis. T.H. Stix. and K. lamazaki. Phys. Rev. Lett. 46 (1081) 188.

[15] IV.C. Turner. E.H.A. Granneman. C.IV. Hartman. D.S. Prono. J. Taska, and A.C. Smith, J. Applied Phys. 52 (1981) 175.

[16] T.R. Jarboe, Cris W. Barnes, D.A. Platts, and B.L. Wright, CPPCF 9 (1985) 161.

[17] W.C. Turner. G.C. Goldenbaum, E.H.A. Granneman. J.H. Hammer, C.W. Hartman, D.S. Prono, and J. Taska. Phys. Fluid 26 (1083) 1965.

[18] G.W. Hart, Ph.D. thesis. University of Maryland (1983).

[19] T.A. Peyser, Ph.D. thesis, Lniversity of Maryland (1987).

[20] B. Barrow, Ph.D. thesis, University of Maryland (1991).

[21] F.K. Hwang, Ph.D. thesis, University of Maryland (1991).

[22] A.B. Filuk, Ph.D. thesis, University of Maryland (1991).

[23] C. Chin-Fatt, A.W. DeSilva, G.C. Goldenbaum, R.A. Hess, C. Côté, A.B. Filuk, J.-L. Gauvreau, F.K. Hwang, and L. Kanaganayagasingam, Bull. Am. Phys. Soc. 34 (1989) 2052.

[24] W. Baade, Bull. of the Astron. Institutes of the Netherlands 12 (1956) 12.

[25] L. Woltjer, Bull. of the Astron. Institutes of the Netherlands 14 (1958) 39.

[26] H.A.B. Bodin and A.A. Newton, Nucl. Fus. 20 (1980) 1255.

[27] J.B. Taylor, Phys. Rev Lett. 33 (1974) 1139.

[28] M.N. Bussac, H.P. Furth,M Okabayashi, M.N. Rosenbluth, A.M.N. Todd, Plasma Physics and Controlled Nuclear Fusion Research 3 (1978) 249.

[29] H.J. Kunze, in Plasma Diagnostics, ed. W. Lochte-Holtgreven (north Holland, Amsterdam, 1968).

[30] C.J. Buchenauer and A.R. Jacobson Rev. Scient. Instr. 48 (1977) 769.

[31] J.D. Jackson, in Classical Electrodynamics, eds. J. Wiley \& Sons (1975). 
[32] R.D. Cowan, in The Theory of Atomic Structure and Spectra (University of California Press. Berkeley, 1981).

[33] H.R. Griem, in Plasma Spectroscopy (McGraw-Hill. New-York, 1964).

[34] H.R. Griem, in Spectral Line Broadening by Plasmas (Academic Press, New York, 1974).

[35] T. Peyser and G.C Goldenbaum, Phys. Rev. Lett. 61 (1988) 955.

[36] J.-L. Gauvreau, G.C. Goldenbaum, C. Chin-Fatt, A.W. DeSilva, and R.A Hess, Bull. Am. Phys. Soc. 34 (1989) 2052.

[37] C. Jordan, Mon. Not. R. Astr. Soc. 148 (1970) 17.

[38] B.B. Jones, R. Wilson, in Plasma Physics and Controlled Nuclear Fusion Research (Proc. Int. Conf. Salzburg, 1961), Nucl. Fus. 1962 Supplement, Part 3, IAEA, Vienna (1963) 8 S9.

[39] R.B. Howell and Y. Nagayama, Phys. Fluids 28 (1985) $i+3$.

[40] B. Alper, V. Antoni, M.K. Bevir, et al. in Plasma Physics and Controlled Nuclear Fusion Research 1986 (Proc. $11^{\text {th }}$ Int. Conf. Kyoto, 1986), Vol. 2, IAEA, Vienna (1987) 399.

[41] T.R. Jarboe and B. Alper, Phys. Fluids 30 (1987) 1177.

[42] J.C. Fernández, T.R. Jarboe, S.O. Knox, I. Hennins, and G.J. Marklin, Nucl. Fus. 30 (1990) 67.

[43] F.J. Wysocki, J.C. Fernández, I. Hennins, T.R. Jarboe, and G.J. Marklin, Phys. Rev Lett. 65 (1990) 40.

[44] H.A.B. Bodin, Nucl. Fus. 30 (1990) 1717.

[45] B. Alper, H.A.B. Bodin, C.A. Bunting, et al., Plasma Phys. Controll. Fusion 30 (1988) 843.

[46] H.Y.W. Tsui, Nucl. Fus. 28 (1988) 1543.

[47] A.H. Boozer, J. Plasma Phys. 35 (1986) 133.

[48] B.B. Kadomtsev, Plasma Turbulence (Academic, New York, 1965).

[49] E. Hameiri and A. Bhattacharjee, Phys. Fluids 30 (1987).

[50] P.N. Guzdar, J.M. Finn, K.Q. Whang, and A. Bondesonenl, Phys. Fluids 28 (1985) 3154.

[51] J.M.Finn, P.N. Guzdar, Phys. Fluids B 3 (1990) 1041. 
[52] S.I. Braginskii. Rev Plasma Phys. Vol. 1. (consultants Bureau. New York. 1965) 205.

[53] H.K. Moffat, in Magnetic Field Generation in Electrically Conducting Fluids (Cambridge). 

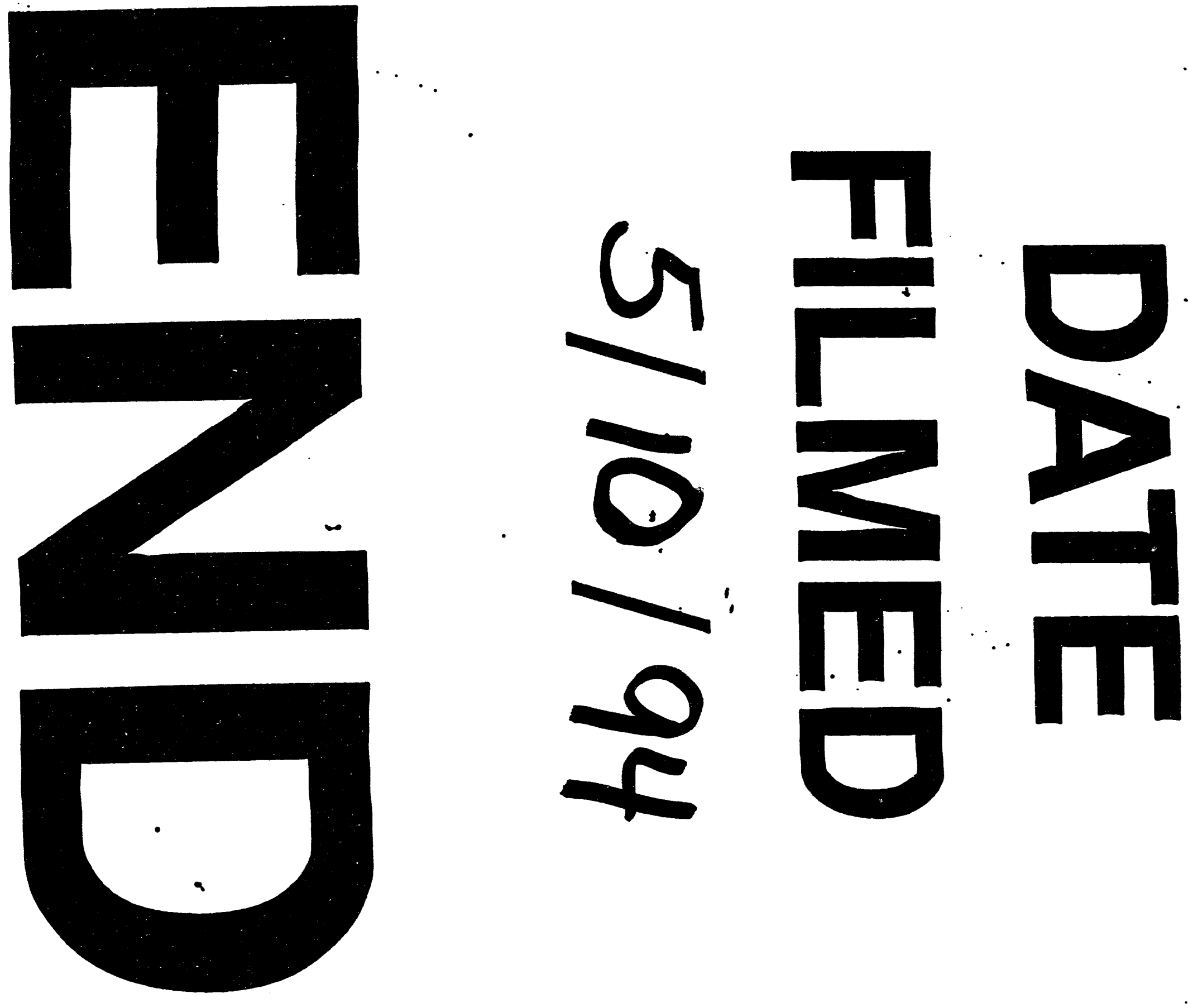